\title{
In Judge Lecompte's Court
}

\author{
M.H. Hoeflich*
}

Today, few remember Judge Samuel Dexter Lecompte except as a committed partisan of the proslavery party in territorial Kansas who used his office to further his party's political goals. But, in fact, Judge Lecompte, the first chief justice of the Kansas Territorial Supreme Court, was a sophisticated jurist instrumental in creating the Kansas legal system. His court was the first outpost of justice in a frontier settlement beset by sectarian strife. The time has clearly come for a reconsideration of the man and the legal structures he helped create.

The twenty-seventh section of the Kansas-Nebraska Act, passed on 30 May 1854, specified that the new Kansas Territory have a "supreme court, district courts, probate courts, and justices of the peace."1 The supreme court was to be comprised of a chief justice and two associate justices. These three justices were each to preside over and reside in one of the three judicial districts into which the territory was divided. Each district court was to have both common law and chancery powers, and each court was authorized to appoint a clerk. $^{2}$ Appeals from the decisions of the Territorial Supreme Court were to be made to the U.S. Supreme Court. ${ }^{3}$

President Franklin Pierce appointed Samuel Dexter Lecompte of Maryland to be chief justice and Sanders W. Johnson of Ohio and Rush Elmore of Alabama to be associate justices of the court. ${ }^{4}$ Soon after taking up his commission on 11 June 1854, Judge Lecompte left Maryland for Kansas. ${ }^{5}$ His two associate justices joined him there. The Territorial Supreme Court held its first session at the Shawnee Manual Labor School on 30 July 1855. In addition to swearing in Marcus Parrot

\footnotetext{
* John H. and John M. Kane Distinguished Professor of Law, University of Kansas School of Law.

1. Kansas-Nebraska Act, ch. 59, § 27, 10 Stat. 277, 286 (1854).

2. Id.

3. Id. at 287 .

4. James McCahon, Reports of Cases Determined in the Supreme Court of the TERRITORY OF KANSAS, at vi (Chicago, Callaghan \& Cockcroft 1870).

5. Id.
} 
as the court's reporter, the justices heard a petition from the territorial district attorney asking them to rule on the validity of the laws passed by the temporary legislature. ${ }^{6}$ The judges fell into controversy over whether to do so. This inauspicious beginning was prophetic. Over the next several years, the court was mired in controversy over the appointments

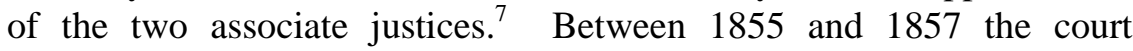
functioned with only two justices, Lecompte and Cato, because Jeremiah Burell fell ill, returned to Pennsylvania and died. ${ }^{8}$ Finally, at the beginning of the June term 1859, John Pettit replaced Justice Lecompte as chief justice. ' Throughout the period of Lecompte's chief justiceship, the court was deeply involved in the widespread political battles across the territory. ${ }^{10}$ Lecompte, himself, was perceived to be openly biased towards the proslavery party, many of whom came from Missouri, and was a primary target of the antislavery forces' rhetoric.

In addition to his duties as chief justice of the Territorial Supreme Court, Lecompte was appointed district judge of the First Judicial District of Kansas. ${ }^{11}$ That district consisted of all the counties north of the Kaw and south of the Nebraska border. These were Doniphan, Atchison, Leavenworth, Jefferson, Calhoun Counties. Soon, Douglas County was added to the district. ${ }^{12}$ Judge Lecompte rode circuit through his district: court sessions were to be held in Leavenworth on the third Monday of September, Atchison on the fourth Monday of September, Doniphan on the first Tuesday in October, Jefferson on the second Monday, Calhoun on the third Monday, and Douglas on the fourth Monday. ${ }^{13}$ A similar circuit was required in the spring and summer.

When one considers both the difficulty of travel among these counties under normal conditions and the even greater difficulties caused by the roving bands of Free-State and proslavery marauders, it is quite remarkable that Lecompte was able to hold even a small fraction of the

6. Id. at vii-viii.

7. Id. at viii-x. Four new justices, Jeremiah Burrell, Sterling G. Cato, Thomas Cunningham, and Joseph Williams all presented presidential commissions between 1855 and 1857. MICHAEL H. Hoeflich, Justice on the Prairie: 150 Years of the Federal District Court in Kansas 4 (2011).

8. HoEfLICH, supra note 7, at 4 .

9. MCCAHON, supra note 4 , at $\mathrm{x}$.

10. HOEFLICH, supra note 7, at 3-7.

11. Letter from Justice Lecompte to Governor Geary (Oct. 6, 1856), in 4 TransaCtions OF the Kansas State HistoriCal Society, at 603 (Topeka, Kansas Publishing House, 1890).

12. Id. at 604

13. Id. 
sessions required by the state legislature. In fact, Lecompte came under attack for dereliction of duty. In letters to Governor Geary ${ }^{14}$ and Congressman James A. Stewart of Maryland, ${ }^{15}$ Lecompte argued that he had not been absent from his district except for several nights of travel. In his letter to Governor Geary, he stated that he had held all required sessions in 1855 and the first term of 1856 except for one session in Doniphan County, which he could not reach because of "failure of the boats" to St. Joseph from where he would have embarked for Doniphan. ${ }^{16}$ During the next terms, Lecompte again met all his circuit obligations except for sessions in Jefferson and Calhoun Counties because of "commotions" in these counties and his belief that he would have been unable to bring juries or witnesses into court. ${ }^{17}$

If one accepts Judge Lecompte's accounts of his circuit ridings, then it would appear that the majority of the legislatively required court sessions were held with Judge Lecompte presiding even during the worst days of what has come to be called "Bleeding Kansas." 18

\section{PROCEDURE IN THE FIRST DISTRICT COURT}

The records of the district courts now in the Territorial Kansas Collection at the Kansas State Historical Archives provide some information about the lawyers and litigants who appeared in these courts, the matters they litigated, and the outcomes of the cases. ${ }^{19}$ Further, the Kansas State Library holds the unique printed copy of the Rules of the First District Court adopted in August 1856. ${ }^{20}$ The Rules run to fifteen printed pages and are highly sophisticated, certainly not what one might expect of a frontier court.

\footnotetext{
14. Id.

15. Letter from Judge Lecompte to Hon. James. A. Stewart of Maryland (Aug. 1, 1856) (on file with the KSHS), available at http://www.civilwaronthewesternborder.org/content/letter-judgelecompte.

16. Letter from Justice Lecompte to Governor Geary, supra note 11, at 604.

17. Id. at 605 .

18. There is a large literature on the history of the conflict in Territorial Kansas. Among the more recent and best secondary accounts of this period is NICOLE ETCHISON, BLEEDING KANSAS: CONTESTED LIBERTY IN THE CIVIL WAR ERA (2004).

19. These materials are indexed online in Territorial KANSAS OnLine, http://www.territorialkansasonline.org/, a site maintained by the KSHS.

20. Samuel D. Lecompte, Rules of the First District Court, of the United States FOR THE First Judicial District of THE TERRITORY OF KANSAS (St. Joseph, Millan \& Postgate 1856). It is interesting to note that the rules were printed in St. Joseph, Missouri in September 1856. Is it possible that Lecompte was unable to find a Kansas printer because of his perceived bias for the (strongly Missouri) proslavery faction?
} 
The Rules of the First District Court are divided into eight sections: "Rules of Government," "For the Regulation of Pleading," "Regulating Appeals," "As to the Rules Themselves," "Officers of the Court," "Order of Business," "Rules in Chancery," and "Order of Business." 21 The officers of the court were comprised of the marshal and his deputies, bailiffs, and a clerk of the court. ${ }^{22}$ The marshal and his staff were authorized to enforce orders of the court, serve warrants, and summon jurors. The clerk of the court was authorized to issue subpoenas and

. . . keep an alphabetical list of every judgment in the names of the plaintiff and defendant, together with the time when rendered, and a specification of the form of action, whether ejectment, dower, debt, case, covenant, trespass, or replevin.

The rules for the order of business of the court are quite detailed. The court was to be called at 10:00 a.m. with a proclamation of the court crier. $^{24}$ On the first day of court, juries and a grand jury were to be charged. ${ }^{25}$ Once this was accomplished, the dockets were to be called: first the appearance docket, followed by the trial and subpoena dockets, and then the appellate docket. ${ }^{26}$ Generally, non-jury trials were not to be heard until all the jury trials had been finished. ${ }^{27}$ In addition, the Rules specify that criminal cases should "take precedence" over civil cases and "that as the Kansas bill requires csues [sic] arising under Statutes of Congress, shall be entitled to precedence during the first six days of the term." 28

One of the more interesting aspects of the Rules of the First District Court, particularly in light of Judge Lecompte's account of the difficulties of holding court in some instances, are the statements that prescribe for the Rules to be followed with flexibility:

21. Id. It is unclear why there are two sections that address the "Order of Business" within the publication.

22. Id. at 10 .

23. Id. Many of these are now to be found in manuscript form at the KSHS in its Territorial Kansas Collection.

24. Id. at 15.

25. Id. at 10 .

26. There is a seeming conflict in the printed RULES OF THE FIRST DISTRICT COURT on whether trials or appeals would be heard first. The RULES, at 10, suggest that the order would be trials and then appeals. The RULES, at 15, specify the reverse order. Interestingly, the RULES, at 15 , specify that appeals may be heard by a jury, quite unlike modern procedure.

27. Id. at 3 .

28. Id. at 11,15 . The reference is to the Kansas-Nebraska Act of 1854 . 
These rules being regarded as creatures of the Court, and intended not to oppress but relieve suitors, their Counsel, and the Court of embarrassment, are to be considered subject to modification, enlargement or repeal, the more especially in view of the novelty of our situation as a people, and the necessary uncertainty their operation in the new cases in which they are applied. ${ }^{29}$

And:

The Court will expect its rules to be observed, and will be rigid in the enforcement of them, but will, nevertheless, be disposed so liberally as to construe them as not to hinder the advancement of justice, or to expose any one to an unreasonable hardship. ${ }^{30}$

From this statement, we may deduce that Judge Lecompte realized that the detailed rules of the court, which he had adopted in his district, might well prove difficult to enforce strictly at all times. One can imagine quite easily that travel conditions in a frontier community might make it impossible for lawyers and litigants to appear precisely at 10:00 a.m. every day! ${ }^{31}$

Another of the rules also speaks to the difficulty of ensuring attendance by all the necessary parties:

The non-attendance of Counsel will not be considered good reason for the delay or postponement of a trial, unless occasioned by sickness, or unavoidable impediment, but all causes marked for trial must be tried as they are called unless continued upon legal grounds. ${ }^{32}$

Several other rules demonstrate the difficulties faced by Judge Lecompte and his court staff. One rule prohibited anyone from removing any original papers filed with the court, presumably for fear that theft of

\footnotetext{
29. Id. at 10 .

30. Id. at 9 .

31. An insight into how difficult living and travelling conditions were during the early territorial period may be gained by noting that the first legislature attempted to remove the site of their meeting from Pawnee to the Shawnee Mission Labor School because "at Pawnee there was no place of accommodation, and members had to camp out, sleep in their wagons or tents, and cook their own provisions ....." STATUTES OF THE TERRITORY OF KANSAS 1855, at vii (Shawnee Mission, John T. Brady, 1855). An illustration of a territorial court —or at least an artist's rendering of one in 1876 - may be found on page 33 of REQUISITE LEARNING AND GOOD MORAL Character: A History of THE KANSAS BenCH AND BAR (Robert W. Richmond ed., 1982).

32. LECOMPTE, supra note 20, at 3.
} 
court papers would prevent trials from proceeding. ${ }^{33}$ One must remember that the court did not generally have a secure, dedicated courthouse in which to sit during this early period. Theft of vital documents would have been relatively easy to accomplish. Another rule provided that litigants could choose to forego a trial and choose, instead, to have their dispute heard by arbitrators:

Any action constituted in this Court may with the consent of the parties be referred to the arbitrament and award of arbitrators chosen by the parties with power to choose an umpire ....

It is easy to understand why parties might choose to avoid trial and go to arbitration. Like today, the costs would likely be lower and the proceedings faster. In addition, the applicable law in the territory would have been either difficult to discover because of the lack of law books or unclear because of the unique situation in the new territory. ${ }^{35}$

\section{THE BUSINESS OF THE FIRST DISTRICT COURT}

In spite of civil unrest and the difficulties of travel, Judge Lecompte's court was quite busy. In his letter to Governor Geary, Judge Lecompte relayed that there were sixteen indictments during the April 1855 term for Leavenworth. ${ }^{36}$ During the October term, there were twenty-one indictments and twenty-two civil suits filed. He attributed the low number of civil suits to the absence of territorial statutes, which had not yet been published. ${ }^{37}$ In November (an adjourned term from October) there were fourteen indictments. ${ }^{38}$ During March term 1856, there were seventeen appeals, twenty-five original civil suits, fifteen attachments and habeas corpus cases, and seventy-nine indictments. ${ }^{39}$

33. Id. at 4. Another indication of the difficulty in which the court found itself regarding documents is demonstrated by an order of the Territorial Supreme Court issued during December term 1858: "It is ordered by the Court that the Clerk furnish copies of transcripts in each case to each judge thereof."

34. LECOMPTE, supra note 20, at 6. Examples of cases that went to arbitration are to be found in the KSHS archives.

35. On the paucity of law books see Robert A. Mead \& M.H. Hoeflich, Lawyers and Law Books in Nineteenth-Century Kansas, 50 U. KAN. L. REV. 1051 (2002).

36. Letter from Justice Lecompte to Governor Geary, supra note 11, at 605.

37. Id. The first territorial statutes were published at the Shawnee Mission Labor School with a preface dated 1 November 1855. STATUTES OF THE TERRITORY OF KANSAS 1855, supra note 31, at vii.

38. Letter from Justice Lecompte to Governor Geary, supra note 11, at 605.

39. Id. 
During August term 1856, there were twenty-eight appeals, forty-three original suits, and twenty-nine trials.

What kinds of cases were heard during this period in the First Judicial District and Territorial Supreme Court? The majority of the criminal cases were for relatively commonplace crimes: murder, theft, selling liquor without a license, selling liquor to Native Americans. The late Judge George Templar noted that what was missing from the docket, for the most part, were criminal cases brought against the proslavery partisans wandering through the territory and committing acts of violence. $^{40}$ The criminal indictments relating directly to the political struggles appear to have been aimed at members of the Free-State party. ${ }^{41}$ Judge Lecompte, on the other hand, vehemently denied that he showed any political bias in his courtroom. ${ }^{42}$

The late Professor Paul Wilson characterized the nature of litigation in frontier Kansas as "simple, if not crude, and in most places a comprehensive knowledge of the law or special technical training in legal skills was not required and was likely to be of little use to the average lawyer." 43 The nature of the cases heard in Judge Lecompte's court suggests that Professor Wilson was right on the mark. A manuscript list maintained by the clerk during the December 1856 term gives us a sense of the types of cases heard by the Territorial Supreme Court. ${ }^{44}$ Thirty-one cases were heard during this term. Among these were trials for mandamus, selling liquor without a license, shooting with the intent to kill, murder, debt, issuance of an injunction, trespass, attachment, assumpsit, and unlawful detainer. ${ }^{45}$ Of these, six involved an action in debt. Six involved assumpsit. Two involved attachment and two involved unlawful detainer. In addition, five involved a bill in chancery. ${ }^{46}$

An additional source useful for reconstructing the business of the

40. See George Templar, The Federal Judiciary of Kansas, in ReQUISITE LEARNING AND GOOD MORAL CHARACTER, supra note 31, at 62.

41. Id. at 61.

42. Letter from Judge Lecompte to Hon. James. A. Stewart of Maryland, supra note 15; Letter from Justice Lecompte to Governor Geary, supra note 11, at 603 ("If it [the charge of political bias] means more than the fact, and to intimate that this 'party bias' has affected the integrity of my official action, in any solitary case, I have but to say that it is false- basely false.").

43. Paul E. Wilson, The Early Years: The Bench and Bar Before 1882, in REQUISITE LEARNING AND GOOD MORAL CHARACTER, supra note 31, at 37.

44. Kansas Supreme Court, Trial Docket A (unpublished manuscript) (on file with the KSHS).

45. Id.

46. Id. 
Kansas territorial courts are the manuscript case files now at the Kansas State Historical Society. ${ }^{47}$ The contents of these case files vary from complete records to single documents, but virtually all contain the names of the litigants and the subject of the litigation. A small sample of these criminal case records filed in Judge Lecompte's First Judicial District shows the accuracy of Professor Wilson's statement:

April 1856: KT v. Biglow: selling liquor without a license ${ }^{48}$

Sept. 1856: KT v. Alderman: $\operatorname{arson}^{49}$

Sept. 1856: U.S. v. Chapman: murder $^{50}$

Sept. 1856: KT v. Cleary: murder $_{52}^{51}$

Sept. 1856: KT v. Davis: larceny ${ }_{53}^{52}$

Oct. 1856: KT v. Crane: robbery ${ }^{53}$

Oct. 1856: U.S. v. Crane: robbing U.S. mail ${ }^{54}$

Oct. 1856: KT v. Cahee: larceny ${ }^{55}$

Oct. 1856: U.S. v. Bruno: selling liquor to Indians ${ }^{56}$

Oct. 1856: KT v. Cutler, Dolman, Lane: assault and robbery ${ }^{57}$

[ ] 1856: KT v. Coleman: horse stealing ${ }^{58}$

Aug. 1857: KT v. Burke: keeping a gaming house ${ }^{59}$

Sept. 1857: KT v. Burke: selling liquor on Sunday ${ }^{60}$

April 1858: KT v. Richard Alous: selling liquor without a license ${ }^{61}$

April 1858: U.S. v. Bowen: Grand Larceny ${ }^{6}$

April 1858: KT v. Conway: selling liquor without a license ${ }^{63}$

Sept. 1858: U.S. v. Buckmaster: taking Indian property $^{64}$

Oct. 1858: KT v. Bainter: robbery of a store ${ }^{65}$

\footnotetext{
47. These are maintained in the Kansas Territorial Records, 1854-1861 Collection. An index to these records may be found online at http://www.kshs.org/research/collections/documents/govtrecords/KSTerritorialRecords.pdf. Sub group 5, consisting of twenty boxes of papers and four bound manuscript volumes, comprises the case records of the U.S. District Court for Kansas from 1854-1860.

48. KSHS 27-02-05-07 Folder 08.

49. KSHS 27-02-05-06 Folder 08.

50. KSHS 27-02-06-01 Folder 17.

51. KSHS 27-02-06-01 Folder 41.

52. KSHS 27-02-06-03 Folder 11.

53. KSHS 27-02-06-02 Folder 28.

54. KSHS 27-02-06-02 Folder 29.

55. KSHS 27-02-06-02 Folder 04.

56. KSHS 27-02-05-07 Folder 53.

57. KSHS 27-02-06-02 Folder 56.

58. KSHS 27-02-06-02 Folder 09.

59. KSHS 27-02-05-07 Folder 60.

60. KSHS 27-02-05-07 Folder 61.

61. KSHS 27-02-05-06 Folder 14.

62. KSHS 27-02-05-07 Folder 21.

63. KSHS 27-02-06-02 Folder 16.

64. KSHS 27-02-05-07 Folder 54.
} 
Jan. 1859: U.S. v. Berry: assault with intent to kill ${ }^{66}$

Jan. 1859: U.S. v. Bertrand: selling liquor to Indians ${ }^{67}$

These records indicate that the First Judicial District was, indeed, a violent place, but the violence was clearly not limited to the partisan struggle between Free-State and proslavery forces. The accounts of frequent murders in territorial newspapers suggest that murder was not solely a political crime. For instance, the Herald of Freedom, a Lawrence newspaper, reported six murders or possible murders between March 1855 and December $1856 .^{68}$ Indeed, the case records indicate that common theft and illegal sales of liquor were also a great problem for the First Judicial District. As to civil suits, it seems that the court did perform an important function and handled routine civil matters as well as it could under the circumstances. One thing is clear from the records: the First Judicial District under Judge Lecompte was not idle.

The court performed two other important functions. First, the court heard petitions for citizenship from foreign nationals. The court records, particularly for the latter part of the 1850 s, make it clear that this was an important and frequent function. Undoubtedly, the efforts of both Eastern abolitionists and Southern proslavery parties to send supporters into the territory meant that many foreign-born men and women would have reached the territory and sought citizenship and the right to vote.

The second additional function performed by the court was the admission of attorneys. This was not a simple task. The courts of the Kansas Territory attracted a large number of would-be lawyers. Many lawyers, finding few opportunities in the crowded East, followed Horace Greeley's advice to "go West young man." 69 Many of these lawyers settled in the major cities-Lawrence, Lecompton, and Leavenworth. A number maintained offices across the border in Missouri. ${ }^{70}$ Virtually all rode circuit and practiced in several courts throughout the district and the territory. At the first sitting of Justice Lecompte's court in April 1855,

\footnotetext{
65. KSHS 27-02-05-06 Folder 28.

66. KSHS 27-02-05-06 Folder 62.

67. KSHS 27-02-05-06 Folder 63.

68. Herald of FreEdom, Mar. 24, 1855; Nov. 24, 1855; Jan. 26, 1856; Apr. 12, 1856; May 3 , 1856. The latter reports the celebrated murder of R.P. Brown.

69. See Michael H. Hoeflich, The Lawyers of Old Lecompton, in TALLGRASS EsSAYS: PAPERS FROM THE SYMPOSIUM IN HONOR OF DR. RAMON POWERS 27, 27-35 (Michael H. Hoeflich, Gayle R. Davis, \& Jim Hoy eds., 2003).

70. Id. at $29-31$.
} 
sixteen lawyers were admitted to the territorial bar. ${ }^{71}$ By the end of the 1858 term, one hundred sixty-two lawyers had been admitted at Leavenworth. $^{72}$ For a sparsely populated territory, this was a surprisingly large number of lawyers. Indeed, in retrospect, there appears to have been a lawyer glut; many of the admitted lawyers were forced to find other occupations in order to earn a living. Many edited newspapers, collected debts for Eastern creditors, and speculated in land.

The territorial statutes imposed few requirements for bar membership. ${ }^{73}$ Chapter 11 of the 1855 Statutes established the standards for legal practice in the territory. In order to practice law, one had to be "a free white male," produce "testimonials of good moral character," and "undergo a strict examination, as to his qualifications, by one of the judges." 74 Each lawyer had to swear an oath of office including a statement that he would "support and sustain the provisions of an act entitled 'an act to organize the territories of Nebraska and Kansas,' and the provisions of an act commonly known as 'the Fugitive Slave Law." "75 Obviously, this requirement to uphold the Fugitive Slave Act would have been an impediment to abolitionist bar applicants. Nevertheless, many members of the bar so swore and still supported the Free-State cause. ${ }^{76}$

Once an applicant was admitted to the bar, his name was listed in the "roll of attorneys" kept by the clerk of the court. ${ }^{77}$ A lawyer was required to "practice to the best of his knowledge and ability.", If an admitted attorney was found guilty of "any felony or infamous crime, or improperly retaining his client's money, or of any mal-practice, deceit or misdemeanor in his professional capacity," he could be "removed or suspended from practice," by the district court, the supreme court, or another court of record. ${ }^{79}$ These ethical rules certainly echo our current

71. Paul E. Wilson, The Early Years: The Bench and Bar Before 1882, in REQUISITE Learning and GoOd Moral Character, supra note 31, at 35. H. Miles MoOre, Early HISTORY OF LEAVENWORTH CITY AND COUNTY 244-331 (1906) lists each lawyer admitted during the territorial period by name in chronological order of admission and provides a brief biography of each.

72. Paul E. Wilson, The Early Years: The Bench and Bar Before 1882, in REQUISITE LEARNING AND GOOD MORAL CHARACTER, supra note 31, at 36.

73. Id.

74. Id.; STATUTES OF THE TERRITORY OF KANSAS 1855, supra note 31, at 132-34.

75. STATUTES OF THE TERRITORY OF KANSAS 1855, supra note 31, at 132.

76. Kansas Supreme Court, Trial Docket A, supra note 44.

77. StatUTES OF THE TERRITORY OF KANSAS 1855, supra note 31, at 132.

78. Id.

79. Id. at $133-34$. 
Rules of Professional Conduct, albeit within a narrower scope.

Although the 1855 Statutes prescribe that bar applicants swear to uphold the Kansas-Nebraska Act and the Fugitive Slave Law, they do not specify other requirements for the admission oath. This seems to have been left to judicial discretion. Thus, it is interesting to note the form of oath required by Justice Lecompte in April 1855 (predating the publication of the 1855 Statutes by several months):

I . . do solemnly promise and swear (or solemnly, sincerely and truly declare and affirm,) that I will well and properly behave and demean myself in the office of Attorney for the First District Court for the First Judicial District of the territory of Kansas, in all things appertaining to the duties of such office, according to the best of my skill and judgment, and that I will support the constitution and laws of the United States and of said territory. I believe in the divinity of the Christian religion. ${ }^{80}$

Two aspects of this oath are particularly notable. First, unlike the oath later required by the 1855 Statutes, the oath administered by Judge Lecompte in April 1855 did not require an applicant to swear to uphold the Fugitive Slave Law. Given Judge Lecompte's open support for the proslavery party in the political arena, the absence of this clause in the oath may well support his statements that he did not let his politics enter the discharge of his judicial duties. Second, the inclusion in the oath that the applicant believes "in the divinity of the Christian religion," raises interesting questions as to Justice Lecompte's views on the doctrine of church-state separation. ${ }^{81}$

\section{JUSTICE LECOMPTE’S LATER LIFE}

Despite the antipathy felt by many of the Free-State partisans and their efforts to remove him, Justice Lecompte managed to complete his four-year term and retired from office on 9 March $1859 .{ }^{82}$ Upon leaving office, he opened a law practice in Leavenworth. ${ }^{83}$ After the Civil War, Lecompte, once a fervent, proslavery Democrat, joined the Republican

\footnotetext{
80. MOORE, supra note 71 , at 244-45.

81. Don Drakeman, Esq. and I are currently working on an article regarding the significance of the inclusion of this sentence in the 1855 oath.

82. Samuel Dexter Lecompte, 1814-1888, TERRITORIAL KANSAS OnLINE, http://www.territorialkansasonline.org/ imlskto/cgi-

bin/index.php?SCREEN=bio_sketches/lecompte_samuel (last visited June 6, 2014).

83. Id.
} 
Party. ${ }^{84} \mathrm{He}$ served as a probate judge in Leavenworth for four years. ${ }^{85}$ In 1867, Lecompte was elected to the legislature from Leavenworth. Interestingly, he was also named the "Poet Laureate" of the Kansas Legislature and, pursuant to this office, he wrote a satirical poem containing comments about each legislator. ${ }^{86}$ In 1874 , he was elected chairman of the First District Republican Congressional Committee. ${ }^{87}$ In 1887, Lecompte moved to Kansas City and died the following year. ${ }^{88}$

Justice Lecompte maintained his innocence against all charges of political bias and malfeasance in office until the day he died. In an 1887 letter to Franklin G. Adams, the Executive Director of the Kansas State Historical Society, Lecompte noted the "insufferable wrongs" and "most injurious falsehoods" that had been leveled against him by so many Kansans. ${ }^{89}$ He complained that his name had become the "synonym of corruption and degradation of power" even though he had "performed every duty ... with an entire freedom from partisanship [sic] or personal prejudice." ${ }^{90}$ It is clear that Justice Lecompte felt that he had been wronged and that he would die unjustly condemned. Whatever the truth of the accusations of partisanship made against him, it must be said that Lecompte's four years as a territorial judge helped establish the rule of law in Kansas.

84. Id. MoORE, supra note 71, at 311, states that Lecompte "was not a brilliant success at the bar" and, incorrectly, that after a few years returned to the East.

85. Samuel Dexter Lecompte, 1814-1888, supra note 82.

86. House Journal of the Legislative AsSembly of the STAte of Kansas 1060-64 (Leavenworth, Clarke, Emery \& Co., 1867).

87. Samuel Dexter Lecompte, 1814-1888, supra note 82.

88. Id.

89. Letter from S.D. Lecompte to F.G. Adams (Mar. 16, 1887) (on file with KSHS in the Lecompte Papers).

90. Id. 
APPENDIX OF TERRITORIAL KANSAS CRIMINAL CASES ${ }^{91}$

\begin{tabular}{|c|c|c|c|c|}
\hline Crime & District & Defendant & Date & $\begin{array}{l}\text { Folder } \\
\text { Reference }\end{array}$ \\
\hline $\begin{array}{l}\text { Aiding and } \\
\text { Abetting a } \\
\text { Fugitive }\end{array}$ & $1 \mathrm{st}$ & Wilson, James & $6 / 28 / 1859$ & $\begin{array}{l}27-02-08-01 \mathrm{~F} . \\
65\end{array}$ \\
\hline $\begin{array}{l}\text { Aiding and } \\
\text { Abetting an } \\
\text { Escapted } \\
\text { Fugitive }\end{array}$ & $1 \mathrm{st}$ & Bailey, David & $6 / 28 / 1859$ & $\begin{array}{l}27-02-05-06 \mathrm{~F} . \\
24\end{array}$ \\
\hline $\begin{array}{l}\text { Aiding and } \\
\text { Abetting an } \\
\text { Escapted } \\
\text { Fugitive }\end{array}$ & $1 \mathrm{st}$ & Boyle, George & $6 / 28 / 1859$ & $\begin{array}{l}27-02-05-07 \mathrm{~F} . \\
24\end{array}$ \\
\hline Arson & $1 \mathrm{st}$ & Alderman, Henry & $8 / 1856$ & $\begin{array}{l}27-02-05-06 \mathrm{~F} . \\
08\end{array}$ \\
\hline Arson & & Hurd, Jacob & $11 / 4 / 1857$ & $\begin{array}{l}27-02-06-06 \mathrm{~F} . \\
38\end{array}$ \\
\hline Arson & 1st, 2nd & $\begin{array}{l}\text { Morell, Allen \& } \\
\text { Palmer, Francis }\end{array}$ & $9 / 1 / 1856$ & $\begin{array}{l}27-02-07-02 \mathrm{~F} . \\
46\end{array}$ \\
\hline Arson & $1 \mathrm{st}$ & $\begin{array}{l}\text { Smith, Andrew } \\
\text { \& William, Jesse } \\
\text { \& Cleary, John }\end{array}$ & $6 / 1 / 1857$ & $\begin{array}{l}27-02-07-05 \mathrm{~F} . \\
50\end{array}$ \\
\hline Arson & 2nd & $\begin{array}{l}\text { Unberger, } \\
\text { George }\end{array}$ & $2 / 11 / 1858$ & $\begin{array}{l}27-02-07-07 \mathrm{~F} . \\
40\end{array}$ \\
\hline Arson & 2nd & $\begin{array}{l}\text { White, Andrew } \\
\& \text { Curtin, Alfred }\end{array}$ & $9 / 15 / 1856$ & $\begin{array}{l}27-02-08-01 \mathrm{~F} . \\
40\end{array}$ \\
\hline Assault & Shawnee Cnty. & Armstrong, A.J. & $1 / 1857$ & $\begin{array}{l}27-02-05-06 \mathrm{~F} . \\
19\end{array}$ \\
\hline Assault & $?$ & $\begin{array}{l}\text { Armstrong, A.J. } \\
\text { \& Bartel, } \\
\text { Fredrick }\end{array}$ & $12 / 1856$ & $\begin{array}{l}27-02-05-06 \mathrm{~F} . \\
18\end{array}$ \\
\hline Assault & $3 \mathrm{rd}$ & Coombs, James & $10 / 22 / 1858$ & $\begin{array}{l}27-02-06-02 \mathrm{~F} . \\
12\end{array}$ \\
\hline Assault & 2nd & $\begin{array}{l}\text { Cottman, } \\
\text { Marshal }\end{array}$ & $5 / 8 / 1856$ & $\begin{array}{l}27-02-06-02 \mathrm{~F} . \\
02\end{array}$ \\
\hline Assault & Douglas Cnty. & Fuller, R. & $9 / 23 / 1858$ & $\begin{array}{l}27-02-06-04 \mathrm{~F} . \\
61\end{array}$ \\
\hline Assault & 2nd & $\begin{array}{l}\text { Knight, Francis } \\
\text { \& Knight, } \\
\text { Wilder }\end{array}$ & $2 / 4 / 1858$ & $\begin{array}{l}27-02-06-07 \mathrm{~F} . \\
58\end{array}$ \\
\hline Assault & $1 \mathrm{st}$ & McAfee, J.B. & 9/19/18577 & $\begin{array}{l}27-02-07-01 \mathrm{~F} . \\
57\end{array}$ \\
\hline Assault & 2nd & McCallister, U.S. & $5 / 1 / 1860$ & $\begin{array}{l}27-02-07-01 \mathrm{~F} . \\
58\end{array}$ \\
\hline Assault & $1 \mathrm{st}$ & McGee, Thomas & $9 / 14 / 1856$ & $\begin{array}{l}27-02-07-01 \mathrm{~F} . \\
76\end{array}$ \\
\hline
\end{tabular}

91. This appendix was prepared by Spencer Toubia, KU Class of 2015, and is a rearrangement and reprinting of the citations contained in the Finding List to Territorial Kansas Online, a list prepared by the Kansas State Historical Society. 


\begin{tabular}{|c|c|c|c|c|}
\hline Assault & $1 \mathrm{st}$ & Wilson, John & $2 / 7 / 1855$ & $\begin{array}{l}27-02-08-01 \mathrm{~F} . \\
67\end{array}$ \\
\hline $\begin{array}{l}\text { Assault and } \\
\text { Battery }\end{array}$ & $3 \mathrm{rd}$ & Sweden, John & $8 / 24 / 1859$ & $\begin{array}{l}27-02-07-06 \mathrm{~F} . \\
32\end{array}$ \\
\hline $\begin{array}{l}\text { Assault and } \\
\text { Burglary }\end{array}$ & 2 nd & $\begin{array}{l}\text { Partridge, } \\
\text { Clayton }\end{array}$ & $8 / 10 / 1856$ & $\begin{array}{l}27-02-07-03 \mathrm{~F} . \\
33\end{array}$ \\
\hline $\begin{array}{l}\text { Assault and } \\
\text { Robbery }\end{array}$ & $1 \mathrm{st}, 2 \mathrm{nd}$ & $\begin{array}{l}\text { Cutler, Calvin \& } \\
\text { Dohman, Samuel } \\
\text { \& Lane, James }\end{array}$ & $9 / 20 / 1856$ & $\begin{array}{l}27-02-06-02 \mathrm{~F} . \\
46\end{array}$ \\
\hline $\begin{array}{l}\text { Assault on Henry } \\
\text { Gilmore }\end{array}$ & 2nd & Brown, John & $8 / 26 / 1856$ & $\begin{array}{l}27-02-05-07 \mathrm{~F} . \\
46\end{array}$ \\
\hline $\begin{array}{l}\text { Assault with } \\
\text { Intent to Kill }\end{array}$ & 2nd & Bailey, Calvin & $9 / 3 / 1857$ & $\begin{array}{l}27-02-05-07 \mathrm{~F} . \\
34\end{array}$ \\
\hline $\begin{array}{l}\text { Assault with } \\
\text { Intent to Kill }\end{array}$ & 2nd & Baldwin, David & $12 / 24 / 1854$ & $\begin{array}{l}27-02-05-06 \mathrm{~F} . \\
30\end{array}$ \\
\hline $\begin{array}{l}\text { Assault with } \\
\text { Intent to Kill }\end{array}$ & $1 \mathrm{st}$ & Berry, William & $4 / 1860$ & $\begin{array}{l}27-02-05-06 \mathrm{~F} . \\
62\end{array}$ \\
\hline $\begin{array}{l}\text { Assault with } \\
\text { Intent to Kill }\end{array}$ & 2nd & $\begin{array}{l}\text { Boyington, } \\
\text { Henry }\end{array}$ & $7 / 15 / 1857$ & $\begin{array}{l}27-02-05-07 \mathrm{~F} . \\
23\end{array}$ \\
\hline $\begin{array}{l}\text { Assault with } \\
\text { Intent to Kill }\end{array}$ & 2nd & Cumming, John & $5 / 10 / 1856$ & $\begin{array}{l}27-02-06-02 \mathrm{~F} . \\
39\end{array}$ \\
\hline $\begin{array}{l}\text { Assault with } \\
\text { Intent to Kill }\end{array}$ & 3rd & Curry, James & 9/15/1857 & $\begin{array}{l}27-02-06-02 \mathrm{~F} . \\
41\end{array}$ \\
\hline $\begin{array}{l}\text { Assault with } \\
\text { Intent to Kill }\end{array}$ & 2 nd & Davis, A.D.F. & $5 / 10 / 1856$ & $\begin{array}{l}27-02-06-03 \mathrm{~F} . \\
09\end{array}$ \\
\hline $\begin{array}{l}\text { Assault with } \\
\text { Intent to Kill }\end{array}$ & $1 \mathrm{st}$ & Day, Corlews & $9 / 12 / 1856$ & $\begin{array}{l}27-02-06-03 \mathrm{~F} . \\
17\end{array}$ \\
\hline $\begin{array}{l}\text { Assault with } \\
\text { Intent to Kill }\end{array}$ & 2nd & $\begin{array}{l}\text { Edwards, } \\
\text { Sanford }\end{array}$ & $9 / 23 / 1857$ & $\begin{array}{l}27-02-06-04 \mathrm{~F} . \\
03\end{array}$ \\
\hline $\begin{array}{l}\text { Assault with } \\
\text { Intent to Kill }\end{array}$ & $1 \mathrm{st}$ & Evans, David & $5 / 1 / 1856$ & $\begin{array}{l}27-02-06-04 \mathrm{~F} . \\
13\end{array}$ \\
\hline $\begin{array}{l}\text { Assault with } \\
\text { Intent to Kill }\end{array}$ & 3rd & Fizer, Archie & $6 / 10 / 1858$ & $\begin{array}{l}27-02-06-04 \mathrm{~F} . \\
36\end{array}$ \\
\hline $\begin{array}{l}\text { Assault with } \\
\text { Intent to Kill }\end{array}$ & 2nd & $\begin{array}{l}\text { Gagham, } \\
\text { William }\end{array}$ & $11 / 16 / 1855$ & $\begin{array}{l}27-02-06-05 \mathrm{~F} . \\
02\end{array}$ \\
\hline $\begin{array}{l}\text { Assault with } \\
\text { Intent to Kill }\end{array}$ & 2nd & $\begin{array}{l}\text { Gagham, } \\
\text { William }\end{array}$ & $12 / 1855$ & $\begin{array}{l}27-02-06-05 \mathrm{~F} . \\
01\end{array}$ \\
\hline $\begin{array}{l}\text { Assault with } \\
\text { Intent to Kill }\end{array}$ & $1 \mathrm{st}$ & $\begin{array}{l}\text { Hayes, Homer \& } \\
\text { W.J. Painter }\end{array}$ & $8 / 12 / 1856$ & $\begin{array}{l}27-02-06-05 \mathrm{~F} . \\
76\end{array}$ \\
\hline $\begin{array}{l}\text { Assault with } \\
\text { Intent to Kill }\end{array}$ & $1 \mathrm{st}$ & Kendal, Edward & $9 / 4 / 1856$ & $\begin{array}{l}27-02-06-07 \mathrm{~F} . \\
38\end{array}$ \\
\hline $\begin{array}{l}\text { Assault with } \\
\text { Intent to Kill }\end{array}$ & 2nd & Lockname, John & $1 / 5 / 1859$ & $\begin{array}{l}27-02-07-01 \mathrm{~F} . \\
29\end{array}$ \\
\hline $\begin{array}{l}\text { Assault with } \\
\text { Intent to Kill }\end{array}$ & 2nd & Lowry, John & $5 / 15 / 1858$ & $\begin{array}{l}27-02-07-01 \mathrm{~F} . \\
38\end{array}$ \\
\hline $\begin{array}{l}\text { Assault with } \\
\text { Intent to Kill }\end{array}$ & 2nd & Luther, A. & $3 / 26 / 1858$ & $\begin{array}{l}27-02-07-01 \mathrm{~F} . \\
43\end{array}$ \\
\hline $\begin{array}{l}\text { Assault with } \\
\text { Intent to Kill }\end{array}$ & $1 \mathrm{st}$ & $\begin{array}{l}\text { McKnown, } \\
\text { Robert }\end{array}$ & $9 / 4 / 1856$ & $\begin{array}{l}27-02-07-01 \mathrm{~F} . \\
89\end{array}$ \\
\hline $\begin{array}{l}\text { Assault with } \\
\text { Intent to Kill }\end{array}$ & Douglas Cnty. & $\begin{array}{l}\text { Merchison, } \\
\text { Keenion }\end{array}$ & ? & $\begin{array}{l}27-02-07-02 \mathrm{~F} . \\
02\end{array}$ \\
\hline $\begin{array}{l}\text { Assault with } \\
\text { Intent to Kill }\end{array}$ & $?$ & Owens, Haratio & $11 / 30 / 1854$ & $\begin{array}{l}27-02-07-03 \mathrm{~F} . \\
19\end{array}$ \\
\hline $\begin{array}{l}\text { Assault with } \\
\text { Intent to Kill }\end{array}$ & Jackson Cnty. & Puckett, Henry & $9 / 1858$ & $\begin{array}{l}27-02-07-03 \mathrm{~F} . \\
79\end{array}$ \\
\hline
\end{tabular}




\begin{tabular}{|c|c|c|c|c|}
\hline $\begin{array}{l}\text { Assault with } \\
\text { Intent to Kill }\end{array}$ & 2nd & Realf, Richard & $12 / 9 / 1856$ & $\begin{array}{l}\text { 27-02-07-04 F. } \\
05\end{array}$ \\
\hline $\begin{array}{l}\text { Assault with } \\
\text { Intent to Kill }\end{array}$ & 2nd & $\begin{array}{l}\text { Roarbacker, } \\
\text { Jacob }\end{array}$ & $4 / 1 / 1856$ & $\begin{array}{l}27-02-07-04 \mathrm{~F} . \\
42\end{array}$ \\
\hline $\begin{array}{l}\text { Assault with } \\
\text { Intent to Kill }\end{array}$ & $1 \mathrm{st}$ & Stowell, Martin & $8 / 15 / 1856$ & $\begin{array}{l}27-02-07-06 \mathrm{~F} . \\
24\end{array}$ \\
\hline $\begin{array}{l}\text { Assault with } \\
\text { Intent to Kill }\end{array}$ & $1 \mathrm{st}$ & Thompson, Jay & $5 / 1 / 1856$ & $\begin{array}{l}27-02-07-06 \mathrm{~F} . \\
53\end{array}$ \\
\hline $\begin{array}{l}\text { Assault with } \\
\text { Intent to Kill }\end{array}$ & 2nd & $\begin{array}{l}\text { Thompson, } \\
\text { William }\end{array}$ & $4 / 3 / 1858$ & $\begin{array}{l}27-02-07-06 \mathrm{~F} . \\
56\end{array}$ \\
\hline $\begin{array}{l}\text { Assault with } \\
\text { Intent to Kill }\end{array}$ & $1 \mathrm{st}$ & Walker, Samuel & $12 / 22 / 1856$ & $\begin{array}{l}27-02-07-07 \mathrm{~F} . \\
38\end{array}$ \\
\hline $\begin{array}{l}\text { Assault with } \\
\text { Intent to Kill }\end{array}$ & Douglas Cnty. & Wells, F. & $9 / 10 / 1856$ & $\begin{array}{l}27-02-08-01 \mathrm{~F} . \\
26\end{array}$ \\
\hline $\begin{array}{l}\text { Attack on } \\
\text { Franklin }\end{array}$ & $?$ & $\begin{array}{l}\text { Brown, John \& } \\
\text { Lenhart \& Abbot } \\
\text { \& Walker }\end{array}$ & $8 / 26 / 1856$ & $\begin{array}{l}27-02-05-07 \mathrm{~F} . \\
45\end{array}$ \\
\hline $\begin{array}{l}\text { Attack on Martin } \\
\text { White's House }\end{array}$ & 2nd & Holmes & $10 / 24 / 1856$ & $\begin{array}{l}27-02-06-06 \mathrm{~F} . \\
15\end{array}$ \\
\hline $\begin{array}{l}\text { Attempt to } \\
\text { Rescue a } \\
\text { Fugitive }\end{array}$ & $1 \mathrm{st}$ & $\begin{array}{l}\text { Anthondy, } \\
\text { Daniel }\end{array}$ & $6 / 1859$ & $\begin{array}{l}27-02-05-06 \mathrm{~F} . \\
17\end{array}$ \\
\hline $\begin{array}{l}\text { Attempt to } \\
\text { Rescue a } \\
\text { Fugitive }\end{array}$ & $1 \mathrm{st}$ & Gardiner, George & $6 / 28 / 1859$ & $\begin{array}{l}27-02-06-05 \mathrm{~F} . \\
06\end{array}$ \\
\hline $\begin{array}{l}\text { Attempt to } \\
\text { Rescue a } \\
\text { Fugitive }\end{array}$ & $1 \mathrm{st}$ & Weld, Lewis & $6 / 28 / 1859$ & $\begin{array}{l}27-02-08-01 \mathrm{~F} . \\
24\end{array}$ \\
\hline $\begin{array}{l}\text { Attempt to } \\
\text { Rescue a } \\
\text { Fugitive }\end{array}$ & $1 \mathrm{st}$ & Williams, James & $6 / 28.1859$ & $\begin{array}{l}27-02-08-01 \mathrm{~F} . \\
50\end{array}$ \\
\hline $\begin{array}{l}\text { Battery with } \\
\text { Intent to Kill }\end{array}$ & $1 \mathrm{st}$ & Emery, Thomas & $4 / 30 / 1855$ & $\begin{array}{l}27-02-06-04 \mathrm{~F} . \\
10\end{array}$ \\
\hline Breach of Bond & 2nd & Crane, Samuel & $9 / 12 / 1856$ & $\begin{array}{l}27-02-06-02 \mathrm{~F} . \\
30\end{array}$ \\
\hline Breach of Bond & 2nd & Jones, Samuel & $5 / 18 / 1859$ & $\begin{array}{l}27-02-06-07 \mathrm{~F} . \\
19\end{array}$ \\
\hline Breach of Peace & 2nd & Fascal, Fredrick & $10 / 27 / 1856$ & $\begin{array}{l}27-02-06-04 \mathrm{~F} . \\
25\end{array}$ \\
\hline Breach of Peace & 1st, 2nd & Wills, Wills & $3 / 5 / 1857$ & $\begin{array}{l}27-02-08-01 \mathrm{~F} . \\
61\end{array}$ \\
\hline Burglary & $1 \mathrm{st}$ & Crane, Hiram & $9 / 12 / 1856$ & $\begin{array}{l}27-02-06-02 \mathrm{~F} . \\
27\end{array}$ \\
\hline Burglary & $1 \mathrm{st}$ & $\begin{array}{l}\text { Giddings, } \\
\text { Richard }\end{array}$ & $10 / 17 / 1856$ & $\begin{array}{l}27-02-06-05 \mathrm{~F} . \\
16\end{array}$ \\
\hline Burglary & 2nd & $\begin{array}{l}\text { Partridge, } \\
\text { William }\end{array}$ & $10 / 22 / 1856$ & $\begin{array}{l}27-02-07-03 \mathrm{~F} . \\
34\end{array}$ \\
\hline $\begin{array}{l}\text { Burglary and } \\
\text { Larceny }\end{array}$ & 2 nd & Hilbourn, Henry & $10 / 24 / 1856$ & $\begin{array}{l}27-02-06-07 \mathrm{~F} . \\
47\end{array}$ \\
\hline $\begin{array}{l}\text { Capture of } \\
\text { Franklin \& } \\
\text { Hickory Point }\end{array}$ & 2nd & Kagle, John & $9 / 25 / 1856$ & $\begin{array}{l}27-02-06-07 \mathrm{~F} . \\
23\end{array}$ \\
\hline Card Betting & 2nd & Barber, William & $2 / 15 / 1856$ & $\begin{array}{l}27-02-05-06 \mathrm{~F} . \\
42\end{array}$ \\
\hline Cattle Larceny & 3rd & Conly, James & $5 / 1860$ & $\begin{array}{l}27-02-06-02 \mathrm{~F} . \\
14\end{array}$ \\
\hline
\end{tabular}


1184

KANSAS LAW REVIEW

[Vol. 62

\begin{tabular}{|c|c|c|c|c|}
\hline Conspiracy & $2 \mathrm{md}$ & Brown, O C & 1856 & $\begin{array}{l}27-02-05-07 \mathrm{~F} . \\
51\end{array}$ \\
\hline Contempt & 2nd & Adams, Calvin & $3 / 4 / 59$ & $\begin{array}{l}27-02-05-06 \mathrm{~F} . \\
03\end{array}$ \\
\hline Contempt & 2 nd & Adkins, James & $9 / 1857$ & $\begin{array}{l}27-02-05-06 \mathrm{~F} . \\
20\end{array}$ \\
\hline Contempt & 2nd & Babcock, Carmi & $2 / 29 / 1860$ & $\begin{array}{l}27-02-05-06 \mathrm{~F} . \\
21\end{array}$ \\
\hline Contempt & 2nd & Bailey, David & $2 / 24 / 1859$ & $\begin{array}{l}27-02-05-06 \mathrm{~F} . \\
23\end{array}$ \\
\hline Contempt & 2nd & Bailey, T.J. & $5 / 3 / 1857$ & $\begin{array}{l}27-02-05-06 \mathrm{~F} . \\
26\end{array}$ \\
\hline Contempt & 2nd & $\begin{array}{l}\text { Banks, } \\
\text { Rosenburg }\end{array}$ & $7 / 25 / 1859$ & $\begin{array}{l}27-02-05-06 \mathrm{~F} . \\
40\end{array}$ \\
\hline Contempt & 2nd & Banks, Russel & $2 / 24 / 1859$ & $\begin{array}{l}27-02-05-06 \mathrm{~F} . \\
41\end{array}$ \\
\hline Contempt & 2nd & Barnes, Lansing & $2 / 24 / 1856$ & $\begin{array}{l}27-02-05-06 \mathrm{~F} . \\
44\end{array}$ \\
\hline Contempt & Jackson Cnty. & Bash, Israel & $4 / 26 / 1860$ & $\begin{array}{l}27-02-05-06 \mathrm{~F} . \\
45\end{array}$ \\
\hline Contempt & 2nd & Bassett, Owen & $8 / 13 / 1857$ & $\begin{array}{l}27-02-05-06 \mathrm{~F} . \\
47\end{array}$ \\
\hline Contempt & 2nd & $\begin{array}{l}\text { Bassinguest, } \\
\text { John }\end{array}$ & $7 / 15 / 1859$ & $\begin{array}{l}27-02-05-06 \mathrm{~F} . \\
49\end{array}$ \\
\hline Contempt & 2 nd & Baum, Lewis & $7 / 15 / 1859$ & $\begin{array}{l}27-02-05-06 \mathrm{~F} . \\
50\end{array}$ \\
\hline Contempt & 2nd & Bently, John & $7 / 16 / 1859$ & $\begin{array}{l}27-02-05-06 \mathrm{~F} . \\
58\end{array}$ \\
\hline Contempt & 2nd & $\begin{array}{l}\text { Bergeron, } \\
\text { Francis }\end{array}$ & $3 / 6 / 1860$ & $\begin{array}{l}27-02-05-06 \mathrm{~F} . \\
59\end{array}$ \\
\hline Contempt & 2nd & Bergeron, John & $3 / 6 / 1860$ & $\begin{array}{l}27-02-05-06 \mathrm{~F} . \\
60\end{array}$ \\
\hline Contempt & 2nd & Bigham, John & $3 / 6 / 1860$ & $\begin{array}{l}27-02-05-07 \mathrm{~F} . \\
07\end{array}$ \\
\hline Contempt & 2nd & $\begin{array}{l}\text { Bingham, } \\
\text { Thomas }\end{array}$ & $3 / 6 / 1860$ & $\begin{array}{l}27-02-05-07 \mathrm{~F} . \\
07\end{array}$ \\
\hline Contempt & 2nd & Bourne, John & $2 / 24 / 1859$ & $\begin{array}{l}27-02-05-07 \mathrm{~F} . \\
18\end{array}$ \\
\hline Contempt & 2nd & Bouton, W.W. & $11 / 1856$ & $\begin{array}{l}27-02-05-07 \mathrm{~F} . \\
20\end{array}$ \\
\hline Contempt & 2nd & Bowles, Jerome & $9 / 14 / 1857$ & $\begin{array}{l}27-02-05-07 \mathrm{~F} . \\
22\end{array}$ \\
\hline Contempt & 2nd & $\begin{array}{l}\text { Bradford, } \\
\text { Clayton }\end{array}$ & $7 / 15 / 1859$ & $\begin{array}{l}27-02-05-07 \mathrm{~F} . \\
27\end{array}$ \\
\hline Contempt & 2nd & Brass, William & $10 / 4 / 1858$ & $\begin{array}{l}27-02-05-07 \mathrm{~F} . \\
30\end{array}$ \\
\hline Contempt & 2nd & Brewer, James & $11 / 1859$ & $\begin{array}{l}27-02-05-07 \mathrm{~F} . \\
33\end{array}$ \\
\hline Contempt & 2nd & Brooke, James & $2 / 24 / 1857$ & $\begin{array}{l}27-02-05-07 \mathrm{~F} . \\
35\end{array}$ \\
\hline Contempt & 2nd & $\begin{array}{l}\text { Brooke, } \\
\text { Theopolis }\end{array}$ & $2 / 24 / 1859$ & $\begin{array}{l}27-02-05-07 \mathrm{~F} . \\
36\end{array}$ \\
\hline Contempt & 2nd & Brown, Garwood & $2 / 24 / 1859$ & $\begin{array}{l}27-02-05-07 \mathrm{~F} . \\
38\end{array}$ \\
\hline Contempt & 2nd & Brown, George & $8 / 18 / 1857$ & $\begin{array}{l}27-02-05-07 \mathrm{~F} . \\
39\end{array}$ \\
\hline
\end{tabular}




\begin{tabular}{|c|c|c|c|c|}
\hline Contempt & 2 nd & Brown, John Jr. & $8 / 3 / 1856$ & $\begin{array}{l}27-02-05-07 \mathrm{~F} . \\
48\end{array}$ \\
\hline Contempt & $2 \mathrm{nd}$ & Brown, William & 1860 & $\begin{array}{l}27-02-05-07 \mathrm{~F} . \\
41\end{array}$ \\
\hline Contempt & $2 \mathrm{nd}$ & Bruner, Franklin & $2 / 1859$ & $\begin{array}{l}27-02-05-07 \mathrm{~F} . \\
52\end{array}$ \\
\hline Contempt & 2 nd & Buntschien, C & $3 / 1859$ & $\begin{array}{l}27-02-05-07 \mathrm{~F} . \\
58\end{array}$ \\
\hline Contempt & 1 st & Burke, Thomas & $8 / 22 / 1859$ & $\begin{array}{l}27-02-05-07 \mathrm{~F} . \\
62\end{array}$ \\
\hline Contempt & $2 \mathrm{nd}$ & Burwick, George & $9 / 19 / 1857$ & $\begin{array}{l}27-02-05-07 \mathrm{~F} . \\
63\end{array}$ \\
\hline Contempt & 2 nd & Butterfield, D.A. & $11 / 1859$ & $\begin{array}{l}27-02-05-07 \mathrm{~F} . \\
64\end{array}$ \\
\hline Contempt & 2 nd & Cameron, Hugh & $10 / 5 / 1857$ & $\begin{array}{l}27-02-06-01 \mathrm{~F} . \\
26\end{array}$ \\
\hline Contempt & 2 nd & Campbell, James & 9/14/1857 & $\begin{array}{l}27-02-06-01 \mathrm{~F} . \\
27\end{array}$ \\
\hline Contempt & $2 \mathrm{nd}$ & Campbell, James & 9/29/1857 & $\begin{array}{l}27-02-06-01 \mathrm{~F} . \\
28\end{array}$ \\
\hline Contempt & $2 \mathrm{nd}$ & $\begin{array}{l}\text { Carpenter, John } \\
\text { F. }\end{array}$ & $3 / 14 / 1860$ & $\begin{array}{l}27-02-06-01 \mathrm{~F} . \\
08\end{array}$ \\
\hline Contempt & 2 nd & Carrol, John & $2 / 14 / 1857$ & $\begin{array}{l}27-02-06-01 \mathrm{~F} . \\
11\end{array}$ \\
\hline Contempt & 2 nd & Childs, Seth & $5 / 9 / 1859$ & $\begin{array}{l}27-02-06-01 \mathrm{~F} . \\
18\end{array}$ \\
\hline Contempt & 2 nd & $\begin{array}{l}\text { Christian, } \\
\text { William }\end{array}$ & 9/14/1857 & $\begin{array}{l}27-02-06-01 \mathrm{~F} . \\
19\end{array}$ \\
\hline Contempt & $2 \mathrm{nd}$ & Clark, Henry & $3 / 6 / 1860$ & $\begin{array}{l}27-02-06-01 \mathrm{~F} . \\
34\end{array}$ \\
\hline Contempt & Douglas Cnty. & Clary, John & $5 / 26 / 1856$ & $\begin{array}{l}27-02-06-01 \mathrm{~F} . \\
40\end{array}$ \\
\hline Contempt & 2 nd & Cliboux, Louis & $3 / 6 / 1860$ & $\begin{array}{l}27-02-06-01 \mathrm{~F} . \\
44\end{array}$ \\
\hline Contempt & 2 nd & Cole, George & $8 / 28 / 1856$ & $\begin{array}{l}27-02-06-02 \mathrm{~F} . \\
06\end{array}$ \\
\hline Contempt & $2 \mathrm{~ns}$ & Cole, Joseph & $2 / 28 / 1859$ & $\begin{array}{l}27-02-06-02 \mathrm{~F} . \\
08\end{array}$ \\
\hline Contempt & $3 \mathrm{rd}$ & Corbey, Burnett & $10 / 9 / 1858$ & $\begin{array}{l}27-02-06-02 \mathrm{~F} . \\
21\end{array}$ \\
\hline Contempt & 2 nd & Corby, Q.T. & $5 / 4 / 1859$ & $\begin{array}{l}27-02-06-02 \mathrm{~F} . \\
19\end{array}$ \\
\hline Contempt & 2 nd & Corlew, Robert & $2 / 9 / 1858$ & $\begin{array}{l}27-02-06-02 \mathrm{~F} . \\
20\end{array}$ \\
\hline Contempt & 2 nd & $\begin{array}{l}\text { Corner, } \\
\text { Lafayette }\end{array}$ & $10 / 9 / 1858$ & $\begin{array}{l}27-02-06-02 \mathrm{~F} . \\
15\end{array}$ \\
\hline Contempt & $2 \mathrm{nd}$ & Costa, Lewis D & $3 / 6 / 1860$ & $\begin{array}{l}27-02-06-02 \mathrm{~F} . \\
22\end{array}$ \\
\hline Contempt & 2 nd & Danewood, John & $5 / 28 / 1860$ & $\begin{array}{l}27-02-06-03 \mathrm{~F} . \\
01\end{array}$ \\
\hline Contempt & 2nd & Davenport, R.M. & $3 / 3 / 1859$ & $\begin{array}{l}27-02-06-03 \mathrm{~F} . \\
08\end{array}$ \\
\hline Contempt & 2 nd & Davis, James & 9/29/57 & $\begin{array}{l}27-02-06-03 \mathrm{~F} . \\
14\end{array}$ \\
\hline Contempt & 2 nd & Davis, James & $3 / 9 / 1859$ & $\begin{array}{l}27-02-06-03 \mathrm{~F} . \\
13\end{array}$ \\
\hline
\end{tabular}


1186

KANSAS LAW REVIEW

[Vol. 62

\begin{tabular}{|c|c|c|c|c|}
\hline Contempt & 2nd & Davis, John & $9 / 24 / 1857$ & $\begin{array}{l}27-02-06-03 \mathrm{~F} . \\
15\end{array}$ \\
\hline Contempt & 2nd & Davis, S.H. & $9 / 14 / 1857$ & $\begin{array}{l}27-02-06-03 \mathrm{~F} . \\
16\end{array}$ \\
\hline Contempt & 2 nd & Douglas, G.C. & $7 / 15 / 1859$ & $\begin{array}{l}27-02-06-03 \mathrm{~F} . \\
37\end{array}$ \\
\hline Contempt & 2nd & Doyle, Daniel & $3 / 24 / 1859$ & $\begin{array}{l}27-02-06-03 \mathrm{~F} . \\
45\end{array}$ \\
\hline Contempt & 2nd & Dudley, Guiford & $6 / 14 / 1856$ & $\begin{array}{l}27-02-06-03 \mathrm{~F} . \\
49\end{array}$ \\
\hline Contempt & 2nd & Duncan, Charles & $7 / 16 / 1859$ & $\begin{array}{l}27-02-06-03 \mathrm{~F} . \\
53\end{array}$ \\
\hline Contempt & 2nd & Eagle, James & $11 / 5 / 1856$ & $\begin{array}{l}27-02-06-04 \mathrm{~F} . \\
01\end{array}$ \\
\hline Contempt & 2nd & Eaton, Charles & $10 / 9 / 1857$ & $\begin{array}{l}27-02-06-04 \mathrm{~F} . \\
02\end{array}$ \\
\hline Contempt & 2nd & Ellery, William & $6 / 21 / 1860$ & $\begin{array}{l}27-02-06-04 \mathrm{~F} . \\
05\end{array}$ \\
\hline Contempt & 2nd & Elliott, Isaiah & $3 / 6 / 1860$ & $\begin{array}{l}27-02-06-04 \mathrm{~F} . \\
07\end{array}$ \\
\hline Contempt & 2nd & Elliott, Jessie & $9 / 1858$ & $\begin{array}{l}27-02-06-04 \mathrm{~F} . \\
08\end{array}$ \\
\hline Contempt & Douglas Cnty. & Emery, J.S. & $7 / 26 / 1856$ & $\begin{array}{l}27-02-06-04 \mathrm{~F} . \\
11\end{array}$ \\
\hline Contempt & $1 \mathrm{st}$ & English, William & $7 / 23 / 1859$ & $\begin{array}{l}27-02-06-04 \mathrm{~F} . \\
12\end{array}$ \\
\hline Contempt & Jackson Cnty. & $\begin{array}{l}\text { Fairchild, } \\
\text { William }\end{array}$ & $10 / 1859$ & $\begin{array}{l}27-02-06-04 \mathrm{~F} . \\
19\end{array}$ \\
\hline Contempt & 2nd & Faris, J.J & $10 / 1 / 1860$ & $\begin{array}{l}27-02-06-04 \mathrm{~F} . \\
20\end{array}$ \\
\hline Contempt & 2nd & Farnham, M.G. & $3 / 5 / 1859$ & $\begin{array}{l}27-02-06-04 \mathrm{~F} . \\
21\end{array}$ \\
\hline Contempt & Jackson Cnty. & Ferrill, James & $4 / 26 / 1860$ & $\begin{array}{l}27-02-06-04 \mathrm{~F} . \\
28\end{array}$ \\
\hline Contempt & $3 \mathrm{rd}$ & Fields, Henry & $10 / 19 / 1858$ & $\begin{array}{l}27-02-06-04 \mathrm{~F} . \\
30\end{array}$ \\
\hline Contempt & 3rd & Fields, Richard & $10 / 19 / 1858$ & $\begin{array}{l}27-02-06-04 \mathrm{~F} . \\
31\end{array}$ \\
\hline Contempt & 2nd & Finger, Christian & $10 / 11 / 1858$ & $\begin{array}{l}27-02-06-04 \mathrm{~F} . \\
32\end{array}$ \\
\hline Contempt & 2nd & Fleshman, Perry & $9 / 28 / 1857$ & $\begin{array}{l}27-02-06-04 \mathrm{~F} . \\
39\end{array}$ \\
\hline Contempt & 2nd & Forbes, Elias & $3 / 28 / 1860$ & $\begin{array}{l}27-02-06-04 \mathrm{~F} . \\
42\end{array}$ \\
\hline Contempt & 2nd & Frapp, Gabriel & $4 / 6 / 1860$ & $\begin{array}{l}27-02-06-04 \mathrm{~F} . \\
48\end{array}$ \\
\hline Contempt & 2nd & Fuller, J.C. & $6 / 22 / 1860$ & $\begin{array}{l}27-02-06-04 \mathrm{~F} . \\
55\end{array}$ \\
\hline Contempt & 2nd & Fuller, Joseph & $4 / 6 / 1860$ & $\begin{array}{l}27-02-06-04 \mathrm{~F} . \\
59\end{array}$ \\
\hline Contempt & 2nd & Fuller, Perry & $4 / 26 / 1857$ & $\begin{array}{l}27-02-06-04 \mathrm{~F} . \\
60\end{array}$ \\
\hline Contempt & 2nd & Furrow, Devina & $3 / 14 / 1860$ & $\begin{array}{l}27-02-06-04 \mathrm{~F} . \\
68\end{array}$ \\
\hline Contempt & 2nd & Furrow, Devina & $7 / 15 / 1859$ & $\begin{array}{l}27-02-06-04 \mathrm{~F} . \\
69\end{array}$ \\
\hline
\end{tabular}




\begin{tabular}{|c|c|c|c|c|}
\hline Contempt & 2 nd & Gardner, O.B. & $2 / 22 / 1859$ & $\begin{array}{l}\text { 27-02-06-05 F. } \\
08\end{array}$ \\
\hline Contempt & $2 \mathrm{nd}$ & Gates, James & $6 / 12 / 1859$ & $\begin{array}{l}27-02-06-05 \mathrm{~F} . \\
11\end{array}$ \\
\hline Contempt & $2 \mathrm{nd}$ & Gidding, J.J. & $3 / 3 / 1859$ & $\begin{array}{l}27-02-06-05 \mathrm{~F} . \\
17\end{array}$ \\
\hline Contempt & 2 nd & Giddings, T.J. & $10 / 9 / 1857$ & $\begin{array}{l}27-02-06-05 \mathrm{~F} . \\
18\end{array}$ \\
\hline Contempt & Douglas Cnty. & $\begin{array}{l}\text { Gifford, } \\
\text { Hezekiah }\end{array}$ & $11 / 5 / 1859$ & $\begin{array}{l}27-02-06-05 \mathrm{~F} . \\
19\end{array}$ \\
\hline Contempt & $2 \mathrm{nd}$ & $\begin{array}{l}\text { Glenn, } \\
\text { Alexander }\end{array}$ & $2 / 24 / 1859$ & $\begin{array}{l}27-02-06-05 \mathrm{~F} . \\
26\end{array}$ \\
\hline Contempt & 2 nd & Golder, George & $2 / 24 / 1859$ & $\begin{array}{l}27-02-06-05 \mathrm{~F} . \\
27\end{array}$ \\
\hline Contempt & 2 nd & Goodell, John & $5 / 17 / 1859$ & $\begin{array}{l}27-02-06-05 \mathrm{~F} . \\
30\end{array}$ \\
\hline Contempt & 2 nd & Gover, Joel & $1 / 9 / 1858$ & $\begin{array}{l}27-02-06-05 \mathrm{~F} . \\
41\end{array}$ \\
\hline Contempt & 1 st & Graham & $7 / 26 / 1856$ & $\begin{array}{l}27-02-06-05 \mathrm{~F} . \\
32\end{array}$ \\
\hline Contempt & $2 \mathrm{nd}$ & Graham, Allen & $3 / 6 / 1860$ & $\begin{array}{l}27-02-06-05 \mathrm{~F} . \\
33\end{array}$ \\
\hline Contempt & $3 \mathrm{rd}$ & $\begin{array}{l}\text { Hamilton, } \\
\text { William }\end{array}$ & $6 / 23 / 1859$ & $\begin{array}{l}27-02-06-05 \mathrm{~F} . \\
47\end{array}$ \\
\hline Contempt & $?$ & Hansel, Salan & $2 / 24 / 1859$ & $\begin{array}{l}27-02-06-05 \mathrm{~F} . \\
51\end{array}$ \\
\hline Contempt & $?$ & Harper, William & $11 / 5 / 1856$ & $\begin{array}{l}27-02-06-05 \mathrm{~F} . \\
53\end{array}$ \\
\hline Contempt & $2 \mathrm{nd}$ & Hart, William & $3 / 14 / 1860$ & $\begin{array}{l}27-02-06-05 \mathrm{~F} . \\
59\end{array}$ \\
\hline Contempt & 2 nd & Henderson, C.C. & $2 / 24 / 1859$ & $\begin{array}{l}27-02-06-06 \mathrm{~F} . \\
02\end{array}$ \\
\hline Contempt & 2 nd & Henderson, C.C. & $3 / 5 / 1859$ & $\begin{array}{l}27-02-06-06 \mathrm{~F} . \\
02\end{array}$ \\
\hline Contempt & 2 nd & Herrall, William & $3 / 14 / 1860$ & $\begin{array}{l}27-02-06-06 \mathrm{~F} . \\
04\end{array}$ \\
\hline Contempt & $2 \mathrm{nd}$ & Hickox, J. & $3 / 4 / 1859$ & $\begin{array}{l}27-02-06-06 \mathrm{~F} . \\
07\end{array}$ \\
\hline Contempt & $2 \mathrm{nd}$ & Hicks, James & $2 / 24 / 1859$ & $\begin{array}{l}27-02-06-06 \mathrm{~F} . \\
06\end{array}$ \\
\hline Contempt & 2 nd & Hock, Samuel & $6 / 20 / 1860$ & $\begin{array}{l}27-02-06-06 \mathrm{~F} . \\
13\end{array}$ \\
\hline Contempt & 2 nd & Holmes, George & $10 / 4 / 1860$ & $\begin{array}{l}27-02-06-06 \mathrm{~F} . \\
16\end{array}$ \\
\hline Contempt & 2 nd & Holmes, William & $2 / 24 / 1859$ & $\begin{array}{l}27-02-06-06 \mathrm{~F} . \\
17\end{array}$ \\
\hline Contempt & $2 \mathrm{nd}$ & Hoover, Hiram & $3 / 14 / 1860$ & $\begin{array}{l}27-02-06-06 \mathrm{~F} . \\
18\end{array}$ \\
\hline Contempt & 2 nd & Hopkins, Eli & $8 / 12 / 1857$ & $\begin{array}{l}27-02-06-06 \mathrm{~F} . \\
19\end{array}$ \\
\hline Contempt & 2nd & Hoplins, L. & $2 / 23 / 1859$ & $\begin{array}{l}27-02-06-06 \mathrm{~F} . \\
21\end{array}$ \\
\hline Contempt & 2 nd & Houston. Samuel & $6 / 16 / 1859$ & $\begin{array}{l}27-02-06-06 \mathrm{~F} . \\
27\end{array}$ \\
\hline Contempt & 2 nd & Hunt, Asiel & $2 / 24 / 1859$ & $\begin{array}{l}27-02-06-06 \mathrm{~F} . \\
33\end{array}$ \\
\hline
\end{tabular}




\begin{tabular}{|c|c|c|c|c|}
\hline Contempt & 2nd & Hunt, Silas & $2 / 24 / 1859$ & $\begin{array}{l}27-02-06-06 \mathrm{~F} . \\
34\end{array}$ \\
\hline Contempt & 2nd & $\begin{array}{l}\text { Hutchinson, } \\
\text { G.W }\end{array}$ & $3 / 3 / 1859$ & $\begin{array}{l}27-02-06-06 \mathrm{~F} . \\
42\end{array}$ \\
\hline Contempt & Jackson Cnty. & $\begin{array}{l}\text { Jenison, } \\
\text { Theophilus }\end{array}$ & $4 / 26 / 1860$ & $\begin{array}{l}27-02-06-06 \mathrm{~F} . \\
57\end{array}$ \\
\hline Contempt & 2nd & $\begin{array}{l}\text { Johnson, } \\
\text { Benjamin }\end{array}$ & $5 / 10 / 1859$ & $\begin{array}{l}27-02-06-07 \mathrm{~F} . \\
03\end{array}$ \\
\hline Contempt & 2nd & Jones, Joseph & $4 / 21 / 1856$ & $\begin{array}{l}27-02-06-07 \mathrm{~F} . \\
17\end{array}$ \\
\hline Contempt & 3nd & Kelley, John & $3 / 14 / 1859$ & $\begin{array}{l}27-02-06-07 \mathrm{~F} . \\
31\end{array}$ \\
\hline Contempt & 2nd & Kelley, William & $6 / 26 / 1858$ & $\begin{array}{l}27-02-06-07 \mathrm{~F} . \\
35\end{array}$ \\
\hline Contempt & 2nd & Killum, Lewis & $3 / 6 / 1860$ & $\begin{array}{l}27-02-06-07 \mathrm{~F} . \\
52\end{array}$ \\
\hline Contempt & 2nd & $\begin{array}{l}\text { Kruykendall, } \\
\text { William }\end{array}$ & $2 / 24 / 1859$ & $\begin{array}{l}27-02-06-07 \mathrm{~F} . \\
62\end{array}$ \\
\hline Contempt & 2nd & Kunkle, Charles & $7 / 16 / 1859$ & $\begin{array}{l}27-02-06-07 \mathrm{~F} . \\
61\end{array}$ \\
\hline Contempt & $?$ & Lamb, William & $6 / 1 / 1860$ & $\begin{array}{l}27-02-07-01 \mathrm{~F} . \\
03\end{array}$ \\
\hline Contempt & 2nd & Laws, Alfred & $9 / 14 / 1857$ & $\begin{array}{l}27-02-07-01 \mathrm{~F} . \\
13\end{array}$ \\
\hline Contempt & 2nd & Laws, Alfred & $9 / 29 / 1857$ & $\begin{array}{l}27-02-07-01 \mathrm{~F} . \\
13\end{array}$ \\
\hline Contempt & 2nd & Lehay, John & $10 / 31 / 1857$ & $\begin{array}{l}\text { 27-02-07-01 F. } \\
11\end{array}$ \\
\hline Contempt & 2nd & Leroy, Joseph & $3 / 6 / 1860$ & $\begin{array}{l}27-02-07-01 \mathrm{~F} . \\
18\end{array}$ \\
\hline Contempt & 3rd & $\begin{array}{l}\text { Levassey, } \\
\text { William }\end{array}$ & $1 / 1 / 1859$ & $\begin{array}{l}27-02-07-01 \mathrm{~F} . \\
20\end{array}$ \\
\hline Contempt & $1 \mathrm{st}$ & Lewis, James & $7 / 23 / 1859$ & $\begin{array}{l}27-02-07-01 \mathrm{~F} . \\
21\end{array}$ \\
\hline Contempt & 3rd & $\begin{array}{l}\text { Lewis, William } \\
\text { \& Beck, M.D. \& } \\
\text { McDowell, John }\end{array}$ & $6 / 23 / 1859$ & $\begin{array}{l}27-02-07-01 \mathrm{~F} . \\
23\end{array}$ \\
\hline Contempt & 2nd & Logan, George & $3 / 14 / 1860$ & $\begin{array}{l}27-02-07-01 \mathrm{~F} . \\
30\end{array}$ \\
\hline Contempt & 2nd & Lovelace, Allen & $9 / 29 / 1857$ & $\begin{array}{l}27-02-07-01 \mathrm{~F} . \\
37\end{array}$ \\
\hline Contempt & 2nd & Luby, Michale & $2 / 24 / 1859$ & $\begin{array}{l}27-02-07-01 \mathrm{~F} . \\
39\end{array}$ \\
\hline Contempt & 1 st & Lucas, Edward & $4 / 3 / 1860$ & $\begin{array}{l}27-02-07-01 \mathrm{~F} . \\
40\end{array}$ \\
\hline Contempt & $1 \mathrm{st}$ & $\begin{array}{l}\text { Lucas, Edward } \\
\text { \& Topping, } \\
\text { James }\end{array}$ & $6 / 22 / 1859$ & $\begin{array}{l}27-02-07-01 \mathrm{~F} . \\
41\end{array}$ \\
\hline Contempt & $?$ & Lutz, William & $4 / 26 / 1860$ & $\begin{array}{l}27-02-07-01 \mathrm{~F} . \\
44\end{array}$ \\
\hline Contempt & 2nd & Marks, Andrew & $2 / 24 / 1859$ & $\begin{array}{l}27-02-07-01 \mathrm{~F} . \\
49\end{array}$ \\
\hline Contempt & 2nd & Marples, Abram & $2 / 24 / 1859$ & $\begin{array}{l}27-02-07-01 \mathrm{~F} . \\
47\end{array}$ \\
\hline Contempt & 2nd & Martin, Henry & $2 / 23 / 1859$ & $\begin{array}{l}\text { 27-02-07-01 F. } \\
51\end{array}$ \\
\hline
\end{tabular}




\begin{tabular}{|c|c|c|c|c|}
\hline Contempt & 2nd & Martin, William & $5 / 2 / 1857$ & $\begin{array}{l}27-02-07-01 \mathrm{~F} . \\
55\end{array}$ \\
\hline Contempt & 3 rd & Mathis, William & $10 / 24 / 1857$ & $\begin{array}{l}27-02-07-01 \mathrm{~F} . \\
56\end{array}$ \\
\hline Contempt & $?$ & McClure, James & $11 / 4 / 1859$ & $\begin{array}{l}27-02-07-01 \mathrm{~F} . \\
59\end{array}$ \\
\hline Contempt & 2nd & $\begin{array}{l}\text { McCulloch, } \\
\text { William }\end{array}$ & $7 / 15 / 1859$ & $\begin{array}{l}27-02-07-01 \mathrm{~F} . \\
63\end{array}$ \\
\hline Contempt & $1 \mathrm{st}$ & $\begin{array}{l}\text { McDevitt, } \\
\text { Thomas }\end{array}$ & $4 / 29 / 1858$ & $\begin{array}{l}27-02-07-01 \mathrm{~F} . \\
68\end{array}$ \\
\hline Contempt & 2nd & McDonald, Hugh & $3 / 6 / 1860$ & $\begin{array}{l}27-02-07-01 \mathrm{~F} . \\
71\end{array}$ \\
\hline Contempt & 2nd & $\begin{array}{l}\text { McFadden, } \\
\text { Andrew }\end{array}$ & $2 / 23 / 1859$ & $\begin{array}{l}27-02-07-01 \mathrm{~F} . \\
74\end{array}$ \\
\hline Contempt & 2 nd & $\begin{array}{l}\text { McKinney, } \\
\text { Charles }\end{array}$ & $11 / 22 / 1856$ & $\begin{array}{l}27-02-07-01 \mathrm{~F} . \\
79\end{array}$ \\
\hline Contempt & 2nd & McMullen, John & $7 / 26 / 1859$ & $\begin{array}{l}27-02-07-01 \mathrm{~F} . \\
88\end{array}$ \\
\hline Contempt & 2nd & Miller, Henry & $6 / 3 / 1856$ & $\begin{array}{l}27-02-07-02 \mathrm{~F} . \\
08\end{array}$ \\
\hline Contempt & 3 rd & Miller, John & $12 / 8 / 1859$ & $\begin{array}{l}27-02-07-02 \mathrm{~F} . \\
10\end{array}$ \\
\hline Contempt & Shawnee Cnty. & Miller, Joseph & $?$ & $\begin{array}{l}27-02-07-02 \mathrm{~F} . \\
11\end{array}$ \\
\hline Contempt & 2 nd & Miller, William & 9/14/1857 & $\begin{array}{l}27-02-07-02 \mathrm{~F} . \\
14\end{array}$ \\
\hline Contempt & 3rd & Miller, William & $12 / 1 / 1859$ & $\begin{array}{l}27-02-07-02 \mathrm{~F} . \\
13\end{array}$ \\
\hline Contempt & 2nd & Milligan, Samuel & $7 / 5 / 1861$ & $\begin{array}{l}27-02-07-02 \mathrm{~F} . \\
15\end{array}$ \\
\hline Contempt & $1 \mathrm{st}$ & Mills, Lafayette & $7 / 24 / 1858$ & $\begin{array}{l}27-02-07-02 \mathrm{~F} . \\
16\end{array}$ \\
\hline Contempt & 2nd & $\begin{array}{l}\text { Montague, } \\
\text { George }\end{array}$ & $6 / 20 / 1860$ & $\begin{array}{l}27-02-07-02 \mathrm{~F} . \\
33\end{array}$ \\
\hline Contempt & 2nd & Moore, Hugh & $2 / 24 / 1859$ & $\begin{array}{l}27-02-07-02 \mathrm{~F} . \\
39\end{array}$ \\
\hline Contempt & 2nd & Moreatta, Dennis & $2 / 24 / 1859$ & $\begin{array}{l}27-02-07-02 \mathrm{~F} . \\
41\end{array}$ \\
\hline Contempt & 2nd & Moreland, David & $3 / 1 / 1860$ & $\begin{array}{l}27-02-07-02 \mathrm{~F} . \\
42\end{array}$ \\
\hline Contempt & 2nd & Morey, John & $7 / 16 / 1859$ & $\begin{array}{l}27-02-07-02 \mathrm{~F} . \\
43\end{array}$ \\
\hline Contempt & 2nd & Morrow, Robert & $2 / 29 / 1860$ & $\begin{array}{l}27-02-07-02 \mathrm{~F} . \\
49\end{array}$ \\
\hline Contempt & 2nd & Morrow, Robert & $3 / 3 / 1859$ & $\begin{array}{l}27-02-07-02 \mathrm{~F} . \\
48\end{array}$ \\
\hline Contempt & 2nd & Murphy, Michael & $2 / 24 / 1859$ & $\begin{array}{l}27-02-07-02 \mathrm{~F} . \\
57\end{array}$ \\
\hline Contempt & 2nd & Murry, John & $7 / 15 / 1859$ & $\begin{array}{l}27-02-07-02 \mathrm{~F} . \\
59\end{array}$ \\
\hline Contempt & 2nd & Myers, Charles & $3 / 6 / 1860$ & $\begin{array}{l}27-02-07-02 \mathrm{~F} . \\
62\end{array}$ \\
\hline Contempt & 2nd & Newell, Edward & $7 / 15 / 1859$ & $\begin{array}{l}27-02-07-03 \mathrm{~F} . \\
04\end{array}$ \\
\hline Contempt & 2nd & Northrup, John & $9 / 14 / 1857$ & $\begin{array}{l}27-02-07-03 \mathrm{~F} . \\
09\end{array}$ \\
\hline
\end{tabular}




\begin{tabular}{|c|c|c|c|c|}
\hline Contempt & 2nd & Northrup, John & $9 / 29 / 1857$ & $\begin{array}{l}27-02-07-03 \mathrm{~F} . \\
10\end{array}$ \\
\hline Contempt & 2nd & Norton, William & $11 / 5 / 1856$ & $\begin{array}{l}27-02-07-03 \mathrm{~F} . \\
11\end{array}$ \\
\hline Contempt & 2 nd & Norville, John & $3 / 6 / 1860$ & $\begin{array}{l}27-02-07-03 \mathrm{~F} . \\
12\end{array}$ \\
\hline Contempt & 2nd & Noteman, Barton & $2 / 23 / 1859$ & $\begin{array}{l}27-02-07-03 \mathrm{~F} . \\
13\end{array}$ \\
\hline Contempt & 2nd & $\begin{array}{l}\text { O'Donnel, } \\
\text { William }\end{array}$ & $2 / 29 / 1860$ & $\begin{array}{l}27-02-07-03 \mathrm{~F} . \\
14\end{array}$ \\
\hline Contempt & 2nd & Osgood, Charles & $2 / 23 / 1859$ & $\begin{array}{l}27-02-07-03 \mathrm{~F} . \\
16\end{array}$ \\
\hline Contempt & 2nd & Otey, Charles & $3 / 5 / 1859$ & $\begin{array}{l}27-02-07-03 \mathrm{~F} . \\
17\end{array}$ \\
\hline Contempt & Jackson Cnty. & Owen, Ambrose & $4 / 26 / 1860$ & $\begin{array}{l}27-02-07-03 \mathrm{~F} . \\
18\end{array}$ \\
\hline Contempt & 2nd & Packard, Cyrus & $2 / 24 / 1859$ & $\begin{array}{l}27-02-07-03 \mathrm{~F} . \\
21\end{array}$ \\
\hline Contempt & 2nd & Parham, Robert & $3 / 6 / 1860$ & $\begin{array}{l}27-02-07-03 \mathrm{~F} . \\
25\end{array}$ \\
\hline Contempt & 2nd & Parham, Robert & $7 / 13 / 1860$ & $\begin{array}{l}27-02-07-03 \mathrm{~F} . \\
26\end{array}$ \\
\hline Contempt & $1 \mathrm{st}$ & Parish, Isaac & $3 / 4 / 1859$ & $\begin{array}{l}27-02-07-03 \mathrm{~F} . \\
27\end{array}$ \\
\hline Contempt & 2nd & Peoria, Baptiste & $3 / 9 / 1859$ & $\begin{array}{l}27-02-07-03 \mathrm{~F} . \\
44\end{array}$ \\
\hline Contempt & 2nd & Phelps, Joseph & $3 / 6 / 1860$ & $\begin{array}{l}27-02-07-03 \mathrm{~F} . \\
52\end{array}$ \\
\hline Contempt & 2nd & Philips, James & $2 / 22 / 1859$ & $\begin{array}{l}27-02-07-03 \mathrm{~F} . \\
54\end{array}$ \\
\hline Contempt & 2nd & Philips, Joel & $2 / 24 / 1859$ & $\begin{array}{l}27-02-07-03 \mathrm{~F} . \\
55\end{array}$ \\
\hline Contempt & 2nd & $\begin{array}{l}\text { Pickering, } \\
\text { Richard }\end{array}$ & $2 / 24 / 1859$ & $\begin{array}{l}27-02-07-03 \mathrm{~F} . \\
59\end{array}$ \\
\hline Contempt & 2nd & Pipher, John & $5 / 9 / 1859$ & $\begin{array}{l}27-02-07-03 \mathrm{~F} . \\
64\end{array}$ \\
\hline Contempt & 2nd & Pitts, Strand & $11 / 1 / 1859$ & $\begin{array}{l}27-02-07-03 \mathrm{~F} . \\
66\end{array}$ \\
\hline Contempt & 2nd & Pursall, Charles & $7 / 16 / 1859$ & $\begin{array}{l}27-02-07-04 \mathrm{~F} . \\
81\end{array}$ \\
\hline Contempt & 2nd & Quarles, T.J. & $3 / 3 / 1859$ & $\begin{array}{l}27-02-07-04 \mathrm{~F} . \\
01\end{array}$ \\
\hline Contempt & 2nd & Reid, John & $9 / 13 / 1857$ & $\begin{array}{l}27-02-07-04 \mathrm{~F} . \\
14\end{array}$ \\
\hline Contempt & 2nd & Reid, Samuel & $9 / 14 / 1857$ & $\begin{array}{l}27-02-07-04 \mathrm{~F} . \\
15\end{array}$ \\
\hline Contempt & $?$ & Reynard, Asa & $9 / 26 / 1860$ & $\begin{array}{l}27-02-07-04 \mathrm{~F} . \\
18\end{array}$ \\
\hline Contempt & 2nd & Rice, Alfred & $10 / 11 / 1858$ & $\begin{array}{l}27-02-07-04 \mathrm{~F} . \\
19\end{array}$ \\
\hline Contempt & 2nd & Rice, C.B. & $5 / 30 / 1860$ & $\begin{array}{l}27-02-07-04 \mathrm{~F} . \\
21\end{array}$ \\
\hline Contempt & 2nd & Robbins, Charles & $5 / 17 / 1859$ & $\begin{array}{l}27-02-07-04 \mathrm{~F} . \\
31\end{array}$ \\
\hline Contempt & 2nd & $\begin{array}{l}\text { Robbins, } \\
\text { Thomas }\end{array}$ & $3 / 28 / 1860$ & $\begin{array}{l}27-02-07-04 \mathrm{~F} . \\
32\end{array}$ \\
\hline
\end{tabular}




\begin{tabular}{|c|c|c|c|c|}
\hline Contempt & $?$ & Rose, Samuel & $4 / 26 / 1860$ & $\begin{array}{l}27-02-07-04 \mathrm{~F} . \\
43\end{array}$ \\
\hline Contempt & 2nd & Rosenquint, John & $7 / 15 / 1859$ & $\begin{array}{l}27-02-07-04 \mathrm{~F} . \\
43\end{array}$ \\
\hline Contempt & 2nd & Rucker, James & $9 / 14 / 1857$ & $\begin{array}{l}27-02-07-04 \mathrm{~F} . \\
47\end{array}$ \\
\hline Contempt & $3 \mathrm{rd}$ & Rucker, James & $9 / 29 / 1857$ & $\begin{array}{l}27-02-07-04 \mathrm{~F} . \\
48\end{array}$ \\
\hline Contempt & 2 nd & Saber, S.H. & $5 / 6 / 1859$ & $\begin{array}{l}27-02-07-05 \mathrm{~F} . \\
01\end{array}$ \\
\hline Contempt & 2nd & Sablin, Louis & $8 / 17 / 1857$ & $\begin{array}{l}27-02-07-05 \mathrm{~F} . \\
02\end{array}$ \\
\hline Contempt & 2nd & Sabree, E.S. & $2 / 24 / 1859$ & $\begin{array}{l}27-02-07-05 \mathrm{~F} . \\
28\end{array}$ \\
\hline Contempt & $3 \mathrm{rd}$ & Sage, James & $12 / 1 / 1859$ & $\begin{array}{l}27-02-07-05 \mathrm{~F} . \\
04\end{array}$ \\
\hline Contempt & 2nd & Sampson, Truner & $3 / 4 / 1859$ & $\begin{array}{l}27-02-07-05 \mathrm{~F} . \\
08\end{array}$ \\
\hline Contempt & 2nd & Sanford & $7 / 15 / 1859$ & $\begin{array}{l}27-02-07-05 \mathrm{~F} . \\
10\end{array}$ \\
\hline Contempt & 2nd & Sewell, Ely & $7 / 15 / 1859$ & $\begin{array}{l}27-02-07-05 \mathrm{~F} . \\
30\end{array}$ \\
\hline Contempt & 2nd & Shanklin & $3 / 14 / 1860$ & $\begin{array}{l}27-02-07-05 \mathrm{~F} . \\
37\end{array}$ \\
\hline Contempt & 2nd & $\begin{array}{l}\text { Shannon, } \\
\text { Anthony }\end{array}$ & $2 / 23 / 1859$ & $\begin{array}{l}27-02-07-05 \mathrm{~F} . \\
38\end{array}$ \\
\hline Contempt & 2nd & Shaw, Cyrus & $10 / 31 / 1857$ & $\begin{array}{l}27-02-07-05 \mathrm{~F} . \\
39\end{array}$ \\
\hline Contempt & 2nd & Shields, Hiram & $3 / 3 / 1859$ & $\begin{array}{l}27-02-07-05 \mathrm{~F} . \\
41\end{array}$ \\
\hline Contempt & 2nd & Simcock, G.M & $7 / 15 / 1859$ & $\begin{array}{l}27-02-07-05 \mathrm{~F} . \\
32\end{array}$ \\
\hline Contempt & 2nd & Smarr & $3 / 5 / 1859$ & $\begin{array}{l}27-02-07-05 \mathrm{~F} . \\
48\end{array}$ \\
\hline Contempt & 2nd & Sodon, William & $3 / 6 / 1860$ & $\begin{array}{l}27-02-07-05 \mathrm{~F} . \\
62\end{array}$ \\
\hline Contempt & 2nd & Spicely, William & $2 / 22 / 1859$ & $\begin{array}{l}27-02-07-05 \mathrm{~F} . \\
67\end{array}$ \\
\hline Contempt & 2nd & Spicely, William & 9/14/1857 & $\begin{array}{l}27-02-07-05 \mathrm{~F} . \\
66\end{array}$ \\
\hline Contempt & 2nd & Spicely, William & $9 / 19 / 1857$ & $\begin{array}{l}27-02-07-05 \mathrm{~F} . \\
66\end{array}$ \\
\hline Contempt & 2nd & Spicer, Edward & $7 / 16 / 1859$ & $\begin{array}{l}27-02-07-05 \mathrm{~F} . \\
68\end{array}$ \\
\hline Contempt & 2nd & Sponable, John & $2 / 23 / 1859$ & $\begin{array}{l}27-02-07-05 \mathrm{~F} . \\
70\end{array}$ \\
\hline Contempt & 2nd & Staggs, Squire & $8 / 12 / 1857$ & $\begin{array}{l}27-02-07-06 \mathrm{~F} . \\
02\end{array}$ \\
\hline Contempt & 2nd & $\begin{array}{l}\text { Stephens, } \\
\text { Charles }\end{array}$ & $11 / 5 / 1856$ & $\begin{array}{l}27-02-07-06 \mathrm{~F} . \\
08\end{array}$ \\
\hline Contempt & 2nd & Stevens, Taylor & $3 / 4 / 1859$ & $\begin{array}{l}27-02-07-06 \mathrm{~F} . \\
11\end{array}$ \\
\hline Contempt & 2nd & Swartz, Isaac & $3 / 29 / 1860$ & $\begin{array}{l}27-02-07-06 \mathrm{~F} . \\
31\end{array}$ \\
\hline Contempt & $?$ & Tennant, John & $10 / 29 / 59$ & $\begin{array}{l}27-02-07-06 \mathrm{~F} . \\
42\end{array}$ \\
\hline
\end{tabular}




\begin{tabular}{|c|c|c|c|c|}
\hline Contempt & 2nd & $\begin{array}{l}\text { Thompson, } \\
\text { James }\end{array}$ & $3 / 5 / 1859$ & $\begin{array}{l}27-02-07-06 \mathrm{~F} . \\
52\end{array}$ \\
\hline Contempt & 2nd & $\begin{array}{l}\text { Thompson, } \\
\text { James }\end{array}$ & $5 / 1 / 1856$ & $\begin{array}{l}27-02-07-06 \mathrm{~F} . \\
52\end{array}$ \\
\hline Contempt & 2nd & $\begin{array}{l}\text { Todhunter, } \\
\text { James }\end{array}$ & $2 / 24 / 1859$ & $\begin{array}{l}27-02-07-07 \mathrm{~F} . \\
01\end{array}$ \\
\hline Contempt & 2nd & Totten, William & 9/14/1857 & $\begin{array}{l}27-02-07-07 \mathrm{~F} . \\
03\end{array}$ \\
\hline Contempt & 2nd & Tucker, Theron & $11 / 5 / 1856$ & $\begin{array}{l}27-02-07-07 \mathrm{~F} . \\
09\end{array}$ \\
\hline Contempt & 2nd & Tully, Jacob & $9 / 14 / 1857$ & $\begin{array}{l}27-02-07-07 \mathrm{~F} . \\
10\end{array}$ \\
\hline Contempt & 2nd & Uphan, David & $7 / 15 / 1859$ & $\begin{array}{l}27-02-07-07 \mathrm{~F} . \\
25\end{array}$ \\
\hline Contempt & 2nd & Vieux, Charles & $5 / 30 / 1860$ & $\begin{array}{l}27-02-07-07 \mathrm{~F} . \\
27\end{array}$ \\
\hline Contempt & 2nd & Voak & $33 / 3 / 1859$ & $\begin{array}{l}27-02-07-07 \mathrm{~F} . \\
28\end{array}$ \\
\hline Contempt & 2nd & Wallace, C.M. & $3 / 3 / 1859$ & $\begin{array}{l}27-02-08-01 \mathrm{~F} . \\
01\end{array}$ \\
\hline Contempt & 2nd & Ward, Allen & $6 / 28 / 1859$ & $\begin{array}{l}27-02-08-01 \mathrm{~F} . \\
06\end{array}$ \\
\hline Contempt & 2nd & Warren, James & $8 / 12 / 1857$ & $\begin{array}{l}27-02-08-01 \mathrm{~F} . \\
10\end{array}$ \\
\hline Contempt & 2nd & Wells, James & $10 / 9 / 1858$ & $\begin{array}{l}27-02-08-01 \mathrm{~F} . \\
29\end{array}$ \\
\hline Contempt & 2nd & Welsh, Moses & $2 / 24 / 1859$ & $\begin{array}{l}27-02-08-01 \mathrm{~F} . \\
31\end{array}$ \\
\hline Contempt & 2nd & Welsh, William & $3 / 6 / 1860$ & $\begin{array}{l}27-02-08-01 \mathrm{~F} . \\
32\end{array}$ \\
\hline Contempt & 2nd & West & $10 / 31 / 1857$ & $\begin{array}{l}27-02-08-01 \mathrm{~F} . \\
33\end{array}$ \\
\hline Contempt & 2nd & Williams, John & $3 / 6 / 1860$ & $\begin{array}{l}27-02-08-01 \mathrm{~F} . \\
54\end{array}$ \\
\hline Contempt & 2nd & $\begin{array}{l}\text { Williams, } \\
\text { William }\end{array}$ & $5 / 17 / 1856$ & $\begin{array}{l}27-02-08-01 \mathrm{~F} . \\
57\end{array}$ \\
\hline Contempt & 2nd & Wilson, James & $6 / 20 / 1860$ & $\begin{array}{l}27-02-08-01 \mathrm{~F} . \\
64\end{array}$ \\
\hline Contempt & $3 \mathrm{rd}$ & $\begin{array}{l}\text { Witcher, Tandy } \\
\text { \& Baugh, } \\
\text { Bartlett \& } \\
\text { Baugh, John }\end{array}$ & $10 / 13 / 1857$ & $\begin{array}{l}27-02-08-01 \mathrm{~F} . \\
73\end{array}$ \\
\hline Contempt & 2nd & $\begin{array}{l}\text { Witherington, } \\
\text { Charles }\end{array}$ & $5 / 1860$ & $\begin{array}{l}27-02-08-01 \mathrm{~F} . \\
74\end{array}$ \\
\hline Contempt & $3 \mathrm{rd}$ & Wolf, Thomas & $10 / 19 / 1858$ & $\begin{array}{l}27-02-08-01 \mathrm{~F} . \\
75\end{array}$ \\
\hline Contempt & 2nd & Wood, G.B & $11 / 5 / 1856$ & $\begin{array}{l}27-02-08-01 \mathrm{~F} . \\
76\end{array}$ \\
\hline Contempt & 2nd & Woodman, Levi & $6 / 22 / 1860$ & $\begin{array}{l}27-02-08-01 \mathrm{~F} . \\
79\end{array}$ \\
\hline Contempt & 2nd & Woodruff, T.P. & $3 / 14 / 1860$ & $\begin{array}{l}27-02-08-01 \mathrm{~F} . \\
80\end{array}$ \\
\hline Contempt & 2nd & Worden & $7 / 16 / 1859$ & $\begin{array}{l}27-02-08-01 \mathrm{~F} . \\
82\end{array}$ \\
\hline Contempt & 2nd & Allen, Lucas & 1859 & $\begin{array}{l}27-02-05-06 \mathrm{~F} . \\
12\end{array}$ \\
\hline
\end{tabular}




\begin{tabular}{|c|c|c|c|c|}
\hline Contempt & 2nd & Dare, B.F. & $10 / 1858$ & $\begin{array}{l}27-02-06-03 \mathrm{~F} . \\
05\end{array}$ \\
\hline Contempt & 2 nd & Dye, David & $2 / 22 / 1859$ & $\begin{array}{l}27-02-06-03 \mathrm{~F} . \\
56\end{array}$ \\
\hline Contempt & 2 nd & $\begin{array}{l}\text { Glazelbrook, } \\
\text { I.R.C. }\end{array}$ & $7 / 27 / 1858$ & $\begin{array}{l}27-02-06-05 \mathrm{~F} . \\
24\end{array}$ \\
\hline Contempt & 2 nd & Jacobs, William & $3 / 3 / 1859$ & $\begin{array}{l}27-02-06-06 \mathrm{~F} . \\
50\end{array}$ \\
\hline Contempt & 2 nd & Lord, William & 7/15/1859 & $\begin{array}{l}27-02-07-01 \mathrm{~F} . \\
10\end{array}$ \\
\hline Contempt & 2nd & Parkinson, John & $2 / 23 / 1859$ & $\begin{array}{l}27-02-07-03 \mathrm{~F} . \\
28\end{array}$ \\
\hline Contempt & 2nd & $\begin{array}{l}\text { Simmons, } \\
\text { William } \\
\end{array}$ & $6 / 28 / 1859$ & $\begin{array}{l}27-02-07-05 \mathrm{~F} . \\
33\end{array}$ \\
\hline Contempt & 2 nd & Sypes, William & $2 / 24 / 1859$ & $\begin{array}{l}27-02-07-06 \mathrm{~F} . \\
35\end{array}$ \\
\hline Contempt & Jackson Cnty. & Watters & $4 / 26 / 1860$ & $\begin{array}{l}27-02-08-01 \mathrm{~F} . \\
14\end{array}$ \\
\hline Contempt & 2 nd & Waysman, J.K. & 9/14/1857 & $\begin{array}{l}27-02-08-01 \mathrm{~F} . \\
18\end{array}$ \\
\hline Cost Recovery & 2 nd & $\begin{array}{l}\text { Partridge, } \\
\text { William }\end{array}$ & $1 / 9 / 1857$ & $\begin{array}{l}27-02-07-03 \mathrm{~F} . \\
36\end{array}$ \\
\hline Counterfeiting & 2nd & Clark, Thomas & $5 / 1 / 1860$ & $\begin{array}{l}27-02-06-01 \mathrm{~F} . \\
38\end{array}$ \\
\hline Counterfeiting & 1 st & Creech, William & $3 / 5 / 1860$ & $\begin{array}{l}27-02-06-02 \mathrm{~F} . \\
31\end{array}$ \\
\hline Counterfeiting & $1 \mathrm{st}$ & Doty, E.L & $9 / 13 / 1859$ & $\begin{array}{l}27-02-06-03 \mathrm{~F} . \\
36\end{array}$ \\
\hline Counterfeiting & $1 \mathrm{st}$ & Gray, Issac & $12 / 9 / 1856$ & $\begin{array}{l}27-02-06-05 \mathrm{~F} . \\
35\end{array}$ \\
\hline Counterfeiting & 2nd & Long, Jacob & $5 / 1 / 1860$ & $\begin{array}{l}27-02-07-01 \mathrm{~F} . \\
32\end{array}$ \\
\hline Counterfeiting & $3 \mathrm{rd}$ & Merril, Charles & $3 / 1 / 1860$ & $\begin{array}{l}27-02-07-02 \mathrm{~F} . \\
03\end{array}$ \\
\hline Counterfeiting & $1 \mathrm{st}$ & $\begin{array}{l}\text { Peppard, } \\
\text { William }\end{array}$ & $4 / 28 / 1858$ & $\begin{array}{l}27-02-07-03 \mathrm{~F} . \\
45\end{array}$ \\
\hline Counterfeiting & 2nd & Watters, Daniel & $6 / 5 / 1860$ & $\begin{array}{l}27-02-08-01 \mathrm{~F} . \\
04\end{array}$ \\
\hline Counterfeiting & 2nd & Watters, Daniel & $6 / 7 / 1860$ & $\begin{array}{l}27-02-08-01 \mathrm{~F} . \\
05\end{array}$ \\
\hline Cutting Timber & $1 \mathrm{st}$ & Leroux, Francis & $1 / 1 / 1859$ & $\begin{array}{l}27-02-07-01 \mathrm{~F} . \\
17\end{array}$ \\
\hline $\begin{array}{l}\text { Cutting Timber } \\
\text { off Indian Lands }\end{array}$ & $3 \mathrm{rd}$ & Long, Myron & $5 / 1 / 1857$ & $\begin{array}{l}27-02-07-01 \mathrm{~F} . \\
35\end{array}$ \\
\hline $\begin{array}{l}\text { Cutting Timber } \\
\text { on (Christian) } \\
\text { Indian Land }\end{array}$ & $1 \mathrm{st}$ & Fraker, William & $2 / 6 / 1858$ & $\begin{array}{l}27-02-06-04 \mathrm{~F} . \\
46\end{array}$ \\
\hline $\begin{array}{l}\text { Cutting Timber } \\
\text { on Indian Land }\end{array}$ & $3 \mathrm{rd}$ & $\begin{array}{l}\text { Butterworth, } \\
\text { Adam }\end{array}$ & $12 / 1859$ & $\begin{array}{l}27-02-05-07 \mathrm{~F} . \\
65\end{array}$ \\
\hline Debt & 3 rd & Jolly, Jeremiah & $11 / 28 / 1854$ & $\begin{array}{l}27-02-06-07 \mathrm{~F} . \\
11\end{array}$ \\
\hline Debtor Payment & 2nd & Hill, A.J. & $6 / 28 / 1859$ & $\begin{array}{l}27-02-06-06 \mathrm{~F} . \\
09\end{array}$ \\
\hline Debts & 2nd & $\begin{array}{l}\text { Bradbury, } \\
\text { Leonard }\end{array}$ & $6 / 28 / 1858$ & $\begin{array}{l}27-02-05-07 \mathrm{~F} . \\
26\end{array}$ \\
\hline
\end{tabular}




\begin{tabular}{|c|c|c|c|c|}
\hline $\begin{array}{l}\text { Disturbing the } \\
\text { Peace }\end{array}$ & 2nd & $\begin{array}{l}\text { Waysman, James } \\
\text { \& Edwards, } \\
\text { Robert \& Byler, } \\
\text { Alfred }\end{array}$ & $2 / 6 / 1856$ & $\begin{array}{l}27-02-08-01 \mathrm{~F} . \\
16\end{array}$ \\
\hline $\begin{array}{l}\text { Escape from } \\
\text { Officers }\end{array}$ & 2nd & Wood, S.N. & $4 / 19 / 1856$ & $\begin{array}{l}27-02-08-01 \mathrm{~F} . \\
78\end{array}$ \\
\hline $\begin{array}{l}\text { Failure to Keep } \\
\text { Peace }\end{array}$ & 2nd & Adams, Edward & $7 / 14 / 1857$ & $\begin{array}{l}27-02-05-06 \mathrm{~F} . \\
06\end{array}$ \\
\hline $\begin{array}{l}\text { Failure to Keep } \\
\text { Peace }\end{array}$ & 2nd & Bailey, John & $5 / 28 / 1857$ & $\begin{array}{l}27-02-05-06 \mathrm{~F} . \\
25\end{array}$ \\
\hline $\begin{array}{l}\text { Failure to Keep } \\
\text { Peace }\end{array}$ & 2nd & Chapman, John & $6 / 12 / 1856$ & $\begin{array}{l}27-02-06-01 \mathrm{~F} . \\
05\end{array}$ \\
\hline $\begin{array}{l}\text { Failure to Keep } \\
\text { Peace }\end{array}$ & 2nd & $\begin{array}{l}\text { Coffman, } \\
\text { Samuel }\end{array}$ & $5 / 9 / 1856$ & $\begin{array}{l}27-02-06-02 \mathrm{~F} . \\
03\end{array}$ \\
\hline $\begin{array}{l}\text { Failure to Keep } \\
\text { Peace }\end{array}$ & 2nd & Emerson, Joseph & $1 / 28 / 1857$ & $\begin{array}{l}27-02-06-04 \mathrm{~F} . \\
09\end{array}$ \\
\hline $\begin{array}{l}\text { Failure to Keep } \\
\text { Peace }\end{array}$ & 2nd & Jenner, Thomas & $10 / 13 / 1856$ & $\begin{array}{l}27-02-06-06 \mathrm{~F} . \\
56\end{array}$ \\
\hline $\begin{array}{l}\text { Failure to Keep } \\
\text { Peace }\end{array}$ & 2nd & Rice, Alfred & $5 / 5 / 1857$ & $\begin{array}{l}27-02-07-04 \mathrm{~F} . \\
20\end{array}$ \\
\hline $\begin{array}{l}\text { Failure to Keep } \\
\text { Peace }\end{array}$ & 2nd & Sims, Isaac & $2 / 6 / 1857$ & $\begin{array}{l}27-02-07-05 \mathrm{~F} . \\
35\end{array}$ \\
\hline Felony & $?$ & Harris, Bill & $12 / 22 / 1856$ & $\begin{array}{l}27-02-06-05 \mathrm{~F} . \\
54\end{array}$ \\
\hline $\begin{array}{l}\text { Felony (Shooting } \\
\text { a horse) }\end{array}$ & 2nd & Moffet, Charles & $1 / 16 / 1856$ & $\begin{array}{l}27-02-07-02 \mathrm{~F} . \\
25\end{array}$ \\
\hline Fighting & Douglas Cnty. & Newill & $9 / 23 / 1856$ & $\begin{array}{l}27-02-07-03 \mathrm{~F} . \\
03\end{array}$ \\
\hline $\begin{array}{l}\text { Forfeited } \\
\text { Recognizance }\end{array}$ & 2nd & Bibbee, Lucius & $11 / 5 / 1856$ & $\begin{array}{l}27-02-06-07 \mathrm{~F} . \\
43\end{array}$ \\
\hline $\begin{array}{l}\text { Forfeited } \\
\text { Recognizance }\end{array}$ & 3 rd & Dare, B.F. & $11 / 30 / 1859$ & $\begin{array}{l}27-02-06-03 \mathrm{~F} . \\
06\end{array}$ \\
\hline $\begin{array}{l}\text { Forfeited } \\
\text { Recognizance }\end{array}$ & 3rd & Dunbar, Henry & $4 / 19 / 1860$ & $\begin{array}{l}27-02-06-03 \mathrm{~F} . \\
52\end{array}$ \\
\hline $\begin{array}{l}\text { Forfeited } \\
\text { Recognizance }\end{array}$ & $1 \mathrm{st}$ & Fahey, Tomas & $9 / 22 / 1859$ & $\begin{array}{l}27-02-06-04 \mathrm{~F} . \\
18\end{array}$ \\
\hline $\begin{array}{l}\text { Forfeited } \\
\text { Recognizance }\end{array}$ & 2nd & Garrett, Charles & $2 / 9 / 1858$ & $\begin{array}{l}27-02-06-05 \mathrm{~F} . \\
09\end{array}$ \\
\hline $\begin{array}{l}\text { Forfeited } \\
\text { Recognizance }\end{array}$ & 2nd & Hayes, Charles & $1 / 5 / 1858$ & $\begin{array}{l}27-02-06-05 \mathrm{~F} . \\
74\end{array}$ \\
\hline $\begin{array}{l}\text { Forfeited } \\
\text { Recognizance }\end{array}$ & 2nd & $\begin{array}{l}\text { Hazeltine, } \\
\text { Francis }\end{array}$ & $2 / 8 / 1858$ & $\begin{array}{l}27-02-06-05 \mathrm{~F} . \\
77\end{array}$ \\
\hline $\begin{array}{l}\text { Forfeited } \\
\text { Recognizance }\end{array}$ & 2nd & $\begin{array}{l}\text { Hazelton, } \\
\text { William }\end{array}$ & $8 / 14 / 1858$ & $\begin{array}{l}27-02-06-05 \mathrm{~F} . \\
78\end{array}$ \\
\hline $\begin{array}{l}\text { Forfeited } \\
\text { Recognizance }\end{array}$ & $1 \mathrm{st}$ & Horst, Herman & $8 / 23 / 1859$ & $\begin{array}{l}27-02-06-06 \mathrm{~F} . \\
24\end{array}$ \\
\hline $\begin{array}{l}\text { Forfeited } \\
\text { Recognizance }\end{array}$ & 2nd & Jones, John & $9 / 14 / 1857$ & $\begin{array}{l}27-02-06-07 \mathrm{~F} . \\
16\end{array}$ \\
\hline $\begin{array}{l}\text { Forfeited } \\
\text { Recognizance }\end{array}$ & 2nd & Kagle, John & $1 / 21 / 1858$ & $\begin{array}{l}27-02-06-07 \mathrm{~F} . \\
25\end{array}$ \\
\hline $\begin{array}{l}\text { Forfeited } \\
\text { Recognizance }\end{array}$ & 2nd & Kagle, John & $9 / 14 / 1857$ & $\begin{array}{l}27-02-06-07 \mathrm{~F} . \\
24\end{array}$ \\
\hline
\end{tabular}




\begin{tabular}{|c|c|c|c|c|}
\hline $\begin{array}{l}\text { Forfeited } \\
\text { Recognizance }\end{array}$ & 2nd & $\begin{array}{l}\text { Kagle, John \& } \\
\text { Muffit, Orlando } \\
\text { \& Updegraff, } \\
\text { Derrick \& } \\
\text { Bonhem, Samuel } \\
\text { \& Shepherd, } \\
\text { Paul }\end{array}$ & $1 / 14 / 1858$ & $\begin{array}{l}27-02-06-07 \mathrm{~F} . \\
27\end{array}$ \\
\hline $\begin{array}{l}\text { Forfeited } \\
\text { Recognizance }\end{array}$ & 2nd & $\begin{array}{l}\text { Kibee. Lucius \& } \\
\text { Baysinger, Peter } \\
\text { \& Hill, Elizar }\end{array}$ & $3 / 26 / 1857$ & $\begin{array}{l}27-02-06-07 \mathrm{~F} . \\
44\end{array}$ \\
\hline $\begin{array}{l}\text { Forfeited } \\
\text { Recognizance }\end{array}$ & 2nd & $\begin{array}{l}\text { Lane, James \& } \\
\text { Shannon, Wilson } \\
\text { \& McIntosh, } \\
\text { David }\end{array}$ & $3 / 19 / 1860$ & $\begin{array}{l}\text { 27-02-07-01 F. } \\
08\end{array}$ \\
\hline $\begin{array}{l}\text { Forfeited } \\
\text { Recognizance }\end{array}$ & 2nd & $\begin{array}{l}\text { Lockhart, } \\
\text { Samuel \& Jones, } \\
\text { James }\end{array}$ & $1 / 7 / 1858$ & $\begin{array}{l}27-02-07-01 \mathrm{~F} . \\
26\end{array}$ \\
\hline $\begin{array}{l}\text { Forfeited } \\
\text { Recognizance }\end{array}$ & 2nd & $\begin{array}{l}\text { McKinney, } \\
\text { Wilder \& } \\
\text { Mattney, R.B \& } \\
\text { Reed, E.B. }\end{array}$ & $9 / 15 / 1857$ & $\begin{array}{l}27-02-07-01 \mathrm{~F} . \\
83\end{array}$ \\
\hline $\begin{array}{l}\text { Forfeited } \\
\text { Recognizance }\end{array}$ & 2nd & $\begin{array}{l}\text { McKnown, } \\
\text { Robert \& } \\
\text { McCole, Joseph, } \\
\text { Milne, David \& } \\
\text { Fiddling, Charles } \\
\text { \& Murphy, } \\
\text { Richard }\end{array}$ & $1 / 13 / 1858$ & $\begin{array}{l}\text { 27-02-07-01 F. } \\
90\end{array}$ \\
\hline $\begin{array}{l}\text { Forfeited } \\
\text { Recognizance }\end{array}$ & 2nd & $\begin{array}{l}\text { Miller, Josiah \& } \\
\text { Legate, James \& } \\
\text { Phillips, William } \\
\text { \& Goodwin, Joel }\end{array}$ & $1 / 23 / 1858$ & $\begin{array}{l}27-02-06-07 \mathrm{~F} . \\
26\end{array}$ \\
\hline $\begin{array}{l}\text { Forfeited } \\
\text { Recognizance }\end{array}$ & 2nd & $\begin{array}{l}\text { Moffet, Charles } \\
\text { \& Moffet, } \\
\text { Orlando \& Tyler, } \\
\text { Jehial }\end{array}$ & $1 / 15 / 1858$ & $\begin{array}{l}27-02-07-02 \mathrm{~F} . \\
29\end{array}$ \\
\hline $\begin{array}{l}\text { Forfeited } \\
\text { Recognizance }\end{array}$ & 2nd & $\begin{array}{l}\text { Moffet, Charles } \\
\text { \& Moffet, } \\
\text { Orlando \& Tyler, } \\
\text { Jehial } \\
\end{array}$ & $1 / 22 / 1858$ & $\begin{array}{l}27-02-07-02 \mathrm{~F} . \\
30\end{array}$ \\
\hline $\begin{array}{l}\text { Forfeited } \\
\text { Recognizance }\end{array}$ & 2nd & $\begin{array}{l}\text { Moffet, Charles } \\
\text { \& Moffet } \\
\text { Orlando \& Tyler, } \\
\text { Jehial }\end{array}$ & $12 / 29 / 1857$ & $\begin{array}{l}27-02-07-02 \mathrm{~F} . \\
27\end{array}$ \\
\hline $\begin{array}{l}\text { Forfeited } \\
\text { Recognizance }\end{array}$ & 2nd & Storm, John & $12 / 23 / 1857$ & $\begin{array}{l}27-02-07-06 \mathrm{~F} . \\
21\end{array}$ \\
\hline $\begin{array}{l}\text { Forfeited } \\
\text { Recognizance }\end{array}$ & 2nd & Walker, Isaiah & $2 / 9 / 1858$ & $\begin{array}{l}27-02-07-07 \mathrm{~F} . \\
34\end{array}$ \\
\hline $\begin{array}{l}\text { Forfeited } \\
\text { Recognizance }\end{array}$ & 2nd & Walker, Mathew & $2 / 9 / 1858$ & $\begin{array}{l}27-02-07-07 \mathrm{~F} . \\
35\end{array}$ \\
\hline Gambling & 2nd & $\begin{array}{l}\text { Carmichael, } \\
\text { Robert }\end{array}$ & $11 / 1856$ & $\begin{array}{l}27-02-06-01 \mathrm{~F} . \\
07\end{array}$ \\
\hline Gambling & $1 \mathrm{st}$ & Cook, Daniel & $4 / 1 / 1858$ & $\begin{array}{l}27-02-06-02 \mathrm{~F} . \\
17\end{array}$ \\
\hline Gambling & 2nd & Cummings, John & $3 / 10 / 1856$ & $\begin{array}{l}27-02-06-02 \mathrm{~F} . \\
38\end{array}$ \\
\hline
\end{tabular}




\begin{tabular}{|c|c|c|c|c|}
\hline Gambling & 2nd & $\begin{array}{l}\text { Diggens, } \\
\text { Christopher }\end{array}$ & $4 / 1 / 1856$ & $\begin{array}{l}27-02-06-03 \mathrm{~F} . \\
27\end{array}$ \\
\hline Gambling & 2nd & Hamilton, John & $5 / 1856$ & $\begin{array}{l}27-02-06-05 \mathrm{~F} . \\
46\end{array}$ \\
\hline Gambling & $1 \mathrm{st}$ & Hand, James & $4 / 1856$ & $\begin{array}{l}27-02-06-05 \mathrm{~F} . \\
48\end{array}$ \\
\hline Gambling & 2 nd & Hill, B.F & $3 / 1 / 1856$ & $\begin{array}{l}27-02-06-06 \mathrm{~F} . \\
10\end{array}$ \\
\hline Gambling & $3 \mathrm{rd}$ & Hughs, J.C. & $8 / 10 / 1856$ & $\begin{array}{l}27-02-06-06 \mathrm{~F} . \\
30\end{array}$ \\
\hline Gambling & $1 \mathrm{st}$ & Lablin, Louis & $4 / 1 / 1856$ & $\begin{array}{l}27-02-07-01 \mathrm{~F} . \\
02\end{array}$ \\
\hline Gambling & $1 \mathrm{st}$ & $\begin{array}{l}\text { Leonard, } \\
\text { Thomas }\end{array}$ & $9 / 1 / 1857$ & $\begin{array}{l}27-02-07-01 \mathrm{~F} . \\
16\end{array}$ \\
\hline Gambling & $1 \mathrm{st}$ & $\begin{array}{l}\text { Lockhart, } \\
\text { Samuel }\end{array}$ & $4 / 1 / 1856$ & $\begin{array}{l}27-02-07-01 \mathrm{~F} . \\
25\end{array}$ \\
\hline Gambling & 2nd & Lockname, John & $1 / 15 / 1857$ & $\begin{array}{l}27-02-07-01 \mathrm{~F} . \\
28\end{array}$ \\
\hline Gambling & 2nd & Lockname, John & $2 / 1 / 1857$ & $\begin{array}{l}27-02-07-01 \mathrm{~F} . \\
27\end{array}$ \\
\hline Gambling & 2nd & $\begin{array}{l}\text { Margrave, } \\
\text { William }\end{array}$ & $6 / 3 / 1856$ & $\begin{array}{l}27-02-07-01 \mathrm{~F} . \\
48\end{array}$ \\
\hline Gambling & 2nd & Martin, William & $5 / 2 / 1857$ & $\begin{array}{l}27-02-07-01 \mathrm{~F} . \\
53\end{array}$ \\
\hline Gambling & 2 nd & $\begin{array}{l}\text { McDonald, } \\
\text { William }\end{array}$ & $1 / 31 / 1857$ & $\begin{array}{l}27-02-07-01 \mathrm{~F} . \\
72\end{array}$ \\
\hline Gambling & 2nd & $\begin{array}{l}\text { McDonald, } \\
\text { William }\end{array}$ & $3 / 4 / 1857$ & $\begin{array}{l}27-02-07-01 \mathrm{~F} . \\
73\end{array}$ \\
\hline Gambling & 2nd & $\begin{array}{l}\text { McKinney, } \\
\text { Luther }\end{array}$ & $9 / 10 / 1856$ & $\begin{array}{l}27-02-07-01 \mathrm{~F} . \\
80\end{array}$ \\
\hline Gambling & 2nd & $\begin{array}{l}\text { McKinney, } \\
\text { Wilder }\end{array}$ & $4 / 1 / 1856$ & $\begin{array}{l}27-02-07-01 \mathrm{~F} . \\
82\end{array}$ \\
\hline Gambling & 2 nd & Miller & $5 / 4 / 1857$ & $\begin{array}{l}27-02-07-02 \mathrm{~F} . \\
04\end{array}$ \\
\hline Gambling & 2nd & Miller, A.J. & $10 / 9 / 1856$ & $\begin{array}{l}27-02-07-02 \mathrm{~F} . \\
06\end{array}$ \\
\hline Gambling & 2nd & Mitchel, John & $10 / 9 / 1856$ & $\begin{array}{l}27-02-07-02 \mathrm{~F} . \\
23\end{array}$ \\
\hline Gambling & 2nd & Mormon, James & $11 / 24 / 1856$ & $\begin{array}{l}27-02-07-02 \mathrm{~F} . \\
45\end{array}$ \\
\hline Gambling & $1 \mathrm{st}$ & Muer, James & $9 / 1857$ & $\begin{array}{l}27-02-07-02 \mathrm{~F} . \\
50\end{array}$ \\
\hline Gambling & 2nd & Murry, Church & $1 / 31 / 1857$ & $\begin{array}{l}27-02-07-02 \mathrm{~F} . \\
58\end{array}$ \\
\hline Gambling & 2nd & Oyager, William & $9 / 10 / 1856$ & $\begin{array}{l}27-02-07-03 \mathrm{~F} . \\
20\end{array}$ \\
\hline Gambling & 2nd & Patrick, Allen & $3 / 1 / 1857$ & $\begin{array}{l}27-02-07-03 \mathrm{~F} . \\
38\end{array}$ \\
\hline Gambling & 2nd & Patrick, Allen & $3 / 15 / 1857$ & $\begin{array}{l}27-02-07-03 \mathrm{~F} . \\
38\end{array}$ \\
\hline Gambling & 2nd & Phillips, David & $11 / 26 / 1856$ & $\begin{array}{l}27-02-07-03 \mathrm{~F} . \\
56\end{array}$ \\
\hline Gambling & 2nd & Phillips, David & $10 / 9 / 1856$ & $\begin{array}{l}27-02-07-03 \mathrm{~F} . \\
53\end{array}$ \\
\hline Gambling & 2nd & Preston, William & $5 / 1 / 1857$ & $\begin{array}{l}27-02-07-03 \mathrm{~F} . \\
77\end{array}$ \\
\hline
\end{tabular}




\begin{tabular}{|c|c|c|c|c|}
\hline Gambling & 1 st & $\begin{array}{l}\text { Rice, J.T. \& } \\
\text { Jumpo, Edward }\end{array}$ & $4 / 30 / 1858$ & $\begin{array}{l}27-02-07-04 \mathrm{~F} . \\
22\end{array}$ \\
\hline Gambling & 2nd & Stewart, Owen & $5 / 1 / 1857$ & $\begin{array}{l}27-02-07-06 \mathrm{~F} . \\
13\end{array}$ \\
\hline Gambling & $1 \mathrm{st}$ & Stewart, William & $4 / 1 / 1856$ & $\begin{array}{l}27-02-07-06 \mathrm{~F} . \\
15\end{array}$ \\
\hline Gambling & 2nd & Strickler, Hiram & $9 / 10 / 1856$ & $\begin{array}{l}27-02-07-06 \mathrm{~F} . \\
26\end{array}$ \\
\hline Gambling & $3 \mathrm{rd}$ & Thilman, George & $4 / 1 / 1856$ & $\begin{array}{l}27-02-07-06 \mathrm{~F} . \\
44\end{array}$ \\
\hline Gambling & 2nd & $\begin{array}{l}\text { Thompson, } \\
\text { Jackson }\end{array}$ & $5 / 111 / 1857$ & $\begin{array}{l}27-02-07-06 \mathrm{~F} . \\
45\end{array}$ \\
\hline Gambling & 2nd & $\begin{array}{l}\text { Thompson, } \\
\text { James }\end{array}$ & $3 / 1857$ & $\begin{array}{l}27-02-07-06 \mathrm{~F} . \\
48\end{array}$ \\
\hline Gambling & 2nd & Tompson, James & $2 / 24 / 1859$ & $\begin{array}{l}27-02-07-06 \mathrm{~F} . \\
51\end{array}$ \\
\hline Gambling & 2nd & Vaughn, William & $9 / 10 / 1856$ & $\begin{array}{l}27-02-07-07 \mathrm{~F} . \\
26\end{array}$ \\
\hline Gambling & 2nd & $\begin{array}{l}\text { Wallace, } \\
\text { Milligan }\end{array}$ & $4 / 1 / 1856$ & $\begin{array}{l}27-02-08-01 \mathrm{~F} . \\
02\end{array}$ \\
\hline Gambling & 2nd & Wiggins, D. & $5 / 6 / 1857$ & $\begin{array}{l}27-02-08-01 \mathrm{~F} . \\
45\end{array}$ \\
\hline Gambling & 2nd & Winegardner & $4 / 1856$ & $\begin{array}{l}27-02-08-01 \mathrm{~F} . \\
71\end{array}$ \\
\hline Gambling & 2nd & Yager, William & $11 / 26 / 1856$ & $\begin{array}{l}27-02-08-01 \mathrm{~F} . \\
87\end{array}$ \\
\hline Gambling & 2nd & $\begin{array}{l}\text { Thompson, } \\
\text { James }\end{array}$ & $2 / 15 / 1857$ & $\begin{array}{l}27-02-07-06 \mathrm{~F} . \\
47\end{array}$ \\
\hline Gambling & 2nd & $\begin{array}{l}\text { Thompson, } \\
\text { James }\end{array}$ & $4 / 1 / 1857$ & $\begin{array}{l}27-02-07-06 \mathrm{~F} . \\
47\end{array}$ \\
\hline Gambling & $1 \mathrm{st}$ & $\begin{array}{l}\text { Thompson, } \\
\text { Orville }\end{array}$ & $4 / 1 / 1856$ & $\begin{array}{l}27-02-07-06 \mathrm{~F} . \\
54\end{array}$ \\
\hline Gaming & 2nd & $\begin{array}{l}\text { Bickerstaff, } \\
\text { Sanford }\end{array}$ & $8 / 10 / 1856$ & $\begin{array}{l}27-02-05-07 \mathrm{~F} . \\
02\end{array}$ \\
\hline Gaming & 2nd & Bunch & 1859 & $\begin{array}{l}27-02-05-07 \mathrm{~F} . \\
57\end{array}$ \\
\hline Gaming & 2nd & Clark, Jacob & $11 / 1856$ & $\begin{array}{l}27-02-06-01 \mathrm{~F} . \\
35\end{array}$ \\
\hline Gaming & 2nd & Clark, Osker & $10 / 9 / 1856$ & $\begin{array}{l}27-02-06-01 \mathrm{~F} . \\
37\end{array}$ \\
\hline Gaming & 2nd & Clarke, William & $10 / 9 / 1856$ & $\begin{array}{l}27-02-06-01 \mathrm{~F} . \\
39\end{array}$ \\
\hline Gaming & 2nd & Doyle, Daniel & $4 / 15 / 1857$ & $\begin{array}{l}27-02-06-03 \mathrm{~F} . \\
45\end{array}$ \\
\hline Gaming & $1 \mathrm{st}$ & Ewing, William & $10 / 10 / 1856$ & $\begin{array}{l}27-02-06-04 \mathrm{~F} . \\
15\end{array}$ \\
\hline Gaming & $1 \mathrm{st}$ & Hutchins, James & $11 / 7 / 1856$ & $\begin{array}{l}27-02-06-06 \mathrm{~F} . \\
41\end{array}$ \\
\hline Gaming & 2nd & Johnson, David & $5 / 4 / 1857$ & $\begin{array}{l}27-02-06-07 \mathrm{~F} . \\
05\end{array}$ \\
\hline Gaming & 2nd & Jones, Samuel & $2 / 15 / 1857$ & $\begin{array}{l}27-02-06-07 \mathrm{~F} . \\
18\end{array}$ \\
\hline Gaming & 2nd & Fleshman, Perry & $3 / 11 / 1858$ & $\begin{array}{l}27-02-06-04 \mathrm{~F} . \\
38\end{array}$ \\
\hline $\begin{array}{l}\text { Giving Liquor to } \\
\text { Indians }\end{array}$ & $3 \mathrm{rd}$ & Ballard, John & $9 / 11 / 1858$ & $\begin{array}{l}27-02-05-06 \mathrm{~F} . \\
35\end{array}$ \\
\hline
\end{tabular}




\begin{tabular}{|c|c|c|c|c|}
\hline $\begin{array}{l}\text { Giving Liquor to } \\
\text { Indians }\end{array}$ & $3 \mathrm{rd}$ & Ficer, Archibald & $8 / 1 / 1857$ & $\begin{array}{l}27-02-06-04 \mathrm{~F} . \\
29\end{array}$ \\
\hline $\begin{array}{l}\text { Giving Liquor to } \\
\text { Indians }\end{array}$ & $3 \mathrm{rd}$ & Kerr, James & $8 / 21 / 1858$ & $\begin{array}{l}27-02-06-07 \mathrm{~F} . \\
41\end{array}$ \\
\hline $\begin{array}{l}\text { Giving Liquor to } \\
\text { Indians }\end{array}$ & $3 \mathrm{rd}$ & Seymore, M.R. & $9 / 9 / 1857$ & $\begin{array}{l}27-02-07-05 \mathrm{~F} . \\
31\end{array}$ \\
\hline Grand Larceny & 2nd & Anglea, William & $4 / 1858$ & $\begin{array}{l}27-02-05-06 \mathrm{~F} . \\
16\end{array}$ \\
\hline Grand Larceny & 2nd & Bouton, W.W. & $9 / 5 / 1856$ & $\begin{array}{l}27-02-05-07 \mathrm{~F} . \\
19\end{array}$ \\
\hline Grand Larceny & $1 \mathrm{st}$ & Bowen, Daniel & $9 / 1858$ & $\begin{array}{l}27-02-05-07 \mathrm{~F} . \\
21\end{array}$ \\
\hline Grand Larceny & 2nd & $\begin{array}{l}\text { Brown, John \& } \\
\text { Broils, John \& } \\
\text { Masterson, } \\
\text { James }\end{array}$ & $9 / 21 / 1856$ & $\begin{array}{l}27-02-05-07 \mathrm{~F} . \\
41\end{array}$ \\
\hline Grand Larceny & 2nd & Brown, John Jr. & 1856 & $\begin{array}{l}27-02-05-07 \mathrm{~F} . \\
49\end{array}$ \\
\hline Grand Larceny & $3 \mathrm{rd}$ & $\begin{array}{l}\text { Caain, George \& } \\
\text { Tanksley, } \\
\text { Harrison }\end{array}$ & $7 / 20 / 1859$ & $\begin{array}{l}27-02-06-01 \mathrm{~F} . \\
22\end{array}$ \\
\hline Grand Larceny & 2nd & Calvins, Charles & $10 / 26 / 1857$ & $\begin{array}{l}27-02-06-01 \mathrm{~F} . \\
23\end{array}$ \\
\hline Grand Larceny & 2nd & Clark, George & $2 / 2 / 1857$ & $\begin{array}{l}27-02-06-01 \mathrm{~F} . \\
31\end{array}$ \\
\hline Grand Larceny & 2nd & Dudley, Guiford & $9 / 1 / 1856$ & $\begin{array}{l}27-02-06-03 \mathrm{~F} . \\
48\end{array}$ \\
\hline Grand Larceny & $1 \mathrm{st}$ & Fisher, Jacob & $11 / 29 / 1858$ & $\begin{array}{l}27-02-06-04 \mathrm{~F} . \\
33\end{array}$ \\
\hline Grand Larceny & 2nd & Frakes, Oliver & $5 / 1856$ & $\begin{array}{l}27-02-06-04 \mathrm{~F} . \\
47\end{array}$ \\
\hline Grand Larceny & 2nd & Fultron, James & $9 / 7 / 1856$ & $\begin{array}{l}27-02-06-04 \mathrm{~F} . \\
62\end{array}$ \\
\hline Grand Larceny & 2nd & Hay, Milton & $2 / 8 / 1858$ & $\begin{array}{l}27-02-06-05 \mathrm{~F} . \\
71\end{array}$ \\
\hline Grand Larceny & 2nd & $\begin{array}{l}\text { Hay, Milton \& } \\
\text { Duffield, Joseph }\end{array}$ & $5 / 2 / 1857$ & $\begin{array}{l}27-02-06-05 \mathrm{~F} . \\
70\end{array}$ \\
\hline Grand Larceny & 2nd & Howard, Cyrus & $11 / 15 / 1855$ & $\begin{array}{l}27-02-06-06 \mathrm{~F} . \\
28\end{array}$ \\
\hline Grand Larceny & 2nd & Jamison, A. & $11 / 20 / 1856$ & $\begin{array}{l}27-02-06-06 \mathrm{~F} . \\
52\end{array}$ \\
\hline Grand Larceny & 2nd & $\begin{array}{l}\text { Lewis, Dr. S. \& } \\
\text { Jordan, Dr. R. }\end{array}$ & $9 / 25 / 1856$ & $\begin{array}{l}27-02-07-01 \mathrm{~F} . \\
22\end{array}$ \\
\hline Grand Larceny & 2nd & Miller, A.J. & $5 / 8 / 1858$ & $\begin{array}{l}27-02-07-02 \mathrm{~F} . \\
05\end{array}$ \\
\hline Grand Larceny & $1 \mathrm{st}$ & Myers, Reuben & $7 / 20 / 1858$ & $\begin{array}{l}27-02-07-02 \mathrm{~F} . \\
63\end{array}$ \\
\hline Grand Larceny & $?$ & Myers, William & $3 / 8 / 1858$ & $\begin{array}{l}27-02-07-02 \mathrm{~F} . \\
64\end{array}$ \\
\hline Grand Larceny & 3rd & Oldham, James & $10 / 24 / 1857$ & $\begin{array}{l}27-02-07-03 \mathrm{~F} . \\
15\end{array}$ \\
\hline Grand Larceny & $3 \mathrm{rd}$ & $\begin{array}{l}\text { Rogers, Jackson } \\
\text { \& Mallet, } \\
\text { Sherman }\end{array}$ & $7 / 14 / 1859$ & $\begin{array}{l}27-02-07-04 \mathrm{~F} . \\
40\end{array}$ \\
\hline Grand Larceny & 2nd & Stowell, Martin & $9 / 5 / 1856$ & $\begin{array}{l}27-02-07-06 \mathrm{~F} . \\
22\end{array}$ \\
\hline
\end{tabular}




\begin{tabular}{|c|c|c|c|c|}
\hline Grand Larceny & 1 st & Titchworth, John & $3 / 18 / 1859$ & $\begin{array}{l}27-02-07-06 \mathrm{~F} . \\
60\end{array}$ \\
\hline Grand Larceny & 2nd & Twitchel, Hank & $11 / 20 / 1856$ & $\begin{array}{l}27-02-07-07 \mathrm{~F} . \\
13\end{array}$ \\
\hline Grand Larceny & $?$ & Way, William & $10 / 29 / 1857$ & $\begin{array}{l}27-02-08-01 \mathrm{~F} . \\
15\end{array}$ \\
\hline Grand Larceny & 2nd & Whitlock, James & $9 / 16 / 1856$ & $\begin{array}{l}27-02-08-01 \mathrm{~F} . \\
44\end{array}$ \\
\hline Grand Larceny & $1 \mathrm{st}$ & Willett, Morgan & $8 / 1 / 1857$ & $\begin{array}{l}27-02-08-01 \mathrm{~F} . \\
46\end{array}$ \\
\hline Grand Larceny & Jackson Cnty. & $\begin{array}{l}\text { William, } \\
\text { Whitton \& } \\
\text { William }\end{array}$ & $9 / 1858$ & $\begin{array}{l}27-02-08-01 \mathrm{~F} . \\
48\end{array}$ \\
\hline $\begin{array}{l}\text { Harboring a } \\
\text { Horse Thief }\end{array}$ & $3 \mathrm{rd}$ & Downing, Davis & $7 / 1 / 1858$ & $\begin{array}{l}27-02-06-03 \mathrm{~F} . \\
41\end{array}$ \\
\hline $\begin{array}{l}\text { Helping a } \\
\text { Fugitive }\end{array}$ & $?$ & Smith, Samuel & $1 / 17 / 1856$ & $\begin{array}{l}27-02-07-05 \mathrm{~F} . \\
57\end{array}$ \\
\hline $\begin{array}{l}\text { Helping a } \\
\text { Fugitive }\end{array}$ & $1 \mathrm{st}$ & Spenser, John & $6 / 28 / 1859$ & $\begin{array}{l}27-02-07-05 \mathrm{~F} . \\
65\end{array}$ \\
\hline Horse Larceny & ? & $\begin{array}{l}\text { Hanschen, } \\
\text { William }\end{array}$ & $9 / 1859$ & $\begin{array}{l}27-02-06-05 \mathrm{~F} . \\
50\end{array}$ \\
\hline Horse Stealing & $1 \mathrm{st}$ & $\begin{array}{l}\text { Berry, Henry \& } \\
\text { McLane, Alfred }\end{array}$ & $4 / 35 / 1860$ & $\begin{array}{l}27-02-05-06 \mathrm{~F} . \\
61\end{array}$ \\
\hline Horse Stealing & $3 \mathrm{rd}$ & Blythe, A.E. & $8 / 21 / 1858$ & $\begin{array}{l}27-02-05-07 \mathrm{~F} . \\
15\end{array}$ \\
\hline Horse Stealing & 3rd & $\begin{array}{l}\text { Blythe, A.E. \& } \\
\text { Taylor, Dr. T. }\end{array}$ & $5 / 20 / 1858$ & $\begin{array}{l}27-02-05-07 \mathrm{~F} . \\
14\end{array}$ \\
\hline horse Stealing & $1 \mathrm{st}$ & Coleman & 1856 & $\begin{array}{l}27-02-06-02 \mathrm{~F} . \\
09\end{array}$ \\
\hline Horse Stealing & 3 rd & Devlin, Patrick & $1 / 1 / 1860$ & $\begin{array}{l}27-02-06-03 \mathrm{~F} . \\
25\end{array}$ \\
\hline Horse Stealing & Jefferson Cnty. & Diggs, Charles & $7 / 24 / 1856$ & $\begin{array}{l}27-02-06-03 \mathrm{~F} . \\
28\end{array}$ \\
\hline Horse Stealing & 2nd & $\begin{array}{l}\text { Ferguson, } \\
\text { Thomas }\end{array}$ & $11 / 13 / 1856$ & $\begin{array}{l}27-02-06-04 \mathrm{~F} . \\
67\end{array}$ \\
\hline Horse Stealing & $?$ & Harland, John & $7 / 18 / 1858$ & $\begin{array}{l}27-02-06-05 \mathrm{~F} . \\
52\end{array}$ \\
\hline Horse Stealing & $1 \mathrm{st}$ & $\begin{array}{l}\text { Hazelting, } \\
\text { Dalton }\end{array}$ & 9/30/1856 & $\begin{array}{l}27-02-06-05 \mathrm{~F} . \\
67\end{array}$ \\
\hline Horse Stealing & $3 \mathrm{rd}$ & $\begin{array}{l}\text { Kenworthy, } \\
\text { Amos }\end{array}$ & $1 / 1 / 1860$ & $\begin{array}{l}27-02-06-07 \mathrm{~F} . \\
40\end{array}$ \\
\hline Horse Stealing & $3 \mathrm{rd}$ & Kirby, Abram & $12 / 20 / 1859$ & $\begin{array}{l}27-02-06-07 \mathrm{~F} . \\
57\end{array}$ \\
\hline Horse Stealing & Douglas Cnty. & Sanders & $7 / 3 / 1856$ & $\begin{array}{l}27-02-07-05 \mathrm{~F} . \\
09\end{array}$ \\
\hline Horse Stealing & 2nd & Schaffer, Joseph & $1 / 1 / 1858$ & $\begin{array}{l}27-02-07-05 \mathrm{~F} . \\
12\end{array}$ \\
\hline Horse Stealing & $3 \mathrm{rd}$ & $\begin{array}{l}\text { Shamblin, } \\
\text { Lafayette }\end{array}$ & $7 / 27 / 1858$ & $\begin{array}{l}27-02-07-05 \mathrm{~F} . \\
36\end{array}$ \\
\hline Horse Stealing & 3rd & Smith, Levi & $6 / 7 / 1859$ & $\begin{array}{l}27-02-07-05 \mathrm{~F} . \\
53\end{array}$ \\
\hline Horse Stealing & $3 \mathrm{rd}$ & Steel, Aleck & $1 / 1 / 1860$ & $\begin{array}{l}27-02-07-06 \mathrm{~F} . \\
06\end{array}$ \\
\hline Horse Stealing & $3 \mathrm{rd}$ & Steel, Emanuel & $1 / 1 / 1860$ & $\begin{array}{l}\text { 27-02-07-06 F. } \\
07\end{array}$ \\
\hline
\end{tabular}




\begin{tabular}{|c|c|c|c|c|}
\hline Horse Stealing & 3rd & Stinson, John & $1 / 1 / 1859$ & $\begin{array}{l}27-02-07-06 \mathrm{~F} . \\
16\end{array}$ \\
\hline Horse Stealing & $3 \mathrm{rd}$ & Street & $5 / 21 / 1860$ & $\begin{array}{l}27-02-07-06 \mathrm{~F} . \\
25\end{array}$ \\
\hline Horse Stealing & $1 \mathrm{st}$ & Titchworth, John & $7 / 30 / 1858$ & $\begin{array}{l}27-02-07-06 \mathrm{~F} . \\
58\end{array}$ \\
\hline Horse Stealing & 3 rd & Turner, Stephen & $1 / 1 / 1860$ & $\begin{array}{l}27-02-07-07 \mathrm{~F} . \\
11\end{array}$ \\
\hline Horse Stealing & 3rd & Waggoner, J.S. & $7 / 15 / 1858$ & $\begin{array}{l}27-02-07-07 \mathrm{~F} . \\
30\end{array}$ \\
\hline Horse Stealing & $1 \mathrm{st}$ & Titchworth, John & $3 / 25 / 1858$ & $\begin{array}{l}27-02-07-06 \mathrm{~F} . \\
58\end{array}$ \\
\hline $\begin{array}{l}\text { Horse Stealing } \\
\text { on Osage Indian } \\
\text { Lands }\end{array}$ & $3 \mathrm{rd}$ & Calloway, James & $5 / 17 / 1860$ & $\begin{array}{l}27-02-06-01 \mathrm{~F} . \\
24\end{array}$ \\
\hline $\begin{array}{l}\text { Horse Stealing } \\
\text { on Osage Indian } \\
\text { Lands }\end{array}$ & 3 rd & Calloway, Squire & $12 / 1 / 1858$ & $\begin{array}{l}27-02-06-01 \mathrm{~F} . \\
25\end{array}$ \\
\hline Illegal Detention & $?$ & $\begin{array}{l}\text { Wright, John \& } \\
\text { Lane, James }\end{array}$ & $9 / 31 / 1856$ & $\begin{array}{l}27-02-08-01 \mathrm{~F} . \\
85\end{array}$ \\
\hline Illegal Restraint & Douglas Cnty. & Lane, James & $8 / 28 / 1856$ & $\begin{array}{l}27-02-07-01 \mathrm{~F} . \\
06\end{array}$ \\
\hline $\begin{array}{l}\text { Introducing } \\
\text { Liquor into } \\
\text { Indian Country }\end{array}$ & 3rd & Ballard, John & $10 / 1 / 1857$ & $\begin{array}{l}27-02-05-06 \mathrm{~F} . \\
34\end{array}$ \\
\hline $\begin{array}{l}\text { Introducing } \\
\text { Liquor into } \\
\text { Indian country }\end{array}$ & $3 \mathrm{rd}$ & Ballard, Richard & $10 / 1858$ & $\begin{array}{l}27-02-05-06 \mathrm{~F} . \\
38\end{array}$ \\
\hline $\begin{array}{l}\text { Introducing } \\
\text { Liquor into } \\
\text { Indian Country }\end{array}$ & $3 \mathrm{rd}$ & Baxter, John & $6 / 1 / 1857$ & $\begin{array}{l}27-02-05-06 \mathrm{~F} . \\
52\end{array}$ \\
\hline $\begin{array}{l}\text { Introducing } \\
\text { Liquor into } \\
\text { Indian Country }\end{array}$ & 3rd & Carr, James & $10 / 1858$ & $\begin{array}{l}27-02-06-01 \mathrm{~F} . \\
10\end{array}$ \\
\hline $\begin{array}{l}\text { Introducing } \\
\text { Liquor into } \\
\text { Indian Country }\end{array}$ & 3rd & Glandon, Isaac & $12 / 20 / 1858$ & $\begin{array}{l}27-02-06-05 \mathrm{~F} . \\
23\end{array}$ \\
\hline $\begin{array}{l}\text { Introducing } \\
\text { Liquor into } \\
\text { Indian Country }\end{array}$ & $?$ & Harris, John & $6 / 21 / 1860$ & $\begin{array}{l}27-02-06-05 \mathrm{~F} . \\
56\end{array}$ \\
\hline Jury Excuse & 2nd & White, Thomas & $5 / 6 / 1859$ & $\begin{array}{l}27-02-08-01 \mathrm{~F} . \\
43\end{array}$ \\
\hline $\begin{array}{l}\text { Keeping a } \\
\text { Gambling House }\end{array}$ & $1 \mathrm{st}$ & $\begin{array}{l}\text { Blonnerhasset, } \\
\text { Thomas }\end{array}$ & $4 / 1858$ & $\begin{array}{l}27-02-06-07 \mathrm{~F} . \\
09\end{array}$ \\
\hline $\begin{array}{l}\text { Keeping a } \\
\text { Gambling House }\end{array}$ & $1 \mathrm{st}$ & Burke, Thomas & $8 / 1 / 1857$ & $\begin{array}{l}27-02-05-07 \mathrm{~F} . \\
60\end{array}$ \\
\hline $\begin{array}{l}\text { Keeping a } \\
\text { Gambling House }\end{array}$ & 2nd & $\begin{array}{l}\text { Douglass, } \\
\text { William }\end{array}$ & $1 / 12 / 1857$ & $\begin{array}{l}27-02-06-03 \mathrm{~F} . \\
40\end{array}$ \\
\hline $\begin{array}{l}\text { Keeping a } \\
\text { Gaming House }\end{array}$ & $1 \mathrm{st}$ & Partin, Martin & $9 / 1 / 1857$ & $\begin{array}{l}27-02-07-03 \mathrm{~F} . \\
32\end{array}$ \\
\hline $\begin{array}{l}\text { Keeping a } \\
\text { Gaming House }\end{array}$ & 2nd & Patty, Robert & $1 / 12 / 1857$ & $\begin{array}{l}27-02-07-03 \mathrm{~F} . \\
40\end{array}$ \\
\hline $\begin{array}{l}\text { Keeping a } \\
\text { Gaming House }\end{array}$ & $1 \mathrm{st}$ & $\begin{array}{l}\text { Reinhicule, } \\
\text { Fredrick }\end{array}$ & $9 / 1 / 1857$ & $\begin{array}{l}27-02-07-04 \mathrm{~F} . \\
16\end{array}$ \\
\hline $\begin{array}{l}\text { Keeping a } \\
\text { Gaming House }\end{array}$ & 2nd & $\begin{array}{l}\text { Spradling, } \\
\text { Obediah }\end{array}$ & $2 / 4 / 1858$ & $\begin{array}{l}27-02-07-05 \mathrm{~F} . \\
71\end{array}$ \\
\hline
\end{tabular}




\begin{tabular}{|c|c|c|c|c|}
\hline $\begin{array}{l}\text { Keeping a } \\
\text { Gaming Table }\end{array}$ & 2 nd & Dolye, James & $3 / 15 / 1857$ & $\begin{array}{l}27-02-06-03 \mathrm{~F} . \\
46\end{array}$ \\
\hline $\begin{array}{l}\text { Keeping a } \\
\text { Gaming Table }\end{array}$ & 2 nd & Doyle, James & $3 / 15 / 1857$ & $\begin{array}{l}27-02-06-03 \mathrm{~F} . \\
46\end{array}$ \\
\hline $\begin{array}{l}\text { Keeping a } \\
\text { Gaming Table }\end{array}$ & 2 nd & Doyle, James & $5 / 14 / 1857$ & $\begin{array}{l}27-02-06-03 \mathrm{~F} . \\
46\end{array}$ \\
\hline $\begin{array}{l}\text { Keeping a House } \\
\text { of Ill Fame }\end{array}$ & $1 \mathrm{st}$ & Dunbar, Henry & $4 / 4 / 1858$ & $\begin{array}{l}27-02-06-03 \mathrm{~F} . \\
54\end{array}$ \\
\hline $\begin{array}{l}\text { Keeping Grocery } \\
\text { Open on Sunday }\end{array}$ & 2 nd & Gearthart & $5 / 18 / 1856$ & $\begin{array}{l}27-02-06-05 \mathrm{~F} . \\
13\end{array}$ \\
\hline Kidnapping & Douglas Cnty. & Reid & $9 / 19 / 1856$ & $\begin{array}{l}27-02-07-04 \mathrm{~F} . \\
08\end{array}$ \\
\hline Killing a Horse & 2nd & $\begin{array}{l}\text { Moffet, Orlando } \\
\& \text { Bird, Harmon }\end{array}$ & $11 / 19 / 1855$ & $\begin{array}{l}27-02-07-02 \mathrm{~F} . \\
31\end{array}$ \\
\hline Larceny & Douglas Cnty. & $\begin{array}{l}\text { Brown \& Carter } \\
\text { \& Lenhart }\end{array}$ & $6 / 9 / 1856$ & $\begin{array}{l}27-02-05-07 \mathrm{~F} . \\
44\end{array}$ \\
\hline Larceny & 1st, 2nd & Cahee, Vincent & $10 / 1 / 1856$ & $\begin{array}{l}27-02-06-02 \mathrm{~F} . \\
04\end{array}$ \\
\hline Larceny & 3rd & $\begin{array}{l}\text { Cardwell, } \\
\text { George }\end{array}$ & $9 / 29 / 1859$ & $\begin{array}{l}27-02-06-01 \mathrm{~F} . \\
04\end{array}$ \\
\hline Larceny & 2nd & Clark, George & $7 / 10 / 1856$ & $\begin{array}{l}27-02-06-01 \mathrm{~F} . \\
32\end{array}$ \\
\hline Larceny & 3rd & Cox, C. & $8 / 21 / 1858$ & $\begin{array}{l}27-02-06-02 \mathrm{~F} . \\
23\end{array}$ \\
\hline Larceny & $1 \mathrm{st}$ & Davis, Isaac & 9/9/1856 & $\begin{array}{l}27-02-06-03 \mathrm{~F} . \\
11\end{array}$ \\
\hline Larceny & $1 \mathrm{st}$ & Day, Charles & $7 / 19 / 1856$ & $\begin{array}{l}27-02-06-03 \mathrm{~F} . \\
42\end{array}$ \\
\hline Larceny & 2nd & $\begin{array}{l}\text { Delaughter, } \\
\text { George \& Carter }\end{array}$ & $8 / 25 / 1856$ & $\begin{array}{l}27-02-06-03 \mathrm{~F} . \\
22\end{array}$ \\
\hline Larceny & $3 \mathrm{rd}$ & Devlin, Pat & $10 / 20 / 1859$ & $\begin{array}{l}27-02-06-03 \mathrm{~F} . \\
26\end{array}$ \\
\hline Larceny & 2 nd & $\begin{array}{l}\text { Duttfield, John } \\
\text { Elliot }\end{array}$ & $10 / 8 / 1857$ & $\begin{array}{l}27-02-06-03 \mathrm{~F} . \\
50\end{array}$ \\
\hline Larceny & $3 \mathrm{rd}$ & Fazec, Anthony & $5 / 21 / 1860$ & $\begin{array}{l}27-02-06-04 \mathrm{~F} . \\
26\end{array}$ \\
\hline Larceny & $1 \mathrm{st}$ & Gamble, Robert & $11 / 30 / 1857$ & $\begin{array}{l}27-02-06-05 \mathrm{~F} . \\
05\end{array}$ \\
\hline Larceny & 2nd & Halpain, Josiah & $9 / 5 / 1856$ & $\begin{array}{l}27-02-06-05 \mathrm{~F} . \\
45\end{array}$ \\
\hline Larceny & $1 \mathrm{st}$ & $\begin{array}{l}\text { Houston, } \\
\text { Adolphus }\end{array}$ & $6 / 1 / 1857$ & $\begin{array}{l}27-02-06-06 \mathrm{~F} . \\
25\end{array}$ \\
\hline Larceny & $1 \mathrm{st}$ & Ingerson, Henry & $9 / 10 / 1860$ & $\begin{array}{l}27-02-06-06 \mathrm{~F} . \\
49\end{array}$ \\
\hline Larceny & $3 \mathrm{rd}$ & $\begin{array}{l}\text { Jack (a large } \\
\text { Cherokee) }\end{array}$ & $7 / 10 / 1858$ & $\begin{array}{l}27-02-06-06 \mathrm{~F} . \\
51\end{array}$ \\
\hline Larceny & 2nd & $\begin{array}{l}\text { Kilbourn, Henry } \\
\text { \& Holmes, } \\
\text { Captain }\end{array}$ & $10 / 28 / 1856$ & $\begin{array}{l}27-02-06-07 \mathrm{~F} . \\
46\end{array}$ \\
\hline Larceny & 2nd & Mack, John & $9 / 8 / 1860$ & $\begin{array}{l}27-02-07-01 \mathrm{~F} . \\
45\end{array}$ \\
\hline Larceny & 2nd & $\begin{array}{l}\text { McCormick, } \\
\text { James }\end{array}$ & $5 / 1860$ & $\begin{array}{l}27-02-07-01 \mathrm{~F} . \\
60\end{array}$ \\
\hline Larceny & 3 rd & McGeehe, Asa & $1 / 1 / 1859$ & $\begin{array}{l}27-02-07-01 \mathrm{~F} . \\
77\end{array}$ \\
\hline
\end{tabular}




\begin{tabular}{|c|c|c|c|c|}
\hline Larceny & $3 \mathrm{rd}$ & McGinty, H. & $1 / 1 / 1859$ & $\begin{array}{l}27-02-07-01 \mathrm{~F} . \\
78\end{array}$ \\
\hline Larceny & $1 \mathrm{st}$ & McLane, Alfred & $9 / 10 / 1860$ & $\begin{array}{l}27-02-07-01 \mathrm{~F} . \\
85\end{array}$ \\
\hline Larceny & $1 \mathrm{st}$ & Smith, Clayton & $6 / 1 / 1857$ & $\begin{array}{l}27-02-07-05 \mathrm{~F} . \\
51\end{array}$ \\
\hline Larceny & 2 nd & Smith, Franklin & $7 / 15 / 1857$ & $\begin{array}{l}27-02-07-05 \mathrm{~F} . \\
52\end{array}$ \\
\hline Larceny & Douglas Cnty. & Stears, Lewis & $2 / 17 / 1858$ & $\begin{array}{l}27-02-07-06 \mathrm{~F} . \\
04\end{array}$ \\
\hline Larceny & Douglas Cnty. & Stowell, Martin & $9 / 3 / 1856$ & $\begin{array}{l}27-02-07-06 \mathrm{~F} . \\
23\end{array}$ \\
\hline Larceny & 2nd & Tolliwer, James & $9 / 23 / 1857$ & $\begin{array}{l}27-02-07-07 \mathrm{~F} . \\
02\end{array}$ \\
\hline Larceny & $1 \mathrm{st}$ & $\begin{array}{l}\text { Umberger, } \\
\text { George \& Davis, } \\
\text { Isaac \& Alderan, } \\
\text { Henry }\end{array}$ & $9 / 20 / 1856$ & $\begin{array}{l}27-02-07-07 \mathrm{~F} . \\
17\end{array}$ \\
\hline Larceny & $3 \mathrm{rd}$ & $\begin{array}{l}\text { Wells, } \\
\text { Washington }\end{array}$ & $11 / 11 / 1859$ & $\begin{array}{l}27-02-08-01 \mathrm{~F} . \\
27\end{array}$ \\
\hline Larceny & $1 \mathrm{st}$ & $\begin{array}{l}\text { Whipple, Charles } \\
\text { \& Jamison, John } \\
\text { A. }\end{array}$ & $9 / 9 / 1856$ & $\begin{array}{l}27-02-08-01 \mathrm{~F} . \\
38\end{array}$ \\
\hline Larceny & $1 \mathrm{st}$ & Wilson, David & $2 / 1 / 1860$ & $\begin{array}{l}27-02-08-01 \mathrm{~F} . \\
62\end{array}$ \\
\hline Larceny & 2nd & Wood, Samuel & $7 / 6 / 1857$ & $\begin{array}{l}27-02-08-01 \mathrm{~F} . \\
77\end{array}$ \\
\hline Larceny & 2nd & $\begin{array}{l}\text { Woolerber, } \\
\text { George }\end{array}$ & $2 / 18 / 1860$ & $\begin{array}{l}27-02-08-01 \mathrm{~F} . \\
81\end{array}$ \\
\hline Larceny & $?$ & $\begin{array}{l}\text { Baker, George \& } \\
\text { Beals, Walter }\end{array}$ & $9 / 18 / 56$ & $\begin{array}{l}27-02-05-06 \mathrm{~F} . \\
29\end{array}$ \\
\hline Larceny & $?$ & Banks, Amos & $3 / 27 / 1860$ & $\begin{array}{l}27-02-05-06 \mathrm{~F} . \\
39\end{array}$ \\
\hline Larceny & $1 \mathrm{st}$ & Ingerson, Henry & $1 / 10 / 1860$ & $\begin{array}{l}27-02-06-06 \mathrm{~F} . \\
48\end{array}$ \\
\hline Larceny & $1 \mathrm{st}$ & Kelley, William & $9 / 20 / 1858$ & $\begin{array}{l}27-02-06-07 \mathrm{~F} . \\
34\end{array}$ \\
\hline Larceny & $3 \mathrm{rd}$ & Levassey, Elan & $1 / 1 / 1859$ & $\begin{array}{l}27-02-07-01 \mathrm{~F} . \\
19\end{array}$ \\
\hline Larceny & 2nd & $\begin{array}{l}\text { Partridge, } \\
\text { William }\end{array}$ & $8 / 26 / 1856$ & $\begin{array}{l}27-02-07-03 \mathrm{~F} . \\
35\end{array}$ \\
\hline Larceny & $1 \mathrm{st}$ & Porter, William & $12 / 22 / 1856$ & $\begin{array}{l}27-02-07-03 \mathrm{~F} . \\
73\end{array}$ \\
\hline Larceny & 2nd & Stephens, John & $10 / 11 / 1856$ & $\begin{array}{l}27-02-07-06 \mathrm{~F} . \\
09\end{array}$ \\
\hline Larceny & $3 \mathrm{rd}$ & $\begin{array}{l}\text { Stornes, } \\
\text { Nicholas }\end{array}$ & $10 / 20 / 1859$ & $\begin{array}{l}27-02-07-06 \mathrm{~F} . \\
17\end{array}$ \\
\hline Larceny & $1 \mathrm{st}$ & Titchworth, John & $9 / 1858$ & $\begin{array}{l}27-02-07-06 \mathrm{~F} . \\
59\end{array}$ \\
\hline Larceny & $1 \mathrm{st}$ & $\begin{array}{l}\text { Umberger, } \\
\text { George \& Davis, } \\
\text { Isaac \& Alderan, } \\
\text { Henry }\end{array}$ & $10 / 11 / 1856$ & $\begin{array}{l}27-02-07-07 \mathrm{~F} . \\
17\end{array}$ \\
\hline Larceny & $1 \mathrm{st}$ & Wilson, John & $11 / 11 / 1856$ & $\begin{array}{l}27-02-08-01 \mathrm{~F} . \\
68\end{array}$ \\
\hline $\begin{array}{l}\text { Larceny of } \\
\text { Horses }\end{array}$ & 3rd & Freeblood & $5 / 21 / 1860$ & $\begin{array}{l}27-02-06-04 \mathrm{~F} . \\
50\end{array}$ \\
\hline
\end{tabular}




\begin{tabular}{|c|c|c|c|c|}
\hline $\begin{array}{l}\text { Larceny of } \\
\text { Indian Property }\end{array}$ & 3rd & Beeson, Jesse & $10 / 20 / 1859$ & $\begin{array}{l}27-02-05-06 \mathrm{~F} . \\
57\end{array}$ \\
\hline $\begin{array}{l}\text { Larceny of } \\
\text { Indian Property }\end{array}$ & $3 \mathrm{rd}$ & Haslett, John & $2 / 18 / 1860$ & $\begin{array}{l}27-02-06-05 \mathrm{~F} . \\
68\end{array}$ \\
\hline $\begin{array}{l}\text { Larceny of } \\
\text { Indian Property }\end{array}$ & $3 \mathrm{rd}$ & Marshal, Frank & $10 / 20 / 1859$ & $\begin{array}{l}27-02-07-01 \mathrm{~F} . \\
50\end{array}$ \\
\hline $\begin{array}{l}\text { Larceny of } \\
\text { Indian Property }\end{array}$ & $3 \mathrm{rd}$ & Moore, Richard & $10 / 29 / 1859$ & $\begin{array}{l}27-02-07-02 \mathrm{~F} . \\
40\end{array}$ \\
\hline $\begin{array}{l}\text { Larceny on } \\
\text { Indian Land }\end{array}$ & 3rd & Cain, John Riggs & $5 / 22 / 1860$ & $\begin{array}{l}27-02-06-01 \mathrm{~F} . \\
21\end{array}$ \\
\hline $\begin{array}{l}\text { Larceny on } \\
\text { Indian Land }\end{array}$ & $3 \mathrm{rd}$ & Gilworth, John & $10 / 20 / 1859$ & $\begin{array}{l}27-02-06-05 \mathrm{~F} . \\
22\end{array}$ \\
\hline $\begin{array}{l}\text { Larceny on } \\
\text { Indian Land }\end{array}$ & $3 \mathrm{rd}$ & Hale, E. & $11 / 28 / 1859$ & $\begin{array}{l}27-02-06-05 \mathrm{~F} . \\
44\end{array}$ \\
\hline $\begin{array}{l}\text { Larceny on } \\
\text { Indian Land }\end{array}$ & $3 \mathrm{rd}$ & Hazelton, John & $10 / 20 / 1859$ & $\begin{array}{l}27-02-06-05 \mathrm{~F} . \\
79\end{array}$ \\
\hline Maiming & 2nd & Day, John & $11 / 20 / 1854$ & $\begin{array}{l}27-02-06-03 \mathrm{~F} . \\
43\end{array}$ \\
\hline $\begin{array}{l}\text { Malicious } \\
\text { Mischief }\end{array}$ & 2nd & $\begin{array}{l}\text { Gleason, John \& } \\
\text { Henderson, John }\end{array}$ & $7 / 25 / 1857$ & $\begin{array}{l}27-02-06-05 \mathrm{~F} . \\
25\end{array}$ \\
\hline $\begin{array}{l}\text { Malicious } \\
\text { Mischief }\end{array}$ & 2nd & Hupp, Minor & $1 / 1 / 1856$ & $\begin{array}{l}27-02-06-06 \mathrm{~F} . \\
35\end{array}$ \\
\hline $\begin{array}{l}\text { Malicious } \\
\text { Mischief }\end{array}$ & 1st, 2nd & Hupp, Philip & $1 / 1 / 1856$ & $\begin{array}{l}27-02-06-06 \mathrm{~F} . \\
37\end{array}$ \\
\hline $\begin{array}{l}\text { Maliciously } \\
\text { Killing a Horse }\end{array}$ & 2nd & Bird, Harmon & $3 / 1856$ & $\begin{array}{l}27-02-05-07 \mathrm{~F} . \\
09\end{array}$ \\
\hline $\begin{array}{l}\text { Maliciously } \\
\text { Killing Two } \\
\text { Hogs }\end{array}$ & 2nd & $\begin{array}{l}\text { Cockran, } \\
\text { Benjamin }\end{array}$ & $2 / 1 / 1856$ & $\begin{array}{l}27-02-06-02 \mathrm{~F} . \\
01\end{array}$ \\
\hline $\begin{array}{l}\text { Motion to Stop } \\
\text { Indictment }\end{array}$ & $1 \mathrm{st}$ & Church, William & $9 / 19 / 1860$ & $\begin{array}{l}27-02-06-01 \mathrm{~F} . \\
20\end{array}$ \\
\hline Murder & 2nd & Adams, Edward & $8 / 11 / 1858$ & $\begin{array}{l}27-02-05-06 \mathrm{~F} . \\
05\end{array}$ \\
\hline Murder & 2nd & Adams, Edward & Spring, 1858 & $\begin{array}{l}27-02-05-06 \mathrm{~F} . \\
04\end{array}$ \\
\hline Murder & 2nd & Banfield, Mary & $12 / 19 / 1857$ & $\begin{array}{l}27-02-06-01 \mathrm{~F} . \\
02\end{array}$ \\
\hline Murder & $?$ & $\begin{array}{l}\text { Brown, John Sr. } \\
\text { \& Brown, Oliver } \\
\text { \& Brown, Owen } \\
\text { \& Brown, } \\
\text { Solomon }\end{array}$ & $5 / 24 / 1856$ & $\begin{array}{l}27-02-05-07 \mathrm{~F} . \\
42\end{array}$ \\
\hline Murder & 2nd & $\begin{array}{l}\text { Cansdell, Origin } \\
\text { \& Holmes, } \\
\text { Captain J.N. }\end{array}$ & $5 / 25 / 1857$ & $\begin{array}{l}27-02-06-01 \mathrm{~F} . \\
30\end{array}$ \\
\hline Murder & $1 \mathrm{st}$ & $\begin{array}{l}\text { Chapman, } \\
\text { Edward }\end{array}$ & $9 / 24 / 1856$ & $\begin{array}{l}27-02-06-01 \mathrm{~F} . \\
17\end{array}$ \\
\hline Murder & 2nd & $\begin{array}{l}\text { Clarke. G.W. \& } \\
\text { Burnes, N. }\end{array}$ & $8 / 20 / 1857$ & $\begin{array}{l}27-02-06-01 \mathrm{~F} . \\
33\end{array}$ \\
\hline Murder & $1 \mathrm{st}$ & Cleary, Jacob & $9 / 16 / 1856$ & $\begin{array}{l}27-02-06-01 \mathrm{~F} . \\
41\end{array}$ \\
\hline Murder & 2nd & Cushion, John & $12 / 9 / 1856$ & $\begin{array}{l}27-02-06-02 \mathrm{~F} . \\
45\end{array}$ \\
\hline Murder & 2nd & Funk, William & $2 / 21 / 1859$ & $\begin{array}{l}27-02-06-04 \mathrm{~F} . \\
66\end{array}$ \\
\hline Murder & 2rd & Goodell, John & $3 / 19 / 1860$ & $\begin{array}{l}27-02-06-05 \mathrm{~F} . \\
29\end{array}$ \\
\hline
\end{tabular}




\begin{tabular}{|c|c|c|c|c|}
\hline Murder & 2nd & Haggardy, Luke & $5 / 9 / 1859$ & $\begin{array}{l}27-02-06-05 \mathrm{~F} . \\
43\end{array}$ \\
\hline Murder & 2nd & Harrow, Charles & $2 / 20 / 1859$ & $\begin{array}{l}27-02-06-05 \mathrm{~F} . \\
58\end{array}$ \\
\hline Murder & 2nd & Hayes, Charles & $9 / 16 / 1856$ & $\begin{array}{l}27-02-06-05 \mathrm{~F} . \\
73\end{array}$ \\
\hline Murder & 2nd & Jones, John & $\begin{array}{l}5 / 1857 \\
\end{array}$ & $\begin{array}{l}27-02-06-07 \mathrm{~F} . \\
15\end{array}$ \\
\hline Murder & 2nd & Kibbee, Lucius & $12 / 29 / 1854$ & $\begin{array}{l}27-02-06-07 \mathrm{~F} . \\
45\end{array}$ \\
\hline Murder & $1 \mathrm{st}$ & Knighton, Amon & $7 / 31 / 1857$ & $\begin{array}{l}27-02-06-07 \mathrm{~F} . \\
59\end{array}$ \\
\hline Murder & 2nd & McGee, Jacob & $5 / 9 / 1857$ & $\begin{array}{l}27-02-07-01 \mathrm{~F} . \\
75\end{array}$ \\
\hline Murder & $1 \mathrm{st}$ & McVey, Francis & $12 / 16 / 1857$ & $\begin{array}{l}27-02-07-01 \mathrm{~F} . \\
91\end{array}$ \\
\hline Murder & $1 \mathrm{st}$ & Murphy, David & $9 / 28 / 1856$ & $\begin{array}{l}27-02-07-02 \mathrm{~F} . \\
54\end{array}$ \\
\hline Murder & $1 \mathrm{st}$ & Murphy, John & $10 / 12 / 1858$ & $\begin{array}{l}27-02-07-02 \mathrm{~F} . \\
56\end{array}$ \\
\hline Murder & $1 \mathrm{st}$ & Musser, George & $1 / 1 / 1858$ & $\begin{array}{l}27-02-07-02 \mathrm{~F} . \\
61\end{array}$ \\
\hline Murder & 2nd & Patrick, A.J. & $9 / 14 / 1856$ & $\begin{array}{l}27-02-07-03 \mathrm{~F} . \\
37\end{array}$ \\
\hline Murder & 3rd & Porter, William & $5 / 8 / 1858$ & $\begin{array}{l}27-02-07-03 \mathrm{~F} . \\
72\end{array}$ \\
\hline Murder & 2nd & $\begin{array}{l}\text { Right, William } \\
\text { \& Joy, John \& } \\
\text { Parker, Randall }\end{array}$ & $8 / 25 / 1858$ & $\begin{array}{l}27-02-07-04 \mathrm{~F} . \\
29\end{array}$ \\
\hline Murder & 1st, 2nd & Scott, Charlie & $7 / 17 / 1856$ & $\begin{array}{l}27-02-07-05 \mathrm{~F} . \\
17\end{array}$ \\
\hline Murder & Franklin Cnty. & Shirley, Thomas & $7 / 28 / 1858$ & $\begin{array}{l}27-02-07-05 \mathrm{~F} . \\
43\end{array}$ \\
\hline Murder & 2nd & $\begin{array}{l}\text { Shuler, Joseph \& } \\
\text { Deforest, Charles }\end{array}$ & $1 / 16 / 1858$ & $\begin{array}{l}27-02-07-05 \mathrm{~F} . \\
46\end{array}$ \\
\hline Murder & 2nd & Smith, Andrew & $9 / 16 / 1856$ & $\begin{array}{l}27-02-07-05 \mathrm{~F} . \\
49\end{array}$ \\
\hline Murder & Johnson Cnty. & $\begin{array}{l}\text { Surain, Bloom \& } \\
\text { Conatzer, Calvin }\end{array}$ & $8 / 6 / 1856$ & $\begin{array}{l}27-02-07-06 \mathrm{~F} . \\
29\end{array}$ \\
\hline Murder & 2nd & Sutton & $5 / 2 / 1857$ & $\begin{array}{l}27-02-07-06 \mathrm{~F} . \\
27\end{array}$ \\
\hline Murder & 2nd & Sutton, Isaac & $5 / 25 / 1857$ & $\begin{array}{l}27-02-07-06 \mathrm{~F} . \\
28\end{array}$ \\
\hline Murder & 1 st & $\begin{array}{l}\text { Thompson, } \\
\text { Orville }\end{array}$ & $4 / 22 / 1856$ & $\begin{array}{l}27-02-07-06 \mathrm{~F} . \\
55\end{array}$ \\
\hline Murder & 2nd & Townsley, James & $5 / 25 / 1856$ & $\begin{array}{l}27-02-07-07 \mathrm{~F} . \\
05\end{array}$ \\
\hline Murder & 2nd & Townsley, James & $5 / 25 / 1856$ & $\begin{array}{l}27-02-07-07 \mathrm{~F} . \\
06\end{array}$ \\
\hline Murder & 2nd & Townsley, James & $5 / 25 / 1856$ & $\begin{array}{l}27-02-07-07 \mathrm{~F} . \\
07\end{array}$ \\
\hline Murder & 2nd & Townsley, James & $5 / 25 / 1858$ & $\begin{array}{l}27-02-07-07 \mathrm{~F} . \\
04\end{array}$ \\
\hline Murder & $1 \mathrm{st}$ & Walker, Samuel & $9 / 16 / 1858$ & $\begin{array}{l}27-02-07-07 \mathrm{~F} . \\
37\end{array}$ \\
\hline
\end{tabular}




\begin{tabular}{|c|c|c|c|c|}
\hline Murder & 2nd & Walker, Samuel & $3 / 6 / 1858$ & $\begin{array}{l}27-02-07-07 \mathrm{~F} . \\
36\end{array}$ \\
\hline Murder & 2nd & Wells, William & $6 / 7 / 1858$ & $\begin{array}{l}27-02-08-01 \mathrm{~F} . \\
30\end{array}$ \\
\hline Murder & Bourbon Cnty. & Williams, Peyton & $5 / 17 / 1856$ & $\begin{array}{l}27-02-08-01 \mathrm{~F} . \\
56\end{array}$ \\
\hline Murder & 2nd & $\begin{array}{l}\text { Winegardner, } \\
\text { N.K. }\end{array}$ & $4 / 1 / 1856$ & $\begin{array}{l}27-02-08-01 \mathrm{~F} . \\
72\end{array}$ \\
\hline Murder & 2nd & Wright, Francis & $5 / 1857$ & $\begin{array}{l}27-02-08-01 \mathrm{~F} . \\
83\end{array}$ \\
\hline $\begin{array}{l}\text { Obstructing } \\
\text { Officers }\end{array}$ & 2nd & Gymeny, Frank & $12 / 1 / 1858$ & $\begin{array}{l}27-02-06-05 \mathrm{~F} . \\
42\end{array}$ \\
\hline $\begin{array}{l}\text { Obstructing } \\
\text { Officers }\end{array}$ & 2nd & $\begin{array}{l}\text { Tarmony, } \\
\text { Francis }\end{array}$ & $9 / 25 / 1858$ & $\begin{array}{l}27-02-07-07 \mathrm{~F} . \\
14\end{array}$ \\
\hline $\begin{array}{l}\text { Obtaining Goods } \\
\text { Under False } \\
\text { Pretenses }\end{array}$ & $3 \mathrm{rd}$ & $\begin{array}{l}\text { Waffle, Henry \& } \\
\text { Waffle, Douglas }\end{array}$ & $2 / 2 / 1858$ & $\begin{array}{l}27-02-07-07 \mathrm{~F} . \\
29\end{array}$ \\
\hline $\begin{array}{l}\text { Obtaining } \\
\text { Property Under } \\
\text { False Pretense }\end{array}$ & 2nd & Sims, Isaac & $3 / 19 / 1856$ & $\begin{array}{l}27-02-07-05 \mathrm{~F} . \\
34\end{array}$ \\
\hline $\begin{array}{l}\text { Office } \\
\text { Usurpation }\end{array}$ & 2nd & $\begin{array}{l}\text { Newson, } \\
\text { Benjamin }\end{array}$ & $5 / 1857$ & $\begin{array}{l}27-02-07-03 \mathrm{~F} . \\
05\end{array}$ \\
\hline $\begin{array}{l}\text { Office } \\
\text { Usurpation }\end{array}$ & $1 \mathrm{st}$ & Nixon, Joseph & $4 / 1 / 1856$ & $\begin{array}{l}27-02-07-03 \mathrm{~F} . \\
07\end{array}$ \\
\hline $\begin{array}{l}\text { Operating a } \\
\text { Ferry Without a } \\
\text { License }\end{array}$ & 2nd & Pappan, Lewis & $4 / 28 / 1857$ & $\begin{array}{l}27-02-07-03 \mathrm{~F} . \\
24\end{array}$ \\
\hline $\begin{array}{l}\text { Participation in } \\
\text { Topeka } \\
\text { Legislature }\end{array}$ & $?$ & Allen, Lyman & 1857 & $\begin{array}{l}27-02-05-06 \mathrm{~F} . \\
13\end{array}$ \\
\hline $\begin{array}{l}\text { Passing } \\
\text { Counterfeit } \\
\text { Money }\end{array}$ & $1 \mathrm{st}$ & Gilstein, James & $3 / 15 / 1860$ & $\begin{array}{l}27-02-06-05 \mathrm{~F} . \\
21\end{array}$ \\
\hline $\begin{array}{l}\text { Passing } \\
\text { Counterfeit } \\
\text { Money }\end{array}$ & $3 \mathrm{rd}$ & Taylor, Dr. J. & $1 / 1 / 1860$ & $\begin{array}{l}27-02-07-06 \mathrm{~F} . \\
40\end{array}$ \\
\hline Perjury & 2nd & Adams, Amos & $03 / 1859$ & $\begin{array}{l}27-02-05-06 \mathrm{~F} . \\
02\end{array}$ \\
\hline Perjury & 2nd & Burges, Henry & $6 / 1855$ & $\begin{array}{l}27-02-05-07 \mathrm{~F} . \\
59\end{array}$ \\
\hline Perjury & 2nd & Tompson, James & $5 / 3 / 1857$ & $\begin{array}{l}27-02-07-06 \mathrm{~F} . \\
49\end{array}$ \\
\hline Perjury & 2nd & Horne, Daniel & $3 / 30 / 1855$ & $\begin{array}{l}27-02-06-06 \mathrm{~F} . \\
22\end{array}$ \\
\hline $\begin{array}{l}\text { Pulling Down } \\
\text { and Carrying } \\
\text { Away a Cabin }\end{array}$ & 2nd & Cole, Joseph & $1 / 22 / 1857$ & $\begin{array}{l}27-02-06-02 \mathrm{~F} . \\
07\end{array}$ \\
\hline Rape & $1 \mathrm{st}$ & Jones, William & $4 / 10 / 1859$ & $\begin{array}{l}27-02-06-07 \mathrm{~F} . \\
20\end{array}$ \\
\hline Rebellion & $3 \mathrm{rd}$ & Brown, John & $10 / 24 / 1857$ & $\begin{array}{l}27-02-05-07 \mathrm{~F} . \\
47\end{array}$ \\
\hline Rebellion & $3 \mathrm{rd}$ & Curry, James & $10 / 24 / 1857$ & $\begin{array}{l}27-02-06-02 \mathrm{~F} . \\
42\end{array}$ \\
\hline Rebellion & 3rd & Davis, J. & $10 / 24 / 1856$ & $\begin{array}{l}27-02-06-03 \mathrm{~F} . \\
12\end{array}$ \\
\hline Rebellion & $3 \mathrm{rd}$ & Hoffnagle, J. & $10 / 24 / 1857$ & $\begin{array}{l}27-02-06-06 \mathrm{~F} . \\
14\end{array}$ \\
\hline
\end{tabular}




\begin{tabular}{|c|c|c|c|c|}
\hline $\begin{array}{l}\text { Receiving Stolen } \\
\text { Goods }\end{array}$ & Douglas Cnty. & Hinman, A.C. & $1 / 3 / 1856$ & $\begin{array}{l}27-02-06-06 \mathrm{~F} . \\
12\end{array}$ \\
\hline $\begin{array}{l}\text { Receiving Stolen } \\
\text { Goods }\end{array}$ & $3 \mathrm{rd}$ & Roby, Samuel & $11 / 8 / 1859$ & $\begin{array}{l}27-02-07-04 \mathrm{~F} . \\
38\end{array}$ \\
\hline $\begin{array}{l}\text { Receiving Stolen } \\
\text { Money }\end{array}$ & $3 \mathrm{rd}$ & Roby, Samuel & $11 / 14 / 1859$ & $\begin{array}{l}27-02-07-04 \mathrm{~F} . \\
38\end{array}$ \\
\hline $\begin{array}{l}\text { Receiving Stolen } \\
\text { Property }\end{array}$ & $3 \mathrm{rd}$ & Taylor, Dr. J. & $8 / 21 / 1858$ & $\begin{array}{l}27-02-07-06 \mathrm{~F} . \\
39\end{array}$ \\
\hline $\begin{array}{l}\text { Recovery of } \\
\text { Money }\end{array}$ & 2nd & Rodrigue, Ann & $11 / 20 / 1858$ & $\begin{array}{l}27-02-07-04 \mathrm{~F} . \\
41\end{array}$ \\
\hline $\begin{array}{l}\text { Rescuing a } \\
\text { Fugitive }\end{array}$ & 1st, 2nd & Hupp, Philip & $11 / 26 / 1855$ & $\begin{array}{l}27-02-06-06 \mathrm{~F} . \\
36\end{array}$ \\
\hline $\begin{array}{l}\text { Rescuing a } \\
\text { Prisoner }\end{array}$ & $1 \mathrm{st}$ & Donaldson, John & $11 / 6 / 1856$ & $\begin{array}{l}27-02-06-03 \mathrm{~F} . \\
31\end{array}$ \\
\hline $\begin{array}{l}\text { Rescuing a } \\
\text { Prisoner }\end{array}$ & 2nd & Richie, John & $10 / 6 / 1856$ & $\begin{array}{l}27-02-07-04 \mathrm{~F} . \\
24\end{array}$ \\
\hline $\begin{array}{l}\text { Resisting a U.S. } \\
\text { Officer }\end{array}$ & $3 \mathrm{rd}$ & Forbes, Robert & $8 / 7 / 1860$ & $\begin{array}{l}27-02-06-04 \mathrm{~F} . \\
44\end{array}$ \\
\hline $\begin{array}{l}\text { Resisting a U.S. } \\
\text { Officer }\end{array}$ & $3 \mathrm{rd}$ & Forbs, David & $10 / 5 / 1860$ & $\begin{array}{l}27-02-06-04 \mathrm{~F} . \\
41\end{array}$ \\
\hline $\begin{array}{l}\text { Resisting a U.S. } \\
\text { Officer }\end{array}$ & $3 \mathrm{rd}$ & Julien, Lewis & $8 / 7 / 1860$ & $\begin{array}{l}27-02-06-07 \mathrm{~F} . \\
21\end{array}$ \\
\hline $\begin{array}{l}\text { Resisting a U.S. } \\
\text { Officer }\end{array}$ & 2nd & $\begin{array}{l}\text { Lane, James \& } \\
\text { Crocker, J.G. }\end{array}$ & $2 / 15 / 1859$ & $\begin{array}{l}27-02-07-01 \mathrm{~F} . \\
09\end{array}$ \\
\hline Resisting Arrest & $3 \mathrm{rd}$ & Curry, James & $10 / 4 / 1860$ & $\begin{array}{l}27-02-06-02 \mathrm{~F} . \\
43\end{array}$ \\
\hline Resisting Arrest & 2nd & Jesse, William & $8 / 12 / 1857$ & $\begin{array}{l}27-02-06-06 \mathrm{~F} . \\
58\end{array}$ \\
\hline Resisting Arrest & Douglas Cnty. & $\begin{array}{l}\text { Sylverster, } \\
\text { Emery }\end{array}$ & $7 / 3 / 1858$ & $\begin{array}{l}27-02-07-06 \mathrm{~F} . \\
34\end{array}$ \\
\hline $\begin{array}{l}\text { Resisting } \\
\text { Execution of a } \\
\text { Writ }\end{array}$ & $?$ & $\begin{array}{l}\text { St. Paul, } \\
\text { Bertrand }\end{array}$ & $3 / 6 / 1858$ & $\begin{array}{l}27-02-07-05 \mathrm{~F} . \\
07\end{array}$ \\
\hline $\begin{array}{l}\text { Resisting } \\
\text { Officers }\end{array}$ & 2nd & Ritchie, John & $11 / 1 / 1859$ & $\begin{array}{l}27-02-07-04 \mathrm{~F} . \\
26\end{array}$ \\
\hline $\begin{array}{l}\text { Resisting U.S. } \\
\text { Marshal }\end{array}$ & 2nd & Ritchie, John & $3 / 19 / 1860$ & $\begin{array}{l}27-02-07-04 \mathrm{~F} . \\
27\end{array}$ \\
\hline $\begin{array}{l}\text { Resisting U.S. } \\
\text { Officer }\end{array}$ & 2nd & Reeder, Andrew & $4 / 1 / 1856$ & $\begin{array}{l}27-02-07-04 \mathrm{~F} . \\
12\end{array}$ \\
\hline Riot & 2nd & $\begin{array}{l}\text { Bluejacket, } \\
\text { Stephen }\end{array}$ & $9 / 12 / 1858$ & $\begin{array}{l}27-02-05-07 \mathrm{~F} . \\
13\end{array}$ \\
\hline Riot & $3 \mathrm{rd}$ & Farris, Sampson & $10 / 24 / 1857$ & $\begin{array}{l}27-02-06-04 \mathrm{~F} . \\
23\end{array}$ \\
\hline Riot & $3 \mathrm{rd}$ & Farriss, Daniel & $10 / 24 / 1857$ & $\begin{array}{l}27-02-06-04 \mathrm{~F} . \\
22\end{array}$ \\
\hline Riot & $3 \mathrm{rd}$ & Farriss, Richard & $10 / 24 / 1857$ & $\begin{array}{l}27-02-06-04 \mathrm{~F} . \\
22\end{array}$ \\
\hline Riot & 2nd & King, Mathew & $1 / 26 / 1858$ & $\begin{array}{l}27-02-06-07 \mathrm{~F} . \\
55\end{array}$ \\
\hline Riot & 2 nd & Martin, S.E. & $5 / 6 / 1857$ & $\begin{array}{l}27-02-07-01 \mathrm{~F} . \\
52\end{array}$ \\
\hline Riot & 2nd & Realf, Richard & $12 / 9 / 1856$ & $\begin{array}{l}27-02-07-04 \mathrm{~F} . \\
04\end{array}$ \\
\hline
\end{tabular}




\begin{tabular}{|c|c|c|c|c|}
\hline Riot & 1 st & $\begin{array}{l}\text { Reed, James \& } \\
\text { Long, General \& } \\
\text { Lucas, Corlew \& } \\
\text { McDaniel, BO \& } \\
\text { Sabstian, Alex \& } \\
\text { Sabastian, } \\
\text { Samuel }\end{array}$ & $2 / 26 / 1855$ & $\begin{array}{l}27-02-07-04 \mathrm{~F} . \\
10\end{array}$ \\
\hline Robbery & 1 st & Bainter, Ephraim & $10 / 13 / 1858$ & $\begin{array}{l}27-02-05-06 \mathrm{~F} . \\
28\end{array}$ \\
\hline Robbery & Douglas Cnty. & Buttan, Robert & $1 / 3 / 1856$ & $\begin{array}{l}27-02-05-07 \mathrm{~F} . \\
55\end{array}$ \\
\hline Robbery & Douglas Cnty. & Carpenter, Real & $10 / 4 / 1856$ & $\begin{array}{l}27-02-06-01 \mathrm{~F} . \\
09\end{array}$ \\
\hline Robbery & 2 nd & Cleary, John & $10 / 1856$ & $\begin{array}{l}27-02-06-01 \mathrm{~F} . \\
42\end{array}$ \\
\hline Robbery & 1st, 2nd & Crane, Hiram & $9 / 12 / 1856$ & $\begin{array}{l}27-02-06-02 \mathrm{~F} . \\
28\end{array}$ \\
\hline Robbery & 2nd & Curtis, William & $8 / 11 / 1858$ & $\begin{array}{l}27-02-06-02 \mathrm{~F} . \\
44\end{array}$ \\
\hline Robbery & 2nd & Dolman, Samuel & $9 / 26 / 1857$ & $\begin{array}{l}27-02-06-03 \mathrm{~F} . \\
29\end{array}$ \\
\hline Robbery & $1 \mathrm{st}$ & $\begin{array}{l}\text { Giddings, } \\
\text { Richard }\end{array}$ & $10 / 1 / 1856$ & $\begin{array}{l}27-02-06-05 \mathrm{~F} . \\
15\end{array}$ \\
\hline Robbery & 2nd & Graham, Samuel & $3 / 14 / 1860$ & $\begin{array}{l}27-02-06-05 \mathrm{~F} . \\
34\end{array}$ \\
\hline Robbery & Shawnee Cnty. & Harvey \& Moore & $9 / 1 / 1856$ & $\begin{array}{l}27-02-06-05 \mathrm{~F} . \\
65\end{array}$ \\
\hline Robbery & $1 \mathrm{st}$ & Harvey, James & $10 / 13 / 1856$ & $\begin{array}{l}27-02-06-05 \mathrm{~F} . \\
61\end{array}$ \\
\hline Robbery & $1 \mathrm{st}$ & Harvey, James & $11 / 28 / 1856$ & $\begin{array}{l}27-02-06-05 \mathrm{~F} . \\
64\end{array}$ \\
\hline Robbery & $1 \mathrm{st}$ & Harvey, James & $11 / 7 / 1856$ & $\begin{array}{l}27-02-06-05 \mathrm{~F} . \\
60\end{array}$ \\
\hline Robbery & $1 \mathrm{st}$ & Harvey, James & $11 / 7 / 1856$ & $\begin{array}{l}27-02-06-05 \mathrm{~F} . \\
62\end{array}$ \\
\hline Robbery & $1 \mathrm{st}$ & Harvey, James & $9 / 1 / 1856$ & $\begin{array}{l}27-02-06-05 \mathrm{~F} . \\
63\end{array}$ \\
\hline Robbery & $1 \mathrm{st}$ & Harvey, James & $9 / 9 / 1856$ & $\begin{array}{l}27-02-06-05 \mathrm{~F} . \\
63\end{array}$ \\
\hline Robbery & 2nd & Harvey, James & $12 / 23 / 1856$ & $\begin{array}{l}27-02-06-05 \mathrm{~F} . \\
63\end{array}$ \\
\hline Robbery & 2nd & Hayes, Homer & $9 / 15 / 1856$ & $\begin{array}{l}27-02-06-05 \mathrm{~F} . \\
75\end{array}$ \\
\hline Robbery & $1 \mathrm{st}$ & $\begin{array}{l}\text { Herrin, James \& } \\
\text { Unknown Party }\end{array}$ & $9 / 6 / 1856$ & $\begin{array}{l}27-02-07-07 \mathrm{~F} . \\
21\end{array}$ \\
\hline Robbery & $1 \mathrm{st}$ & Hurd, Jacob & $6 / 1 / 1856$ & $\begin{array}{l}27-02-06-06 \mathrm{~F} . \\
39\end{array}$ \\
\hline Robbery & $1 \mathrm{st}$ & $\begin{array}{l}\text { Jamieson, } \\
\text { Alexander }\end{array}$ & $9 / 8 / 1856$ & $\begin{array}{l}27-02-06-06 \mathrm{~F} . \\
53\end{array}$ \\
\hline Robbery & $1 \mathrm{st}$ & Jamieson, John & $11 / 17 / 1856$ & $\begin{array}{l}27-02-06-06 \mathrm{~F} . \\
54\end{array}$ \\
\hline Robbery & $1 \mathrm{st}$ & Kagle, John & $9 / 1856$ & $\begin{array}{l}27-02-06-07 \mathrm{~F} . \\
22\end{array}$ \\
\hline Robbery & $1 \mathrm{st}$ & Kemp, Thomas & $9 / 14 / 1856$ & $\begin{array}{l}27-02-06-07 \mathrm{~F} . \\
37\end{array}$ \\
\hline Robbery & $?$ & Kilburn, Henry & $10 / 28 / 1856$ & $\begin{array}{l}27-02-06-07 \mathrm{~F} . \\
48\end{array}$ \\
\hline
\end{tabular}




\begin{tabular}{|c|c|c|c|c|}
\hline Robbery & 2nd & Mason, Elihu & $2 / 27 / 1857$ & $\begin{array}{l}27-02-07-01 \mathrm{~F} . \\
54\end{array}$ \\
\hline Robbery & 2nd & Moffet, Charles & $9 / 3 / 1856$ & $\begin{array}{l}27-02-07-02 \mathrm{~F} . \\
26\end{array}$ \\
\hline Robbery & 2nd & Moffet, Charles & $9 / 4 / 1856$ & $\begin{array}{l}27-02-07-02 \mathrm{~F} . \\
27\end{array}$ \\
\hline Robbery & 2nd & Moffet, Charles & 9/4/1856 & $\begin{array}{l}27-02-07-02 \mathrm{~F} . \\
28\end{array}$ \\
\hline Robbery & 2nd & Moffit, Charles & $9 / 22 / 1856$ & $\begin{array}{l}27-02-07-02 \mathrm{~F} . \\
24\end{array}$ \\
\hline Robbery & $3 \mathrm{rd}$ & $\begin{array}{l}\text { Montgomery, } \\
\text { James }\end{array}$ & $12 / 16 / 1858$ & $\begin{array}{l}27-02-07-02 \mathrm{~F} . \\
34\end{array}$ \\
\hline Robbery & Douglas Cnty. & Power, George & $9 / 1 / 1856$ & $\begin{array}{l}27-02-07-03 \mathrm{~F} . \\
74\end{array}$ \\
\hline Robbery & 2nd & $\begin{array}{l}\text { Southern, } \\
\text { William }\end{array}$ & $8 / 11 / 1858$ & $\begin{array}{l}27-02-07-05 \mathrm{~F} . \\
69\end{array}$ \\
\hline Robbery & Douglas Cnty. & $\begin{array}{l}\text { Stearns, } \\
\text { Clarkson }\end{array}$ & $1 / 14 / 1856$ & $\begin{array}{l}27-02-07-06 \mathrm{~F} . \\
03\end{array}$ \\
\hline Robbery & 3rd & Steaurt, John & $12 / 13 / 1858$ & $\begin{array}{l}27-02-07-06 \mathrm{~F} . \\
05\end{array}$ \\
\hline Robbery & Douglas Cnty. & Stidham, Isaac & $9 / 24 / 1856$ & $\begin{array}{l}27-02-07-07 \mathrm{~F} . \\
19\end{array}$ \\
\hline Robbery & $3 \mathrm{rd}$ & Terrell & $12 / 13 / 1858$ & $\begin{array}{l}27-02-07-06 \mathrm{~F} . \\
43\end{array}$ \\
\hline Robbery & Douglas Cnty. & $\begin{array}{l}\text { Umberger \& Davis } \\
\& \text { Spradling }\end{array}$ & $9 / 7 / 1856$ & $\begin{array}{l}27-02-07-07 \mathrm{~F} . \\
18\end{array}$ \\
\hline Robbery & Douglas Cnty. & $\begin{array}{l}\text { Updegraft, } \\
\text { Edward }\end{array}$ & $9 / 22 / 1856$ & $\begin{array}{l}27-02-07-07 \mathrm{~F} . \\
23\end{array}$ \\
\hline Robbery & Douglas Cnty. & $\begin{array}{l}\text { Waitman, } \\
\text { William \& } \\
\text { Waitman, Henry }\end{array}$ & $9 / 26 / 1856$ & $\begin{array}{l}27-02-07-07 \mathrm{~F} . \\
32\end{array}$ \\
\hline Robbery & 2nd & Walker, Samuel & $9 / 15 / 1856$ & $\begin{array}{l}27-02-07-07 \mathrm{~F} . \\
39\end{array}$ \\
\hline Robbery & $3 \mathrm{rd}$ & Westfall, Dr. & $10 / 22 / 1856$ & $\begin{array}{l}27-02-08-01 \mathrm{~F} . \\
36\end{array}$ \\
\hline Robbery & 2nd & $\begin{array}{l}\text { White, Andrew } \\
\& \text { Curtin, Alfred }\end{array}$ & $9 / 15 / 1856$ & $\begin{array}{l}27-02-08-01 \mathrm{~F} . \\
39\end{array}$ \\
\hline $\begin{array}{l}\text { Robbery and } \\
\text { Concealment of } \\
\text { Stolen Goods }\end{array}$ & 2nd & Swain, Joseph & $2 / 8 / 1857$ & $\begin{array}{l}27-02-07-06 \mathrm{~F} . \\
30\end{array}$ \\
\hline $\begin{array}{l}\text { Robbery and } \\
\text { Concealment of } \\
\text { Stolen Goods }\end{array}$ & 2nd & Whipple, Charles & $10 / 1 / 1856$ & $\begin{array}{l}27-02-08-01 \mathrm{~F} . \\
37\end{array}$ \\
\hline $\begin{array}{l}\text { Robbing U.S. } \\
\text { Mail }\end{array}$ & 1st, 2nd & Crane, Hiram & $9 / 12 / 1856$ & $\begin{array}{l}27-02-06-02 \mathrm{~F} . \\
29\end{array}$ \\
\hline $\begin{array}{l}\text { Robbing U.S. } \\
\text { Mail }\end{array}$ & 3 rd & Dare, Benjamin & $4 / 1 / 1859$ & $\begin{array}{l}27-02-06-03 \mathrm{~F} . \\
07\end{array}$ \\
\hline $\begin{array}{l}\text { Robbing U.S. } \\
\text { Mail }\end{array}$ & 2nd & $\begin{array}{l}\text { Montgomery, } \\
\text { James }\end{array}$ & $7 / 29 / 1859$ & $\begin{array}{l}27-02-07-02 \mathrm{~F} . \\
35\end{array}$ \\
\hline $\begin{array}{l}\text { Robbing U.S. } \\
\text { Mail }\end{array}$ & $1 \mathrm{st}$ & $\begin{array}{l}\text { Richie, John \& } \\
\text { Kagle, John \& } \\
\text { Whipple, Charles }\end{array}$ & $9 / 11 / 1856$ & $\begin{array}{l}27-02-07-04 \mathrm{~F} . \\
25\end{array}$ \\
\hline Roulette & 2nd & Stewart, Owen & $1 / 1857$ & $\begin{array}{l}27-02-07-06 \mathrm{~F} . \\
14\end{array}$ \\
\hline $\begin{array}{l}\text { Secreting Stolen } \\
\text { Property }\end{array}$ & 3rd & Farris, Sampson & $10 / 24 / 1857$ & $\begin{array}{l}27-02-06-04 \mathrm{~F} . \\
24\end{array}$ \\
\hline
\end{tabular}




\begin{tabular}{|c|c|c|c|c|}
\hline Selling Liquor & $1 \mathrm{st}$ & Fahey, Tomas & $4 / 20 / 1857$ & $\begin{array}{l}27-02-06-04 \mathrm{~F} . \\
16\end{array}$ \\
\hline $\begin{array}{l}\text { Selling Liquor in } \\
\text { Indian Country }\end{array}$ & 2nd & McCune & $9 / 29 / 1860$ & $\begin{array}{l}27-02-07-01 \mathrm{~F} . \\
66\end{array}$ \\
\hline $\begin{array}{l}\text { Selling Liquor in } \\
\text { Indian Country }\end{array}$ & $3 \mathrm{rd}$ & Taylor, Martin & $10 / 1 / 1857$ & $\begin{array}{l}27-02-07-06 \mathrm{~F} . \\
38\end{array}$ \\
\hline $\begin{array}{l}\text { Selling Liquor } \\
\text { on Sunday }\end{array}$ & $1 \mathrm{st}$ & Burke, Thomas & $9 / 1857$ & $\begin{array}{l}27-02-05-07 \mathrm{~F} . \\
61\end{array}$ \\
\hline $\begin{array}{l}\text { Selling Liquor } \\
\text { on Sunday }\end{array}$ & $1 \mathrm{st}$ & $\begin{array}{l}\text { Deacon, } \\
\text { Gustavus }\end{array}$ & $9 / 25 / 1858$ & $\begin{array}{l}27-02-06-03 \mathrm{~F} . \\
19\end{array}$ \\
\hline $\begin{array}{l}\text { Selling Liquor } \\
\text { on Sunday }\end{array}$ & $1 \mathrm{st}$ & Horst, Herman & $4 / 25 / 1858$ & $\begin{array}{l}27-02-06-06 \mathrm{~F} . \\
23\end{array}$ \\
\hline $\begin{array}{l}\text { Selling Liquor } \\
\text { on Sunday }\end{array}$ & $1 \mathrm{st}$ & Horst. Herman & $9 / 2 / 1858$ & $\begin{array}{l}27-02-06-06 \mathrm{~F} . \\
23\end{array}$ \\
\hline $\begin{array}{l}\text { Selling Liquor } \\
\text { on Sunday }\end{array}$ & 2nd & Mitchel, John & $3 / 2 / 1856$ & $\begin{array}{l}27-02-07-02 \mathrm{~F} . \\
23\end{array}$ \\
\hline $\begin{array}{l}\text { Selling Liquor } \\
\text { on Sunday }\end{array}$ & $1 \mathrm{st}$ & Muer, James & $9 / 20 / 1857$ & $\begin{array}{l}27-02-07-02 \mathrm{~F} . \\
51\end{array}$ \\
\hline $\begin{array}{l}\text { Selling Liquor } \\
\text { on Sunday }\end{array}$ & $1 \mathrm{st}$ & Partin, Martin & $8 / 25 / 1858$ & $\begin{array}{l}27-02-07-03 \mathrm{~F} . \\
31\end{array}$ \\
\hline $\begin{array}{l}\text { Selling Liquor } \\
\text { on Sunday }\end{array}$ & $1 \mathrm{st}$ & $\begin{array}{l}\text { Reinhickle, } \\
\text { Fredrick }\end{array}$ & $9 / 6 / 1857$ & $\begin{array}{l}27-02-07-04 \mathrm{~F} . \\
17\end{array}$ \\
\hline $\begin{array}{l}\text { Selling Liquor } \\
\text { on Sunday }\end{array}$ & 2nd & $\begin{array}{l}\text { Stormes, } \\
\text { Nicholas }\end{array}$ & $5 / 11 / 1856$ & $\begin{array}{l}27-02-07-06 \mathrm{~F} . \\
18\end{array}$ \\
\hline $\begin{array}{l}\text { Selling Liquor } \\
\text { on Sunday }\end{array}$ & 2nd & $\begin{array}{l}\text { Thompson, } \\
\text { Jackson }\end{array}$ & $2 / 20 / 1857$ & $\begin{array}{l}27-02-07-06 \mathrm{~F} . \\
46\end{array}$ \\
\hline $\begin{array}{l}\text { Selling Liquor } \\
\text { on Sunday }\end{array}$ & 2nd & $\begin{array}{l}\text { Thompson, } \\
\text { James }\end{array}$ & $4 / 15 / 1857$ & $\begin{array}{l}27-02-07-06 \mathrm{~F} . \\
50\end{array}$ \\
\hline $\begin{array}{l}\text { Selling Liquor } \\
\text { on Sunday }\end{array}$ & $1 \mathrm{st}$ & $\begin{array}{l}\text { Tzattskowski, } \\
\text { Alexander }\end{array}$ & $4 / 25 / 1858$ & $\begin{array}{l}27-02-07-07 \mathrm{~F} . \\
16\end{array}$ \\
\hline $\begin{array}{l}\text { Selling Liquor to } \\
\text { Indians }\end{array}$ & $3 \mathrm{rd}$ & Bass, Peter & $11 / 1 / 1859$ & $\begin{array}{l}27-02-05-06 \mathrm{~F} . \\
46\end{array}$ \\
\hline $\begin{array}{l}\text { Selling Liquor to } \\
\text { Indians }\end{array}$ & $3 \mathrm{rd}$ & Baxter, John & $6 / 1 / 1857$ & $\begin{array}{l}27-02-05-06 \mathrm{~F} . \\
51\end{array}$ \\
\hline $\begin{array}{l}\text { Selling Liquor to } \\
\text { Indians }\end{array}$ & $1 \mathrm{st}$ & Bertrand, Amar & $6 / 25 / 1859$ & $\begin{array}{l}27-02-05-06 \mathrm{~F} . \\
63\end{array}$ \\
\hline $\begin{array}{l}\text { Selling Liquor to } \\
\text { Indians }\end{array}$ & 2nd & $\begin{array}{l}\text { Bickerstaff, } \\
\text { Sanford }\end{array}$ & $10 / 10 / 1856$ & $\begin{array}{l}27-02-05-07 \mathrm{~F} . \\
05\end{array}$ \\
\hline $\begin{array}{l}\text { Selling Liquor to } \\
\text { Indians }\end{array}$ & 2nd & $\begin{array}{l}\text { Bickerstaff, } \\
\text { Sanford }\end{array}$ & $10 / 20 / 1856$ & $\begin{array}{l}27-02-05-07 \mathrm{~F} . \\
05\end{array}$ \\
\hline $\begin{array}{l}\text { Selling Liquor to } \\
\text { Indians }\end{array}$ & 2nd & $\begin{array}{l}\text { Bickerstaff, } \\
\text { Sanford }\end{array}$ & $9 / 20 / 1856$ & $\begin{array}{l}27-02-05-07 \mathrm{~F} . \\
04\end{array}$ \\
\hline $\begin{array}{l}\text { Selling Liquor to } \\
\text { Indians }\end{array}$ & 2nd & Bien, Lewis & $2 / 29 / 1860$ & $\begin{array}{l}27-02-05-07 \mathrm{~F} . \\
06\end{array}$ \\
\hline $\begin{array}{l}\text { Selling Liquor to } \\
\text { Indians }\end{array}$ & $3 \mathrm{rd}$ & Bitterman & $11 / 12 / 1859$ & $\begin{array}{l}27-02-05-07 \mathrm{~F} . \\
11\end{array}$ \\
\hline $\begin{array}{l}\text { Selling Liquor to } \\
\text { Indians }\end{array}$ & 2nd & Bookey, George & $11 / 1 / 1859$ & $\begin{array}{l}27-02-05-07 \mathrm{~F} . \\
17\end{array}$ \\
\hline $\begin{array}{l}\text { Selling Liquor to } \\
\text { Indians }\end{array}$ & 1st, 2nd & Bruno, John & $10 / 29 / 1856$ & $\begin{array}{l}27-02-05-07 \mathrm{~F} . \\
53\end{array}$ \\
\hline $\begin{array}{l}\text { Selling Liquor to } \\
\text { Indians }\end{array}$ & 2nd & Buhler, Fredrick & $4 / 30 / 1860$ & $\begin{array}{l}27-02-05-07 \mathrm{~F} . \\
56\end{array}$ \\
\hline $\begin{array}{l}\text { Selling Liquor to } \\
\text { Indians }\end{array}$ & 1 st & $\begin{array}{l}\text { Deacon, } \\
\text { Gustavus }\end{array}$ & $9 / 14 / 1857$ & $\begin{array}{l}27-02-06-03 \mathrm{~F} . \\
18\end{array}$ \\
\hline $\begin{array}{l}\text { Selling Liquor to } \\
\text { Indians }\end{array}$ & $1 \mathrm{st}$ & Dean, Charles & $1 / 2 / 1858$ & $\begin{array}{l}27-02-06-03 \mathrm{~F} . \\
20\end{array}$ \\
\hline
\end{tabular}




\begin{tabular}{|c|c|c|c|c|}
\hline $\begin{array}{l}\text { Selling Liquor to } \\
\text { Indians }\end{array}$ & 2nd & $\begin{array}{l}\text { Donivan, } \\
\text { Timothy }\end{array}$ & $2 / 1855$ & $\begin{array}{l}27-02-06-03 \mathrm{~F} . \\
32\end{array}$ \\
\hline $\begin{array}{l}\text { Selling Liquor to } \\
\text { Indians }\end{array}$ & 2nd & $\begin{array}{l}\text { Donovan, } \\
\text { Thomas \& } \\
\text { Culbert, Michael }\end{array}$ & $2 / 18 / 1855$ & $\begin{array}{l}27-02-06-03 \mathrm{~F} . \\
35\end{array}$ \\
\hline $\begin{array}{l}\text { Selling Liquor to } \\
\text { Indians }\end{array}$ & 3rd & $\begin{array}{l}\text { Douglass, } \\
\text { Carlton }\end{array}$ & $11 / 20 / 1859$ & $\begin{array}{l}27-02-06-03 \mathrm{~F} . \\
38\end{array}$ \\
\hline $\begin{array}{l}\text { Selling Liquor to } \\
\text { Indians }\end{array}$ & $3 \mathrm{rd}$ & $\begin{array}{l}\text { Douglass. } \\
\text { Tarlton }\end{array}$ & $1 / 1 / 1860$ & $\begin{array}{l}27-02-06-03 \mathrm{~F} . \\
39\end{array}$ \\
\hline $\begin{array}{l}\text { Selling Liquor to } \\
\text { Indians }\end{array}$ & $1 \mathrm{st}$ & $\begin{array}{l}\text { Fishmaker, } \\
\text { Charles }\end{array}$ & $4 / 1 / 1856$ & $\begin{array}{l}27-02-06-04 \mathrm{~F} . \\
34\end{array}$ \\
\hline $\begin{array}{l}\text { Selling Liquor to } \\
\text { Indians }\end{array}$ & 2nd & French, James & $11 / 12 / 1859$ & $\begin{array}{l}27-02-06-04 \mathrm{~F} . \\
52\end{array}$ \\
\hline $\begin{array}{l}\text { Selling Liquor to } \\
\text { Indians }\end{array}$ & 2nd & Fry, Samuel & $3 / 31 / 1858$ & $\begin{array}{l}27-02-06-04 \mathrm{~F} . \\
54\end{array}$ \\
\hline $\begin{array}{l}\text { Selling Liquor to } \\
\text { Indians }\end{array}$ & 1st, 2nd & Fuller. James & $4 / 1 / 1856$ & $\begin{array}{l}27-02-06-04 \mathrm{~F} . \\
58\end{array}$ \\
\hline $\begin{array}{l}\text { Selling Liquor to } \\
\text { Indians }\end{array}$ & 2nd & Gearhart & $12 / 2 / 1855$ & $\begin{array}{l}27-02-06-05 \mathrm{~F} . \\
12\end{array}$ \\
\hline $\begin{array}{l}\text { Selling Liquor to } \\
\text { Indians }\end{array}$ & 3rd & Goldstein & $12 / 1 / 1858$ & $\begin{array}{l}27-02-06-05 \mathrm{~F} . \\
28\end{array}$ \\
\hline $\begin{array}{l}\text { Selling Liquor to } \\
\text { Indians }\end{array}$ & 3rd & $\begin{array}{l}\text { Goodman, } \\
\text { William }\end{array}$ & $11 / 1 / 1859$ & $\begin{array}{l}27-02-06-05 \mathrm{~F} . \\
31\end{array}$ \\
\hline $\begin{array}{l}\text { Selling Liquor to } \\
\text { Indians }\end{array}$ & $1 \mathrm{st}$ & Hunt & $9 / 14 / 1857$ & $\begin{array}{l}27-02-06-06 \mathrm{~F} . \\
32\end{array}$ \\
\hline $\begin{array}{l}\text { Selling Liquor to } \\
\text { Indians }\end{array}$ & $3 \mathrm{rd}$ & Killebrew, Elias & $7 / 30 / 1858$ & $\begin{array}{l}27-02-06-07 \mathrm{~F} . \\
50\end{array}$ \\
\hline $\begin{array}{l}\text { Selling Liquor to } \\
\text { Indians }\end{array}$ & 3rd & $\begin{array}{l}\text { Killebrew, } \\
\text { Pinkney }\end{array}$ & $6 / 15 / 1857$ & $\begin{array}{l}27-02-06-07 \mathrm{~F} . \\
51\end{array}$ \\
\hline $\begin{array}{l}\text { Selling Liquor to } \\
\text { Indians }\end{array}$ & 2nd & King, Nelson & $1 / 1 / 1856$ & $\begin{array}{l}27-02-06-07 \mathrm{~F} . \\
56\end{array}$ \\
\hline $\begin{array}{l}\text { Selling Liquor to } \\
\text { Indians }\end{array}$ & 3rd & Laberty, Peter & $11 / 1 / / 1859$ & $\begin{array}{l}27-02-07-01 \mathrm{~F} . \\
01\end{array}$ \\
\hline $\begin{array}{l}\text { Selling Liquor to } \\
\text { Indians }\end{array}$ & 2nd & $\begin{array}{l}\text { McKnown, } \\
\text { Robert }\end{array}$ & $2 / 1 / 1857$ & $\begin{array}{l}27-02-07-01 \mathrm{~F} . \\
84\end{array}$ \\
\hline $\begin{array}{l}\text { Selling Liquor to } \\
\text { Indians }\end{array}$ & 2nd & Milne, David & $12 / 19 / 1857$ & $\begin{array}{l}27-02-07-02 \mathrm{~F} . \\
19\end{array}$ \\
\hline $\begin{array}{l}\text { Selling Liquor to } \\
\text { Indians }\end{array}$ & 2nd & Mullen, Glad & $4 / 1 / 1856$ & $\begin{array}{l}27-02-07-02 \mathrm{~F} . \\
53\end{array}$ \\
\hline $\begin{array}{l}\text { Selling Liquor to } \\
\text { Indians }\end{array}$ & 2nd & Murry, Thomas & $9 / 12 / 1856$ & $\begin{array}{l}27-02-07-02 \mathrm{~F} . \\
60\end{array}$ \\
\hline $\begin{array}{l}\text { Selling Liquor to } \\
\text { Indians }\end{array}$ & $2 \mathrm{st}$ & Person, Nersin & $4 / 10 / 1858$ & $\begin{array}{l}27-02-07-03 \mathrm{~F} . \\
47\end{array}$ \\
\hline $\begin{array}{l}\text { Selling Liquor to } \\
\text { Indians }\end{array}$ & 3rd & Phillips, James & $10 / 12 / 1858$ & $\begin{array}{l}27-02-07-03 \mathrm{~F} . \\
58\end{array}$ \\
\hline $\begin{array}{l}\text { Selling Liquor to } \\
\text { Indians }\end{array}$ & 3rd & Pinnco & $11 / 1 / 1859$ & $\begin{array}{l}27-02-07-03 \mathrm{~F} . \\
63\end{array}$ \\
\hline $\begin{array}{l}\text { Selling Liquor to } \\
\text { Indians }\end{array}$ & 2nd & Red, D. & $3 / 17 / 1856$ & $\begin{array}{l}27-02-07-04 \mathrm{~F} . \\
06\end{array}$ \\
\hline $\begin{array}{l}\text { Selling Liquor to } \\
\text { Indians }\end{array}$ & 3rd & Scott, James & $11 / 10 / 1859$ & $\begin{array}{l}27-02-07-05 \mathrm{~F} . \\
18\end{array}$ \\
\hline $\begin{array}{l}\text { Selling Liquor to } \\
\text { Indians }\end{array}$ & $3 \mathrm{rd}$ & Scott, William & $11 / 20 / 1859$ & $\begin{array}{l}27-02-07-05 \mathrm{~F} . \\
23\end{array}$ \\
\hline $\begin{array}{l}\text { Selling Liquor to } \\
\text { Indians }\end{array}$ & $3 \mathrm{rd}$ & $\begin{array}{l}\text { Southern, } \\
\text { William }\end{array}$ & $1 / 1 / 1860$ & $\begin{array}{l}27-02-07-05 \mathrm{~F} . \\
63\end{array}$ \\
\hline
\end{tabular}




\begin{tabular}{|c|c|c|c|c|}
\hline $\begin{array}{l}\text { Selling Liquor to } \\
\text { Indians }\end{array}$ & 3rd & $\begin{array}{l}\text { Trobinger, } \\
\text { William }\end{array}$ & $8 / 1 / 1858$ & $\begin{array}{l}\text { 27-02-07-07 F. } \\
08\end{array}$ \\
\hline $\begin{array}{l}\text { Selling Liquor to } \\
\text { Indians }\end{array}$ & $3 \mathrm{rd}$ & Wakefield, N.R. & $12 / 2 / 1859$ & $\begin{array}{l}27-02-07-07 \mathrm{~F} . \\
33\end{array}$ \\
\hline $\begin{array}{l}\text { Selling Liquor to } \\
\text { Indians }\end{array}$ & $?$ & $\begin{array}{l}\text { Harris, } \\
\text { Covington }\end{array}$ & $9 / 30 / 1858$ & $\begin{array}{l}27-02-06-05 \mathrm{~F} . \\
55\end{array}$ \\
\hline $\begin{array}{l}\text { Selling Liquor to } \\
\text { Indians }\end{array}$ & $1 \mathrm{st}$ & Melon, Cobb & $1 / 2 / 1859$ & $\begin{array}{l}27-02-07-02 \mathrm{~F} . \\
01\end{array}$ \\
\hline $\begin{array}{l}\text { Selling Liquor to } \\
\text { Indians }\end{array}$ & $1 \mathrm{st}$ & Milne, David & $4 / 2 / 1856$ & $\begin{array}{l}27-02-07-02 \mathrm{~F} . \\
17\end{array}$ \\
\hline $\begin{array}{l}\text { Selling Liquor to } \\
\text { Indians }\end{array}$ & 2nd & Moody, Oliver & $5 / 20 / 1860$ & $\begin{array}{l}27-02-07-02 \mathrm{~F} . \\
37\end{array}$ \\
\hline $\begin{array}{l}\text { Selling Liquor to } \\
\text { Indians }\end{array}$ & 2nd & $\begin{array}{l}\text { Murphy, } \\
\text { Lawrence }\end{array}$ & $9 / 10 / 1856$ & $\begin{array}{l}27-02-07-02 \mathrm{~F} . \\
60\end{array}$ \\
\hline $\begin{array}{l}\text { Selling Liquor to } \\
\text { Indians }\end{array}$ & $3 \mathrm{rd}$ & Pulaski & $11 / 1 / 1859$ & $\begin{array}{l}27-02-07-03 \mathrm{~F} . \\
80\end{array}$ \\
\hline $\begin{array}{l}\text { Selling Liquor to } \\
\text { Indians }\end{array}$ & 2nd & Red, D. & $5 / 31 / 1856$ & $\begin{array}{l}27-02-07-04 \mathrm{~F} . \\
06\end{array}$ \\
\hline $\begin{array}{l}\text { Selling Liquor to } \\
\text { Indians }\end{array}$ & $3 \mathrm{rd}$ & Richardson, John & $10 / 24 / 1857$ & $\begin{array}{l}27-02-07-04 \mathrm{~F} . \\
23\end{array}$ \\
\hline $\begin{array}{l}\text { Selling Liquor to } \\
\text { Indians }\end{array}$ & 3rd & $\begin{array}{l}\text { Sheldon, } \\
\text { William }\end{array}$ & $9 / 1 / 1858$ & $\begin{array}{l}27-02-07-05 \mathrm{~F} . \\
40\end{array}$ \\
\hline $\begin{array}{l}\text { Selling Liquor to } \\
\text { Indians }\end{array}$ & 2nd & Storm, John & $4 / 25 / 1856$ & $\begin{array}{l}27-02-07-06 \mathrm{~F} . \\
19\end{array}$ \\
\hline $\begin{array}{l}\text { Selling Liquor to } \\
\text { Indians }\end{array}$ & 2nd & Storm, John & $4 / 5 / 1856$ & $\begin{array}{l}27-02-07-06 \mathrm{~F} . \\
19\end{array}$ \\
\hline $\begin{array}{l}\text { Selling Liquor to } \\
\text { Indians }\end{array}$ & $3 \mathrm{rd}$ & Taylor, Dr. J. & $8 / 21 / 1858$ & $\begin{array}{l}27-02-07-06 \mathrm{~F} . \\
37\end{array}$ \\
\hline $\begin{array}{l}\text { Selling Liquor to } \\
\text { Indians }\end{array}$ & 3rd & Ward, Robert & $7 / 20 / 1857$ & $\begin{array}{l}27-02-08-01 \mathrm{~F} . \\
08\end{array}$ \\
\hline $\begin{array}{l}\text { Selling Liquor to } \\
\text { Indians }\end{array}$ & 3rd & Washburn, N.W. & $11 / 1 / 1859$ & $\begin{array}{l}27-02-08-01 \mathrm{~F} . \\
11\end{array}$ \\
\hline $\begin{array}{l}\text { Selling Liquor to } \\
\text { Indians }\end{array}$ & $1 \mathrm{st}$ & Watkins & $6 / 28 / 1859$ & $\begin{array}{l}27-02-08-01 \mathrm{~F} . \\
12\end{array}$ \\
\hline $\begin{array}{l}\text { Selling Liquor to } \\
\text { Indians }\end{array}$ & 3rd & Wells, H.P & $10 / 1 / 1859$ & $\begin{array}{l}27-02-08-01 \mathrm{~F} . \\
28\end{array}$ \\
\hline $\begin{array}{l}\text { Selling Liquor } \\
\text { Without a } \\
\text { License }\end{array}$ & 2nd & $\begin{array}{l}\text { Allen, Asa \& } \\
\text { Gordon, J.C. }\end{array}$ & $6 / 1856$ & $\begin{array}{l}27-02-05-06 \mathrm{~F} . \\
10\end{array}$ \\
\hline $\begin{array}{l}\text { Selling Liquor } \\
\text { Without a } \\
\text { License }\end{array}$ & $1 \mathrm{st}$ & Alous, Richard & $4 / 1858$ & $\begin{array}{l}27-02-05-06 \mathrm{~F} . \\
14\end{array}$ \\
\hline $\begin{array}{l}\text { Selling Liquor } \\
\text { Without a } \\
\text { License }\end{array}$ & 3rd & Ballard, Richard & $3 / 1860$ & $\begin{array}{l}27-02-05-06 \mathrm{~F} . \\
39\end{array}$ \\
\hline $\begin{array}{l}\text { Selling Liquor } \\
\text { Without a } \\
\text { License }\end{array}$ & 2nd & Bassett, William & $4 / 1 / 1856$ & $\begin{array}{l}27-02-05-06 \mathrm{~F} . \\
48\end{array}$ \\
\hline $\begin{array}{l}\text { Selling Liquor } \\
\text { Without a } \\
\text { License }\end{array}$ & 2nd & $\begin{array}{l}\text { Bickerstaff, } \\
\text { Sanford }\end{array}$ & $11 / 1856$ & $\begin{array}{l}27-02-05-07 \mathrm{~F} . \\
03\end{array}$ \\
\hline $\begin{array}{l}\text { Selling Liquor } \\
\text { Without a } \\
\text { License }\end{array}$ & 2nd & $\begin{array}{l}\text { Bickerstaff, } \\
\text { Sanford }\end{array}$ & $9 / 22 / 1856$ & $\begin{array}{l}27-02-05-07 \mathrm{~F} . \\
04\end{array}$ \\
\hline $\begin{array}{l}\text { Selling Liquor } \\
\text { Without a } \\
\text { License }\end{array}$ & $1 \mathrm{st}$ & Biglow, Jonathan & $4 / 1 / 1856$ & $\begin{array}{l}27-02-05-07 \mathrm{~F} . \\
08\end{array}$ \\
\hline
\end{tabular}




\begin{tabular}{|c|c|c|c|c|}
\hline $\begin{array}{l}\text { Selling Liquor } \\
\text { Without a } \\
\text { License }\end{array}$ & 2nd & Boles, J.G. & $5 / 10 / 1858$ & $\begin{array}{l}27-02-05-07 \mathrm{~F} \\
16\end{array}$ \\
\hline $\begin{array}{l}\text { Selling Liquor } \\
\text { Without a } \\
\text { License }\end{array}$ & $3 \mathrm{rd}$ & Carey, William & $10 / 29 / 1857$ & $\begin{array}{l}27-02-06-01 \mathrm{~F} \\
03\end{array}$ \\
\hline $\begin{array}{l}\text { Selling Liquor } \\
\text { Without a } \\
\text { License }\end{array}$ & 2nd & $\begin{array}{l}\text { Carmichael, } \\
\text { Henry }\end{array}$ & $6 / 1856$ & $\begin{array}{l}27-02-06-01 \mathrm{~F} \\
06\end{array}$ \\
\hline $\begin{array}{l}\text { Selling Liquor } \\
\text { Without a } \\
\text { License }\end{array}$ & 2nd & Collins, Daniel & $5 / 4 / 1857$ & $\begin{array}{l}27-02-06-02 \mathrm{~F} \\
10\end{array}$ \\
\hline $\begin{array}{l}\text { Selling Liquor } \\
\text { Without a } \\
\text { License }\end{array}$ & $1 \mathrm{st}$ & $\begin{array}{l}\text { Conway, } \\
\text { Michael }\end{array}$ & $4 / 28 / 1858$ & $\begin{array}{l}27-02-06-02 \mathrm{~F} \\
16\end{array}$ \\
\hline $\begin{array}{l}\text { Selling Liquor } \\
\text { Without a } \\
\text { License }\end{array}$ & $1 \mathrm{st}$ & Doyle, Patrick & $4 / 1858$ & $\begin{array}{l}27-02-06-03 \mathrm{~F} \\
47\end{array}$ \\
\hline $\begin{array}{l}\text { Selling Liquor } \\
\text { Without a } \\
\text { License }\end{array}$ & $1 \mathrm{st}$ & Fahey, Tomas & $4 / 30 / 1858$ & $\begin{array}{l}27-02-06-04 \mathrm{~F} \\
17\end{array}$ \\
\hline $\begin{array}{l}\text { Selling Liquor } \\
\text { Without a } \\
\text { License }\end{array}$ & 2nd & $\begin{array}{l}\text { Fishmaker, } \\
\text { Charles }\end{array}$ & $4 / 1 / 1856$ & $\begin{array}{l}27-02-06-04 \mathrm{~F} \\
35\end{array}$ \\
\hline $\begin{array}{l}\text { Selling Liquor } \\
\text { Without a } \\
\text { License }\end{array}$ & 2nd & Fleshman, James & $3 / 1 / 1858$ & $\begin{array}{l}27-02-06-04 \mathrm{~F} \\
37\end{array}$ \\
\hline $\begin{array}{l}\text { Selling Liquor } \\
\text { Without a } \\
\text { License }\end{array}$ & $1 \mathrm{st}$ & Frazier, Hugh & $4 / 4 / 1858$ & $\begin{array}{l}27-02-06-04 \mathrm{~F} \\
49\end{array}$ \\
\hline $\begin{array}{l}\text { Selling Liquor } \\
\text { Without a } \\
\text { License }\end{array}$ & 2nd & $\begin{array}{l}\text { Freeman. } \\
\text { Charles }\end{array}$ & $1 / 1 / 1858$ & $\begin{array}{l}27-02-06-04 \mathrm{~F} \\
51\end{array}$ \\
\hline $\begin{array}{l}\text { Selling Liquor } \\
\text { Without a } \\
\text { License }\end{array}$ & 2nd & Fry, Samuel & $3 / 31 / 1858$ & $\begin{array}{l}27-02-06-04 \mathrm{~F} \\
53\end{array}$ \\
\hline $\begin{array}{l}\text { Selling Liquor } \\
\text { Without a } \\
\text { License }\end{array}$ & 1st, 2nd & Fuller, James & $4 / 1 / 1856$ & $\begin{array}{l}27-02-06-04 \mathrm{~F} \\
57\end{array}$ \\
\hline $\begin{array}{l}\text { Selling Liquor } \\
\text { Without a } \\
\text { License }\end{array}$ & 1st, 2nd & Fuller, James & $4 / 1856$ & $\begin{array}{l}27-02-06-04 \mathrm{~F} \\
56\end{array}$ \\
\hline $\begin{array}{l}\text { Selling Liquor } \\
\text { Without a } \\
\text { License }\end{array}$ & 2nd & Gardner, A. & $2 / 24 / 1859$ & $\begin{array}{l}27-02-06-05 \mathrm{~F} \\
07\end{array}$ \\
\hline $\begin{array}{l}\text { Selling Liquor } \\
\text { Without a } \\
\text { License }\end{array}$ & 2nd & Geargart & $5 / 20 / 1856$ & $\begin{array}{l}27-02-06-05 \mathrm{~F} \\
14\end{array}$ \\
\hline $\begin{array}{l}\text { Selling Liquor } \\
\text { Without a } \\
\text { License }\end{array}$ & 2nd & Haug, George & $3 / 1 / 1856$ & $\begin{array}{l}27-02-06-05 \mathrm{~F} \\
49\end{array}$ \\
\hline $\begin{array}{l}\text { Selling Liquor } \\
\text { Without a } \\
\text { License }\end{array}$ & 2nd & Hicks, Robert & $9 / 1 / 1856$ & $\begin{array}{l}27-02-06-06 \mathrm{~F} \\
08\end{array}$ \\
\hline $\begin{array}{l}\text { Selling Liquor } \\
\text { Without a } \\
\text { License }\end{array}$ & 2nd & Hicks, Robert & $9 / 5 / 1856$ & $\begin{array}{l}27-02-06-06 \mathrm{~F} \\
08\end{array}$ \\
\hline
\end{tabular}




\begin{tabular}{|c|c|c|c|c|}
\hline $\begin{array}{l}\text { Selling Liquor } \\
\text { Without a } \\
\text { License }\end{array}$ & $1 \mathrm{st}$ & Hindman, A. & $4 / 1 / 1856$ & $\begin{array}{l}27-02-06-06 \mathrm{~F} \\
11\end{array}$ \\
\hline $\begin{array}{l}\text { Selling Liquor } \\
\text { Without a } \\
\text { License }\end{array}$ & 2nd & Hindman, A.C. & $4 / 1 / 1856$ & $\begin{array}{l}27-02-06-06 \mathrm{~F} \\
11\end{array}$ \\
\hline $\begin{array}{l}\text { Selling Liquor } \\
\text { Without a } \\
\text { License }\end{array}$ & 2nd & John & $6 / 10 / 1856$ & $\begin{array}{l}27-02-06-07 \mathrm{~F} \\
01\end{array}$ \\
\hline $\begin{array}{l}\text { Selling Liquor } \\
\text { Without a } \\
\text { License }\end{array}$ & $1 \mathrm{st}$ & Johnson, Andrew & $6 / 1 / 1856$ & $\begin{array}{l}27-02-06-07 \mathrm{~F} \\
02\end{array}$ \\
\hline $\begin{array}{l}\text { Selling Liquor } \\
\text { Without a } \\
\text { License }\end{array}$ & 2nd & $\begin{array}{l}\text { Johnson, } \\
\text { Benjamin }\end{array}$ & $7 / 3 / 1857$ & $\begin{array}{l}27-02-06-07 \mathrm{~F} \\
04\end{array}$ \\
\hline $\begin{array}{l}\text { Selling Liquor } \\
\text { Without a } \\
\text { License }\end{array}$ & 2nd & Johnson, Jacob & $6 / 1 / 1856$ & $\begin{array}{l}27-02-06-07 \mathrm{~F} \\
06\end{array}$ \\
\hline $\begin{array}{l}\text { Selling Liquor } \\
\text { Without a } \\
\text { License }\end{array}$ & 2nd & Kelley, Thomas & $5 / 22 / 1856$ & $\begin{array}{l}27-02-06-07 \mathrm{~F} \\
33\end{array}$ \\
\hline $\begin{array}{l}\text { Selling Liquor } \\
\text { Without a } \\
\text { License }\end{array}$ & $1 \mathrm{st}$ & Kerwin, Thomas & $4 / 29 / 1858$ & $\begin{array}{l}27-02-06-07 \mathrm{~F} \\
42\end{array}$ \\
\hline $\begin{array}{l}\text { Selling Liquor } \\
\text { Without a } \\
\text { License }\end{array}$ & 2nd & $\begin{array}{l}\text { Luddington, } \\
\text { Reuben }\end{array}$ & $1 / 30 / 1858$ & $\begin{array}{l}27-02-07-01 \mathrm{~F} \\
42\end{array}$ \\
\hline $\begin{array}{l}\text { Selling Liquor } \\
\text { Without a } \\
\text { License }\end{array}$ & $1 \mathrm{st}$ & McDaniel, John & $4 / 29 / 1860$ & $\begin{array}{l}27-02-07-01 \mathrm{~F} \\
67\end{array}$ \\
\hline $\begin{array}{l}\text { Selling Liquor } \\
\text { Without a } \\
\text { License }\end{array}$ & 1st, 2nd & $\begin{array}{l}\text { McKinney, } \\
\text { William }\end{array}$ & $4 / 1 / 1856$ & $\begin{array}{l}27-02-07-01 \mathrm{~F} \\
81\end{array}$ \\
\hline $\begin{array}{l}\text { Selling Liquor } \\
\text { Without a } \\
\text { License }\end{array}$ & $1 \mathrm{st}$ & Milne, David & $2 / 1 / 1856$ & $\begin{array}{l}27-02-07-02 \mathrm{~F} \\
18\end{array}$ \\
\hline $\begin{array}{l}\text { Selling Liquor } \\
\text { Without a } \\
\text { License }\end{array}$ & $1 \mathrm{st}$ & Milne, David & $4 / 1856$ & $\begin{array}{l}27-02-07-02 \mathrm{~F} \\
18\end{array}$ \\
\hline $\begin{array}{l}\text { Selling Liquor } \\
\text { Without a } \\
\text { License }\end{array}$ & $1 \mathrm{st}$ & $\begin{array}{l}\text { Morgues, } \\
\text { Augustus }\end{array}$ & $2 / 7 / 1857$ & $\begin{array}{l}27-02-07-02 \mathrm{~F} \\
44\end{array}$ \\
\hline $\begin{array}{l}\text { Selling Liquor } \\
\text { Without a } \\
\text { License }\end{array}$ & $1 \mathrm{st}$ & $\begin{array}{l}\text { Murphy, } \\
\text { Lawrence }\end{array}$ & $12 / 1 / 1855$ & $\begin{array}{l}27-02-07-02 \mathrm{~F} \\
55\end{array}$ \\
\hline $\begin{array}{l}\text { Selling Liquor } \\
\text { Without a } \\
\text { License }\end{array}$ & $1 \mathrm{st}$ & $\begin{array}{l}\text { Murphy, } \\
\text { Lawrence }\end{array}$ & $4 / 1 / 1856$ & $\begin{array}{l}27-02-07-02 \mathrm{~F} \\
55\end{array}$ \\
\hline $\begin{array}{l}\text { Selling Liquor } \\
\text { Without a } \\
\text { License }\end{array}$ & $1 \mathrm{st}$ & $\begin{array}{l}\text { Murphy, } \\
\text { Lawrence }\end{array}$ & $8 / 28 / 1856$ & $\begin{array}{l}27-02-07-02 \mathrm{~F} \\
55\end{array}$ \\
\hline $\begin{array}{l}\text { Selling Liquor } \\
\text { Without a } \\
\text { License }\end{array}$ & $1 \mathrm{st}$ & Partin, Martin & $9 / 1 / 1857$ & $\begin{array}{l}27-02-07-03 \mathrm{~F} \\
30\end{array}$ \\
\hline $\begin{array}{l}\text { Selling Liquor } \\
\text { Without a } \\
\text { License }\end{array}$ & $1 \mathrm{st}$ & $\begin{array}{l}\text { Rhoefus, } \\
\text { Fredrick }\end{array}$ & $4 / 11 / 1857$ & $\begin{array}{l}27-02-07-04 \mathrm{~F} \\
13\end{array}$ \\
\hline
\end{tabular}




\begin{tabular}{|c|c|c|c|c|}
\hline $\begin{array}{l}\text { Selling Liquor } \\
\text { Without a } \\
\text { License }\end{array}$ & $1 \mathrm{st}$ & Ringold, George & $4 / 30 / 1858$ & $\begin{array}{l}\text { 27-02-07-04 F. } \\
30\end{array}$ \\
\hline $\begin{array}{l}\text { Selling Liquor } \\
\text { Without a } \\
\text { License }\end{array}$ & $1 \mathrm{st}$ & Rucker, James & $9 / 2 / 1858$ & $\begin{array}{l}27-02-07-04 \mathrm{~F} . \\
46\end{array}$ \\
\hline $\begin{array}{l}\text { Selling Liquor } \\
\text { Without a } \\
\text { License }\end{array}$ & $1 \mathrm{st}$ & Smith, Merril & $1 / 31 / 1858$ & $\begin{array}{l}27-02-07-05 \mathrm{~F} . \\
54\end{array}$ \\
\hline $\begin{array}{l}\text { Selling Liquor } \\
\text { Without a } \\
\text { License }\end{array}$ & 2nd & $\begin{array}{l}\text { Sotterwhite, } \\
\text { Walter }\end{array}$ & $6 / 13 / 1856$ & $\begin{array}{l}27-02-07-05 \mathrm{~F} . \\
11\end{array}$ \\
\hline $\begin{array}{l}\text { Selling Liquor } \\
\text { Without a } \\
\text { License }\end{array}$ & 2nd & $\begin{array}{l}\text { Spradling, } \\
\text { Obediah }\end{array}$ & $1 / 4 / 1858$ & $\begin{array}{l}27-02-07-05 \mathrm{~F} . \\
72\end{array}$ \\
\hline $\begin{array}{l}\text { Selling Liquor } \\
\text { Without a } \\
\text { License }\end{array}$ & 2nd & Storm, John & $2 / 19 / 1856$ & $\begin{array}{l}27-02-07-06 \mathrm{~F} . \\
20\end{array}$ \\
\hline $\begin{array}{l}\text { Selling Liquor } \\
\text { Without a } \\
\text { License }\end{array}$ & 2nd & Storm, John & $5 / 10 / 1856$ & $\begin{array}{l}27-02-07-06 \mathrm{~F} . \\
20\end{array}$ \\
\hline $\begin{array}{l}\text { Selling Liquor } \\
\text { Without a } \\
\text { License }\end{array}$ & 2nd & $\begin{array}{l}\text { Sylverster, } \\
\text { Emery }\end{array}$ & $1 / 1 / 1858$ & $\begin{array}{l}27-02-07-06 \mathrm{~F} . \\
33\end{array}$ \\
\hline $\begin{array}{l}\text { Selling Liquor } \\
\text { Without a } \\
\text { License }\end{array}$ & $1 \mathrm{st}$ & Tzattskowski & $4 / 29 / 1858$ & $\begin{array}{l}27-02-07-07 \mathrm{~F} . \\
15\end{array}$ \\
\hline $\begin{array}{l}\text { Selling Liquor } \\
\text { Without a } \\
\text { License }\end{array}$ & 2nd & $\begin{array}{l}\text { Wallace, } \\
\text { Milliagan }\end{array}$ & $4 / 1 / 1856$ & $\begin{array}{l}27-02-08-01 \mathrm{~F} . \\
03\end{array}$ \\
\hline $\begin{array}{l}\text { Selling Liquor } \\
\text { Without a } \\
\text { License }\end{array}$ & 2nd & $\begin{array}{l}\text { Wallace, } \\
\text { Milligan }\end{array}$ & $4 / 2 / 1856$ & $\begin{array}{l}\text { 27-02-08-01 F. } \\
03\end{array}$ \\
\hline $\begin{array}{l}\text { Selling Liquor } \\
\text { Without a } \\
\text { License }\end{array}$ & 2nd & Waysman, J.K. & $8 / 10 / 1856$ & $\begin{array}{l}27-02-08-01 \mathrm{~F} . \\
17\end{array}$ \\
\hline $\begin{array}{l}\text { Selling Liquor } \\
\text { Without a } \\
\text { License }\end{array}$ & 2nd & Waysman, J.K. & $9 / 10 / 1856$ & $\begin{array}{l}27-02-08-01 \mathrm{~F} . \\
17\end{array}$ \\
\hline $\begin{array}{l}\text { Selling Liquor } \\
\text { Without a } \\
\text { License }\end{array}$ & 2nd & Webb, Garland & $4 / 6 / 1857$ & $\begin{array}{l}27-02-08-01 \mathrm{~F} . \\
23\end{array}$ \\
\hline $\begin{array}{l}\text { Selling Liquor } \\
\text { Without a } \\
\text { License }\end{array}$ & 2nd & $\begin{array}{l}\text { William \& } \\
\text { Webb, Garland }\end{array}$ & $4 / 6 / 1857$ & $\begin{array}{l}27-02-08-01 \mathrm{~F} . \\
47\end{array}$ \\
\hline $\begin{array}{l}\text { Selling Liquor } \\
\text { Without a } \\
\text { License }\end{array}$ & 2nd & $\begin{array}{l}\text { Williamson, } \\
\text { William }\end{array}$ & $2 / 24 / 1859$ & $\begin{array}{l}27-02-08-01 \mathrm{~F} . \\
59\end{array}$ \\
\hline $\begin{array}{l}\text { Selling Liquor } \\
\text { Without a } \\
\text { License }\end{array}$ & 2nd & $\begin{array}{l}\text { Williamson, } \\
\text { William }\end{array}$ & $3 / 1 / 1858$ & $\begin{array}{l}27-02-08-01 \mathrm{~F} . \\
58\end{array}$ \\
\hline $\begin{array}{l}\text { Selling Liquor } \\
\text { Without a } \\
\text { License }\end{array}$ & $3 \mathrm{rd}$ & Wilson, E. & $10 / 30 / 1857$ & $\begin{array}{l}27-02-08-01 \mathrm{~F} . \\
63\end{array}$ \\
\hline $\begin{array}{l}\text { Selling Liquor } \\
\text { Without a } \\
\text { License }\end{array}$ & $1 \mathrm{st}$ & $\begin{array}{l}\text { Yocum, Frncis \& } \\
\text { Fuller, Daniel }\end{array}$ & $2 / 7 / 1857$ & $\begin{array}{l}27-02-08-01 \mathrm{~F} . \\
88\end{array}$ \\
\hline
\end{tabular}




\begin{tabular}{|c|c|c|c|c|}
\hline $\begin{array}{l}\text { Selling Liquor } \\
\text { Without a } \\
\text { License }\end{array}$ & 2nd & Hicks, Robert & $9 / 10 / 1856$ & $\begin{array}{l}27-02-06-06 \mathrm{~F} . \\
05\end{array}$ \\
\hline $\begin{array}{l}\text { Selling } \\
\text { Merchandise to } \\
\text { Indians }\end{array}$ & $3 \mathrm{rd}$ & Ward, Robert & $8 / 1 / 1857$ & $\begin{array}{l}\text { 27-02-08-01 F. } \\
09\end{array}$ \\
\hline $\begin{array}{l}\text { Selling Whiskey } \\
\text { to Indians }\end{array}$ & 3rd & Jones, Eli & $3 / 1 / 1859$ & $\begin{array}{l}27-02-06-07 \mathrm{~F} . \\
12\end{array}$ \\
\hline $\begin{array}{l}\text { Selling Whiskey } \\
\text { Without a license }\end{array}$ & 2 nd & Sellers & $5 / 10 / 1858$ & $\begin{array}{l}27-02-07-05 \mathrm{~F} . \\
29\end{array}$ \\
\hline $\begin{array}{l}\text { Settling on } \\
\text { Indian Land }\end{array}$ & Linn Cnty. & Donahue, Jesse & $10 / 12 / 1860$ & $\begin{array}{l}27-02-06-03 \mathrm{~F} . \\
34\end{array}$ \\
\hline $\begin{array}{l}\text { Settling on } \\
\text { Indian Land }\end{array}$ & Lykins Cnty. & Donoho, Jesse & $12 / 3 / 1859$ & $\begin{array}{l}27-02-06-03 \mathrm{~F} . \\
33\end{array}$ \\
\hline $\begin{array}{l}\text { Settling on } \\
\text { Indian Land }\end{array}$ & 3rd & Green, John & $2 / 1 / 1859$ & $\begin{array}{l}27-02-06-05 \mathrm{~F} . \\
38\end{array}$ \\
\hline $\begin{array}{l}\text { Settling on } \\
\text { Indian Land }\end{array}$ & $3 \mathrm{rd}$ & Griffin, Carter & $12 / 3 / 1859$ & $\begin{array}{l}27-02-06-05 \mathrm{~F} . \\
39\end{array}$ \\
\hline $\begin{array}{l}\text { Settling on } \\
\text { Indian Land }\end{array}$ & 3rd & Kennedy, James & $4 / 19 / 1860$ & $\begin{array}{l}27-02-06-07 \mathrm{~F} . \\
39\end{array}$ \\
\hline $\begin{array}{l}\text { Settling on } \\
\text { Indian Land }\end{array}$ & 3rd & $\begin{array}{l}\text { Lamberth, } \\
\text { Crockett }\end{array}$ & $2 / 1 / 1860$ & $\begin{array}{l}27-02-07-01 \mathrm{~F} . \\
04\end{array}$ \\
\hline $\begin{array}{l}\text { Settling on } \\
\text { Indian Land }\end{array}$ & $3 \mathrm{rd}$ & $\begin{array}{l}\text { Lamberth, } \\
\text { Richard }\end{array}$ & $11 / 1 / 1859$ & $\begin{array}{l}27-02-07-01 \mathrm{~F} . \\
05\end{array}$ \\
\hline $\begin{array}{l}\text { Settling on } \\
\text { Indian Land }\end{array}$ & 3 rd & Littlepage, John & $12 / 3 / 1859$ & $\begin{array}{l}27-02-07-01 \mathrm{~F} . \\
24\end{array}$ \\
\hline $\begin{array}{l}\text { Settling on } \\
\text { Indian Land }\end{array}$ & 2nd & Miller, J. & $10 / 9 / 1858$ & $\begin{array}{l}27-02-07-02 \mathrm{~F} . \\
09\end{array}$ \\
\hline $\begin{array}{l}\text { Settling on } \\
\text { Indian Land }\end{array}$ & 2nd & Miller, Richard & $7 / 16 / 1859$ & $\begin{array}{l}27-02-07-02 \mathrm{~F} . \\
12\end{array}$ \\
\hline $\begin{array}{l}\text { Settling on } \\
\text { Indian Land }\end{array}$ & 3 rd & Patrick, Allen & $12 / 1 / 1859$ & $\begin{array}{l}27-02-07-03 \mathrm{~F} . \\
39\end{array}$ \\
\hline $\begin{array}{l}\text { Settling on } \\
\text { Indian Land }\end{array}$ & 3rd & Phelps, William & $12 / 1 / 1859$ & $\begin{array}{l}27-02-07-03 \mathrm{~F} . \\
50\end{array}$ \\
\hline $\begin{array}{l}\text { Settling on } \\
\text { Indian Land }\end{array}$ & 3rd & Prior, John & $1 / 1 / 1858$ & $\begin{array}{l}27-02-07-03 \mathrm{~F} . \\
79\end{array}$ \\
\hline $\begin{array}{l}\text { Settling on } \\
\text { Indian Land }\end{array}$ & 3rd & Rockwell & $4 / 19 / 1860$ & $\begin{array}{l}27-02-07-04 \mathrm{~F} . \\
39\end{array}$ \\
\hline $\begin{array}{l}\text { Settling on } \\
\text { Indian Land }\end{array}$ & $3 \mathrm{rd}$ & Sage, Charles & $11 / 1 / 1859$ & $\begin{array}{l}27-02-07-05 \mathrm{~F} . \\
06\end{array}$ \\
\hline $\begin{array}{l}\text { Settling on } \\
\text { Indian Land }\end{array}$ & 2nd & Sage, Henry & $3 / 6 / 1860$ & $\begin{array}{l}27-02-07-05 \mathrm{~F} . \\
03\end{array}$ \\
\hline $\begin{array}{l}\text { Settling on } \\
\text { Indian Land }\end{array}$ & 3 rd & Schrivor, John & $12 / 3 / 1859$ & $\begin{array}{l}27-02-07-05 \mathrm{~F} . \\
16\end{array}$ \\
\hline $\begin{array}{l}\text { Settling on } \\
\text { Indian Land }\end{array}$ & $3 \mathrm{rd}$ & Shriver, John & $12 / 1 / 1859$ & $\begin{array}{l}27-02-07-05 \mathrm{~F} . \\
45\end{array}$ \\
\hline $\begin{array}{l}\text { Settling on } \\
\text { Indian Land }\end{array}$ & $3 \mathrm{rd}$ & Shull, Alfred & $4 / 19 / 1860$ & $\begin{array}{l}27-02-07-05 \mathrm{~F} . \\
47\end{array}$ \\
\hline $\begin{array}{l}\text { Settling on } \\
\text { Indian Land }\end{array}$ & Lykins Cnty. & Shull, Alfred & $12 / 1 / 1859$ & $\begin{array}{l}27-02-07-05 \mathrm{~F} . \\
47\end{array}$ \\
\hline $\begin{array}{l}\text { Settling on } \\
\text { Indian Land }\end{array}$ & $3 \mathrm{rd}$ & Smith, Robert & $1 / 1 / 1858$ & $\begin{array}{l}27-02-07-05 \mathrm{~F} . \\
56\end{array}$ \\
\hline $\begin{array}{l}\text { Settling on } \\
\text { Indian Land }\end{array}$ & $3 \mathrm{rd}$ & Taylor, Martin & $1 / 1 / 1860$ & $\begin{array}{l}27-02-07-06 \mathrm{~F} . \\
41\end{array}$ \\
\hline $\begin{array}{l}\text { Settling on } \\
\text { Indian Land }\end{array}$ & $3 \mathrm{rd}$ & Williams, N.R. & $11 / 1 / 1859$ & $\begin{array}{l}27-02-08-01 \mathrm{~F} . \\
55\end{array}$ \\
\hline
\end{tabular}




\begin{tabular}{|c|c|c|c|c|}
\hline $\begin{array}{l}\text { Settling on } \\
\text { Indian Land }\end{array}$ & 3rd & Wilson, Jeremiah & $12 / 3 / 1859$ & $\begin{array}{l}27-02-08-01 \mathrm{~F} . \\
66\end{array}$ \\
\hline $\begin{array}{l}\text { Settling on } \\
\text { Indian Land }\end{array}$ & $3 \mathrm{rd}$ & Feely, Norris & $11 / 1 / 1859$ & $\begin{array}{l}27-02-06-04 \mathrm{~F} . \\
27\end{array}$ \\
\hline $\begin{array}{l}\text { Settling on } \\
\text { Indian Lands }\end{array}$ & $3 \mathrm{rd}$ & $\begin{array}{l}\text { Baldwin, } \\
\text { William }\end{array}$ & $10 / 12 / 1860$ & $\begin{array}{l}27-02-05-06 \mathrm{~F} . \\
31\end{array}$ \\
\hline $\begin{array}{l}\text { Settling on } \\
\text { Indian Lands }\end{array}$ & 3rd & Ball, Parker & $12 / 3 / 1859$ & $\begin{array}{l}27-02-05-06 \mathrm{~F} . \\
32\end{array}$ \\
\hline $\begin{array}{l}\text { Settling on } \\
\text { Indian Lands }\end{array}$ & $3 \mathrm{rd}$ & Ball, Samuel & $12 / 3 / 1859$ & $\begin{array}{l}27-02-05-06 \mathrm{~F} . \\
33\end{array}$ \\
\hline $\begin{array}{l}\text { Settling on } \\
\text { Indian Lands }\end{array}$ & $3 \mathrm{rd}$ & Ballard, Nancy & $10 / 13 / 1860$ & $\begin{array}{l}27-02-05-06 \mathrm{~F} . \\
36\end{array}$ \\
\hline $\begin{array}{l}\text { Settling on } \\
\text { Indian Lands }\end{array}$ & 3rd & Ballard,Richard & $11 / 20 / 1859$ & $\begin{array}{l}27-02-05-06 \mathrm{~F} . \\
37\end{array}$ \\
\hline $\begin{array}{l}\text { Settling on } \\
\text { Indian Lands }\end{array}$ & $3 \mathrm{rd}$ & Bell, John & $1 / 15 / 1860$ & $\begin{array}{l}27-02-05-06 \mathrm{~F} . \\
31\end{array}$ \\
\hline $\begin{array}{l}\text { Settling on } \\
\text { Indian Lands }\end{array}$ & 3rd & Brookins, John & $11 / 1859$ & $\begin{array}{l}27-02-05-07 \mathrm{~F} . \\
37\end{array}$ \\
\hline $\begin{array}{l}\text { Settling on } \\
\text { Indian Lands }\end{array}$ & $3 \mathrm{rd}$ & Cox, James & $1 / 1 / 1859$ & $\begin{array}{l}27-02-06-02 \mathrm{~F} . \\
24\end{array}$ \\
\hline $\begin{array}{l}\text { Settling on } \\
\text { Indian Lands }\end{array}$ & $3 \mathrm{rd}$ & Delf, Andew & $1 / 8 / 1856$ & $\begin{array}{l}27-02-06-03 \mathrm{~F} . \\
23\end{array}$ \\
\hline $\begin{array}{l}\text { Settling on } \\
\text { Indian Lands }\end{array}$ & 3rd & Devillers, Henry & $11 / 1 / 1859$ & $\begin{array}{l}27-02-06-03 \mathrm{~F} . \\
24\end{array}$ \\
\hline $\begin{array}{l}\text { Settling on } \\
\text { Indian Lands }\end{array}$ & $3 \mathrm{rd}$ & Griffin, Stephen & $12 / 3 / 1859$ & $\begin{array}{l}27-02-06-05 \mathrm{~F} . \\
40\end{array}$ \\
\hline $\begin{array}{l}\text { Settling on } \\
\text { Indian Lands }\end{array}$ & 3rd & Hayes, Stephen & $12 / 1 / 1859$ & $\begin{array}{l}27-02-06-05 \mathrm{~F} . \\
72\end{array}$ \\
\hline $\begin{array}{l}\text { Settling on } \\
\text { Indian Lands }\end{array}$ & 3rd & $\begin{array}{l}\text { Huttmann, } \\
\text { William }\end{array}$ & $4 / 19 / 1860$ & $\begin{array}{l}27-02-06-06 \mathrm{~F} . \\
29\end{array}$ \\
\hline $\begin{array}{l}\text { Settling on } \\
\text { Indian Lands }\end{array}$ & $3 \mathrm{rd}$ & King, John & $12 / 1 / 1859$ & $\begin{array}{l}27-02-06-07 \mathrm{~F} . \\
54\end{array}$ \\
\hline $\begin{array}{l}\text { Settling on } \\
\text { Indian lands }\end{array}$ & 3rd & Long, David & $1 / 1 / 1858$ & $\begin{array}{l}27-02-07-01 \mathrm{~F} . \\
31\end{array}$ \\
\hline $\begin{array}{l}\text { Settling on } \\
\text { Indian Lands }\end{array}$ & $3 \mathrm{rd}$ & Long, Lewis & $12 / 14 / 1859$ & $\begin{array}{l}27-02-07-01 \mathrm{~F} . \\
33\end{array}$ \\
\hline $\begin{array}{l}\text { Settling on } \\
\text { Indian Lands }\end{array}$ & $1 \mathrm{st}$ & Long, Madison & $1 / 1 / 1858$ & $\begin{array}{l}27-02-07-01 \mathrm{~F} . \\
34\end{array}$ \\
\hline $\begin{array}{l}\text { Settling on } \\
\text { Indian Lands }\end{array}$ & 3rd & Long, Samuel & $12 / 3 / 1859$ & $\begin{array}{l}27-02-07-01 \mathrm{~F} . \\
36\end{array}$ \\
\hline $\begin{array}{l}\text { Settling on } \\
\text { Indian Lands }\end{array}$ & $3 \mathrm{rd}$ & $\begin{array}{l}\text { McDonald, } \\
\text { Abraham }\end{array}$ & $4 / 19 / 1860$ & $\begin{array}{l}27-02-07-01 \mathrm{~F} . \\
69\end{array}$ \\
\hline $\begin{array}{l}\text { Settling on } \\
\text { Indian Lands }\end{array}$ & 3rd & $\begin{array}{l}\text { McDonald, } \\
\text { Henry }\end{array}$ & $1 / 1 / 1858$ & $\begin{array}{l}27-02-07-01 \mathrm{~F} . \\
70\end{array}$ \\
\hline $\begin{array}{l}\text { Settling on } \\
\text { Indian Lands }\end{array}$ & 3 rd & $\begin{array}{l}\text { McMahon, } \\
\text { David }\end{array}$ & $10 / 12 / 1860$ & $\begin{array}{l}27-02-07-01 \mathrm{~F} . \\
86\end{array}$ \\
\hline $\begin{array}{l}\text { Settling on } \\
\text { Indian Lands }\end{array}$ & 3rd & Moore, George & $11 / 1 / 1859$ & $\begin{array}{l}27-02-07-02 \mathrm{~F} . \\
38\end{array}$ \\
\hline $\begin{array}{l}\text { Settling on } \\
\text { Indian Lands }\end{array}$ & 3 rd & Palmer, Asa & $4 / 19 / 1860$ & $\begin{array}{l}27-02-07-03 \mathrm{~F} . \\
23\end{array}$ \\
\hline $\begin{array}{l}\text { Settling on } \\
\text { Indian Lands }\end{array}$ & 3rd & Reed, Anson & $4 / 1 / 1859$ & $\begin{array}{l}27-02-07-04 \mathrm{~F} . \\
09\end{array}$ \\
\hline $\begin{array}{l}\text { Settling on } \\
\text { Indian Lands }\end{array}$ & 3rd & Robert, James & $11 / 1 / 1859$ & $\begin{array}{l}27-02-07-04 \mathrm{~F} . \\
34\end{array}$ \\
\hline $\begin{array}{l}\text { Settling on } \\
\text { Indian Lands }\end{array}$ & 2nd & Roberts, Thomas & $3 / 1 / 1860$ & $\begin{array}{l}27-02-07-04 \mathrm{~F} . \\
35\end{array}$ \\
\hline
\end{tabular}




\begin{tabular}{|c|c|c|c|c|}
\hline $\begin{array}{l}\text { Settling on } \\
\text { Indian Lands }\end{array}$ & 2nd & Sage, James & $7 / 27 / 1859$ & $\begin{array}{l}\text { 27-02-07-05 F. } \\
05\end{array}$ \\
\hline $\begin{array}{l}\text { Settling on } \\
\text { Indian Lands }\end{array}$ & $3 \mathrm{rd}$ & Scott, Julian & $6 / 30 / 1859$ & $\begin{array}{l}27-02-07-05 \mathrm{~F} . \\
20\end{array}$ \\
\hline $\begin{array}{l}\text { Settling on } \\
\text { Indian Lands }\end{array}$ & $3 \mathrm{rd}$ & $\begin{array}{l}\text { Snodgrass, } \\
\text { Charles }\end{array}$ & $1 / 1 / 1858$ & $\begin{array}{l}27-02-07-05 \mathrm{~F} . \\
59\end{array}$ \\
\hline $\begin{array}{l}\text { Settling on } \\
\text { Indian Lands }\end{array}$ & $3 \mathrm{rd}$ & Spangler, Henry & $3 / 1 / 1860$ & $\begin{array}{l}27-02-07-05 \mathrm{~F} . \\
64\end{array}$ \\
\hline $\begin{array}{l}\text { Settling on } \\
\text { Indian Lands }\end{array}$ & 3rd & Weaver, Fanny & $10 / 12 / 1860$ & $\begin{array}{l}27-02-08-01 \mathrm{~F} . \\
19\end{array}$ \\
\hline $\begin{array}{l}\text { Settling on } \\
\text { Indian Lands }\end{array}$ & 3rd & Weaver, Fanny & $10 / 12 / 1860$ & $\begin{array}{l}27-02-08-01 \mathrm{~F} . \\
20\end{array}$ \\
\hline $\begin{array}{l}\text { Settling on } \\
\text { Indian Lands }\end{array}$ & 3rd & Weaver, William & $10 / 12 / 1860$ & $\begin{array}{l}27-02-08-01 \mathrm{~F} . \\
22\end{array}$ \\
\hline $\begin{array}{l}\text { Settling on } \\
\text { Indian Lands }\end{array}$ & $3 \mathrm{rd}$ & Weid, Robert & $1 / 10 / 1856$ & $\begin{array}{l}27-02-07-07 \mathrm{~F} . \\
31\end{array}$ \\
\hline $\begin{array}{l}\text { Settling on } \\
\text { Miami Indian } \\
\text { Land }\end{array}$ & 2nd & Gray, Joseph & $11 / 19 / 1859$ & $\begin{array}{l}27-02-06-05 \mathrm{~F} . \\
36\end{array}$ \\
\hline Shooting & $?$ & McCrea, Cole & $4 / 16 / 1859$ & $\begin{array}{l}27-02-07-01 \mathrm{~F} . \\
62\end{array}$ \\
\hline Stealing & $1 \mathrm{st}$ & Carson. Franklin & $9 / 13 / 1860$ & $\begin{array}{l}27-02-06-01 \mathrm{~F} . \\
12\end{array}$ \\
\hline Stealing & 2nd & Evans, David & $10 / 8 / 1857$ & $\begin{array}{l}27-02-06-04 \mathrm{~F} . \\
12\end{array}$ \\
\hline Stealing & 3rd & Pillips, Henry & $2 / 1 / 1860$ & $\begin{array}{l}27-02-07-03 \mathrm{~F} . \\
57\end{array}$ \\
\hline Stealing & $3 \mathrm{rd}$ & Wilson, John & $5 / 1 / 1857$ & $\begin{array}{l}27-02-08-01 \mathrm{~F} . \\
69\end{array}$ \\
\hline Stealing Cattle & 3rd & Kean & $8 / 1 / 1859$ & $\begin{array}{l}27-02-06-07 \mathrm{~F} . \\
28\end{array}$ \\
\hline Stealing Cattle & $1 \mathrm{st}$ & $\begin{array}{l}\text { Laws, Albert \& } \\
\text { Sours, W.B. }\end{array}$ & $9 / 29 / 1856$ & $\begin{array}{l}27-02-07-01 \mathrm{~F} . \\
12\end{array}$ \\
\hline Stealing Cattle & $3 \mathrm{rd}$ & Tamborsely & $8 / 1 / 1859$ & $\begin{array}{l}27-02-07-06 \mathrm{~F} . \\
36\end{array}$ \\
\hline Stealing Hay & Douglas Cnty. & $\begin{array}{l}\text { Hunsey, } \\
\text { Jonathan }\end{array}$ & $4 / 2 / 1858$ & $\begin{array}{l}27-02-06-06 \mathrm{~F} . \\
40\end{array}$ \\
\hline Stealing Hogs & $?$ & $\begin{array}{l}\text { Bradburry, } \\
\text { James \& } \\
\text { Bradburry, John, } \\
\text { \& Cocker, } \\
\text { Joseph }\end{array}$ & $4 / 30 / 1855$ & $\begin{array}{l}27-02-05-07 \mathrm{~F} . \\
25\end{array}$ \\
\hline Stealing Horses & $?$ & Brown, John & $5 / 1856$ & $\begin{array}{l}27-02-05-07 \mathrm{~F} . \\
43\end{array}$ \\
\hline Stealing Horses & 3rd & $\begin{array}{l}\text { McCumbter, } \\
\text { E.T. }\end{array}$ & $1 / 1 / 1859$ & $\begin{array}{l}27-02-07-01 \mathrm{~F} . \\
65\end{array}$ \\
\hline $\begin{array}{l}\text { Stealing Indian } \\
\text { Horses }\end{array}$ & $1 \mathrm{st}$ & Hart, Jack & $9 / 5 / 1858$ & $\begin{array}{l}27-02-06-05 \mathrm{~F} . \\
58\end{array}$ \\
\hline $\begin{array}{l}\text { Stealing Indian } \\
\text { Horses }\end{array}$ & 3rd & Marble, Lyman & $1 / 1 / 1860$ & $\begin{array}{l}27-02-07-01 \mathrm{~F} . \\
46\end{array}$ \\
\hline $\begin{array}{l}\text { Stealing Indian } \\
\text { Horses }\end{array}$ & $1 \mathrm{st}$ & Newcomb, John & $7 / 30 / 1858$ & $\begin{array}{l}27-02-07-03 \mathrm{~F} . \\
02\end{array}$ \\
\hline $\begin{array}{l}\text { Stealing Indian } \\
\text { Horses }\end{array}$ & 3rd & $\begin{array}{l}\text { Pennington, } \\
\text { James }\end{array}$ & $1 / 1 / 1859$ & $\begin{array}{l}27-02-07-03 \mathrm{~F} . \\
42\end{array}$ \\
\hline $\begin{array}{l}\text { Stealing Indian } \\
\text { Property }\end{array}$ & 3rd & Cott, Samuel & $2 / 1 / 1859$ & $\begin{array}{l}27-02-07-05 \mathrm{~F} . \\
21\end{array}$ \\
\hline
\end{tabular}




\begin{tabular}{|c|c|c|c|c|}
\hline $\begin{array}{l}\text { Stealing Indian } \\
\text { Property }\end{array}$ & $3 \mathrm{rd}$ & Moody, James & $1 / 1 / 1859$ & $\begin{array}{l}27-02-07-02 \mathrm{~F} . \\
36\end{array}$ \\
\hline $\begin{array}{l}\text { Stealing Indian } \\
\text { Property }\end{array}$ & $3 \mathrm{rd}$ & $\begin{array}{l}\text { Northern, } \\
\text { Russell }\end{array}$ & $2 / 1 / 1859$ & $\begin{array}{l}27-02-07-03 \mathrm{~F} . \\
08\end{array}$ \\
\hline $\begin{array}{l}\text { Stealing Indian } \\
\text { Property }\end{array}$ & $3 \mathrm{rd}$ & Parner, Ailliam & $12 / 29 / 1859$ & $\begin{array}{l}27-02-07-03 \mathrm{~F} . \\
29\end{array}$ \\
\hline $\begin{array}{l}\text { Stealing Indian } \\
\text { Property }\end{array}$ & $3 \mathrm{rd}$ & Scott, Julian & $1 / 1 / 1859$ & $\begin{array}{l}27-02-07-05 \mathrm{~F} . \\
19\end{array}$ \\
\hline $\begin{array}{l}\text { Stealing Indian } \\
\text { Property }\end{array}$ & $3 \mathrm{rd}$ & Wright, William & $12 / 29 / 1859$ & $\begin{array}{l}27-02-08-01 \mathrm{~F} . \\
86\end{array}$ \\
\hline $\begin{array}{l}\text { Stealing Indian } \\
\text { Property }\end{array}$ & $3 \mathrm{rd}$ & Scott, Thomas & $2 / 1 / 1859$ & $\begin{array}{l}27-02-07-05 \mathrm{~F} . \\
22\end{array}$ \\
\hline $\begin{array}{l}\text { Stealing Indian } \\
\text { Timber }\end{array}$ & $3 \mathrm{rd}$ & Perry, Joseph & $5 / 1 / 1857$ & $\begin{array}{l}27-02-07-03 \mathrm{~F} . \\
46\end{array}$ \\
\hline $\begin{array}{l}\text { Stealing Indian } \\
\text { Timber }\end{array}$ & $3 \mathrm{rd}$ & Pettros & $11 / 1 / 1859$ & $\begin{array}{l}27-02-07-03 \mathrm{~F} . \\
48\end{array}$ \\
\hline $\begin{array}{l}\text { Stealing Indian } \\
\text { Timber }\end{array}$ & $3 \mathrm{rd}$ & Peyton, Alfred & $11 / 28 / 1859$ & $\begin{array}{l}27-02-07-03 \mathrm{~F} . \\
49\end{array}$ \\
\hline $\begin{array}{l}\text { Stealing Indian } \\
\text { Timber }\end{array}$ & $3 \mathrm{rd}$ & Peyton, Alfred & $12 / 1 / 1859$ & $\begin{array}{l}27-02-07-03 \mathrm{~F} . \\
49\end{array}$ \\
\hline $\begin{array}{l}\text { Stealing Indian } \\
\text { Timber }\end{array}$ & $3 \mathrm{rd}$ & Tuttle, Cyrus & $1 / 1 / 1859$ & $\begin{array}{l}27-02-07-07 \mathrm{~F} . \\
12\end{array}$ \\
\hline Stealing Oxen & 2 nd & $\begin{array}{l}\text { Haselting, } \\
\text { Dalton }\end{array}$ & $5 / 2 / 1857$ & $\begin{array}{l}27-02-06-05 \mathrm{~F} . \\
66\end{array}$ \\
\hline $\begin{array}{l}\text { Stealing Timber } \\
\text { off Indian Lands }\end{array}$ & $3 \mathrm{rd}$ & Green, John & $4 / 1 / 1857$ & $\begin{array}{l}27-02-06-05 \mathrm{~F} . \\
37\end{array}$ \\
\hline $\begin{array}{l}\text { Stealing Timber } \\
\text { off Indian Lands }\end{array}$ & $3 \mathrm{rd}$ & Randall, J. & $11 / 1 / 1859$ & $\begin{array}{l}27-02-07-04 \mathrm{~F} . \\
02\end{array}$ \\
\hline $\begin{array}{l}\text { Stealing Timber } \\
\text { off Indian Lands }\end{array}$ & $3 r d$ & $\begin{array}{l}\text { Westfall, } \\
\text { Abraham }\end{array}$ & $2 / 1 / 1859$ & $\begin{array}{l}27-02-08-01 \mathrm{~F} . \\
34\end{array}$ \\
\hline $\begin{array}{l}\text { Stealing Timber } \\
\text { off Indian Lands }\end{array}$ & $3 \mathrm{rd}$ & Westfall, Abram & $2 / 1 / 1859$ & $\begin{array}{l}27-02-08-01 \mathrm{~F} . \\
35\end{array}$ \\
\hline $\begin{array}{l}\text { Stealing Timber } \\
\text { off Indian Lands }\end{array}$ & $3 \mathrm{rd}$ & White, B.M. & $10 / 1 / 1859$ & $\begin{array}{l}27-02-08-01 \mathrm{~F} . \\
41\end{array}$ \\
\hline $\begin{array}{l}\text { Stolen Property } \\
\text { on Indian Lands }\end{array}$ & $?$ & Cox, Levi & $9 / 4 / 1858$ & $\begin{array}{l}27-02-06-02 \mathrm{~F} . \\
25\end{array}$ \\
\hline Subpoena & 2 nd & Williams, H.H. & $8 / 17 / 1857$ & $\begin{array}{l}27-02-08-01 \mathrm{~F} . \\
49\end{array}$ \\
\hline $\begin{array}{l}\text { Subverting the } \\
\text { Government } \\
\text { (Treason) }\end{array}$ & 2 nd & Brown, John & $5 / 1 / 1856$ & $\begin{array}{l}27-02-05-07 \mathrm{~F} . \\
50\end{array}$ \\
\hline $\begin{array}{l}\text { Surety To Keep } \\
\text { the Peace }\end{array}$ & Douglas Cnty. & Chapman. E. & $12 / 29 / 1854$ & $\begin{array}{l}27-02-06-01 \mathrm{~F} . \\
16\end{array}$ \\
\hline $\begin{array}{l}\text { Surety To Keep } \\
\text { the Peace }\end{array}$ & 1 st & $\begin{array}{l}\text { Johnson, } \\
\text { Nathaniel }\end{array}$ & $2 / 1 / 1855$ & $\begin{array}{l}27-02-06-07 \mathrm{~F} . \\
07\end{array}$ \\
\hline $\begin{array}{l}\text { Taking Indian } \\
\text { Property }\end{array}$ & $3 \mathrm{rd}$ & $\begin{array}{l}\text { Anderson. } \\
\text { Robert }\end{array}$ & $5 / 1859$ & $\begin{array}{l}27-02-05-06 \mathrm{~F} . \\
15\end{array}$ \\
\hline $\begin{array}{l}\text { Taking Indian } \\
\text { Property }\end{array}$ & $3 \mathrm{rd}$ & Barles, John & $3 / 1 / 1859$ & $\begin{array}{l}27-02-05-06 \mathrm{~F} . \\
43\end{array}$ \\
\hline $\begin{array}{l}\text { Taking Indian } \\
\text { Property }\end{array}$ & $3 \mathrm{rd}$ & Bayles, Abijah & $12 / 15 / 1859$ & $\begin{array}{l}27-02-05-06 \mathrm{~F} . \\
54\end{array}$ \\
\hline $\begin{array}{l}\text { Taking Indian } \\
\text { Property }\end{array}$ & $3 \mathrm{rd}$ & Bayles, Abijah & $2 / 1 / 1859$ & $\begin{array}{l}27-02-05-06 \mathrm{~F} . \\
53\end{array}$ \\
\hline $\begin{array}{l}\text { Taking Indian } \\
\text { Property }\end{array}$ & $3 \mathrm{rd}$ & Beck, Henry & $1 / 1 / 1859$ & $\begin{array}{l}27-02-05-06 \mathrm{~F} . \\
55\end{array}$ \\
\hline
\end{tabular}




\begin{tabular}{|c|c|c|c|c|}
\hline $\begin{array}{l}\text { Taking Indian } \\
\text { Property }\end{array}$ & $3 \mathrm{rd}$ & $\begin{array}{l}\text { Beshear } \\
\text { (Brasher), Beld }\end{array}$ & $1 / 1 / 1859$ & $\begin{array}{l}27-02-05-06 \mathrm{~F} . \\
64\end{array}$ \\
\hline $\begin{array}{l}\text { Taking Indian } \\
\text { Property }\end{array}$ & $3 \mathrm{rd}$ & Bevins, John & $5 / 1 / 1859$ & $\begin{array}{l}27-02-05-07 \mathrm{~F} . \\
01\end{array}$ \\
\hline $\begin{array}{l}\text { Taking Indian } \\
\text { Property }\end{array}$ & $3 \mathrm{rd}$ & Brashear, Belt & $5 / 1860$ & $\begin{array}{l}27-02-05-07 \mathrm{~F} . \\
28\end{array}$ \\
\hline $\begin{array}{l}\text { Taking Indian } \\
\text { Property }\end{array}$ & $3 \mathrm{rd}$ & Brasher, William & $1 / 1860$ & $\begin{array}{l}27-02-05-07 \mathrm{~F} . \\
29\end{array}$ \\
\hline $\begin{array}{l}\text { Taking Indian } \\
\text { Property }\end{array}$ & $3 \mathrm{rd}$ & Bray, Seth & $4 / 19 / 1850$ & $\begin{array}{l}27-02-05-07 \mathrm{~F} . \\
32\end{array}$ \\
\hline $\begin{array}{l}\text { Taking Indian } \\
\text { Property }\end{array}$ & $3 \mathrm{rd}$ & Bray, Seth & $5 / 1859$ & $\begin{array}{l}27-02-05-07 \mathrm{~F} . \\
31\end{array}$ \\
\hline $\begin{array}{l}\text { Taking Indian } \\
\text { Property }\end{array}$ & 1 st & $\begin{array}{l}\text { Buckmaster, } \\
\text { Henry }\end{array}$ & $9 / 1858$ & $\begin{array}{l}27-02-05-07 \mathrm{~F} . \\
54\end{array}$ \\
\hline $\begin{array}{l}\text { Taking Indian } \\
\text { Property }\end{array}$ & $3 \mathrm{rd}$ & Chafin, Joseph & $12 / 1859$ & $\begin{array}{l}27-02-06-01 \mathrm{~F} . \\
14\end{array}$ \\
\hline $\begin{array}{l}\text { Taking Indian } \\
\text { Property }\end{array}$ & $3 \mathrm{rd}$ & Chafin. David & $12 / 2 / 1859$ & $\begin{array}{l}27-02-06-01 \mathrm{~F} . \\
15\end{array}$ \\
\hline $\begin{array}{l}\text { Taking Indian } \\
\text { Property }\end{array}$ & 3rd & Chalfont, David & $4 / 1859$ & $\begin{array}{l}27-02-06-01 \mathrm{~F} . \\
01\end{array}$ \\
\hline $\begin{array}{l}\text { Taking Indian } \\
\text { Property }\end{array}$ & $3 \mathrm{rd}$ & Conant, Joseph & $2 / 1 / 1859$ & $\begin{array}{l}27-02-06-02 \mathrm{~F} . \\
13\end{array}$ \\
\hline $\begin{array}{l}\text { Taking Indian } \\
\text { Property }\end{array}$ & $3 \mathrm{rd}$ & Cress, George & $11 / 1 / 1859$ & $\begin{array}{l}27-02-06-02 \mathrm{~F} . \\
32\end{array}$ \\
\hline $\begin{array}{l}\text { Taking Indian } \\
\text { Property }\end{array}$ & $3 \mathrm{rd}$ & $\begin{array}{l}\text { Cromley, } \\
\text { William }\end{array}$ & $2 / 25 / 1859$ & $\begin{array}{l}27-02-06-02 \mathrm{~F} . \\
36\end{array}$ \\
\hline $\begin{array}{l}\text { Taking Indian } \\
\text { Property }\end{array}$ & $3 \mathrm{rd}$ & Cronly, James & 1857 & $\begin{array}{l}27-02-06-02 \mathrm{~F} . \\
34\end{array}$ \\
\hline $\begin{array}{l}\text { Taking Indian } \\
\text { Property }\end{array}$ & $3 \mathrm{rd}$ & Crunley, James & $1 / 1 / 1860$ & $\begin{array}{l}27-02-06-02 \mathrm{~F} . \\
37\end{array}$ \\
\hline $\begin{array}{l}\text { Taking Indian } \\
\text { Property }\end{array}$ & $3 \mathrm{rd}$ & $\begin{array}{l}\text { Donahue, } \\
\text { William \& } \\
\text { Owens, William }\end{array}$ & $7 / 20 / 1859$ & $\begin{array}{l}27-02-06-03 \mathrm{~F} . \\
30\end{array}$ \\
\hline $\begin{array}{l}\text { Taking Indian } \\
\text { Property }\end{array}$ & $3 \mathrm{rd}$ & Forbes, Reuben & $1 / 1 / 1860$ & $\begin{array}{l}27-02-06-04 \mathrm{~F} . \\
43\end{array}$ \\
\hline $\begin{array}{l}\text { Taking Indian } \\
\text { Property }\end{array}$ & $3 \mathrm{rd}$ & $\begin{array}{l}\text { Heckerdon, } \\
\text { Philip }\end{array}$ & $2 / 1 / 1859$ & $\begin{array}{l}27-02-06-06 \mathrm{~F} . \\
01\end{array}$ \\
\hline $\begin{array}{l}\text { Taking Indian } \\
\text { Property }\end{array}$ & $3 \mathrm{rd}$ & Henley, Edward & $1 / 1 / 1859$ & $\begin{array}{l}27-02-06-06 \mathrm{~F} . \\
03\end{array}$ \\
\hline $\begin{array}{l}\text { Taking Indian } \\
\text { Property }\end{array}$ & $3 \mathrm{rd}$ & $\begin{array}{l}\text { Hyatt, Enoch \& } \\
\text { Bayles, Abijah }\end{array}$ & $?$ & $\begin{array}{l}27-02-06-06 \mathrm{~F} . \\
47\end{array}$ \\
\hline $\begin{array}{l}\text { Taking Indian } \\
\text { Property }\end{array}$ & $3 \mathrm{rd}$ & Jones, James & $1 / 1 / 1859$ & $\begin{array}{l}27-02-06-07 \mathrm{~F} . \\
13\end{array}$ \\
\hline $\begin{array}{l}\text { Taking Indian } \\
\text { Property }\end{array}$ & $3 \mathrm{rd}$ & Jones,. James & $2 / 10 / 1859$ & $\begin{array}{l}27-02-06-02 \mathrm{~F} . \\
35\end{array}$ \\
\hline $\begin{array}{l}\text { Taking Indian } \\
\text { Property }\end{array}$ & $3 \mathrm{rd}$ & Kelley, Samuel & $1 / 1 / 1859$ & $\begin{array}{l}27-02-06-07 \mathrm{~F} . \\
32\end{array}$ \\
\hline $\begin{array}{l}\text { Taking Indian } \\
\text { Property }\end{array}$ & $3 \mathrm{rd}$ & Kelly, George & $7 / 1 / 1859$ & $\begin{array}{l}27-02-06-07 \mathrm{~F} . \\
36\end{array}$ \\
\hline $\begin{array}{l}\text { Taking Indian } \\
\text { Property }\end{array}$ & $3 \mathrm{rd}$ & Kimble, Milton & $2 / 1859$ & $\begin{array}{l}27-02-06-07 \mathrm{~F} . \\
53\end{array}$ \\
\hline $\begin{array}{l}\text { Taking Indian } \\
\text { Property }\end{array}$ & $3 \mathrm{rd}$ & $\begin{array}{l}\text { Knowlton, } \\
\text { Charles }\end{array}$ & $1 / 1 / 1859$ & $\begin{array}{l}27-02-06-07 \mathrm{~F} . \\
60\end{array}$ \\
\hline $\begin{array}{l}\text { Taking Indian } \\
\text { Property }\end{array}$ & $3 \mathrm{rd}$ & $\begin{array}{l}\text { Knowlton, } \\
\text { Charles }\end{array}$ & $11 / 1859$ & $\begin{array}{l}27-02-06-07 \mathrm{~F} . \\
60\end{array}$ \\
\hline
\end{tabular}




\begin{tabular}{|c|c|c|c|c|}
\hline $\begin{array}{l}\text { Taking Indian } \\
\text { Property }\end{array}$ & $3 \mathrm{rd}$ & Prescott, Oliver & $2 / 1 / 1859$ & $\begin{array}{l}27-02-07-03 \mathrm{~F} . \\
75\end{array}$ \\
\hline $\begin{array}{l}\text { Taking Indian } \\
\text { Property }\end{array}$ & $3 \mathrm{rd}$ & Prindle, Nathan & $2 / 1 / 1859$ & $\begin{array}{l}27-02-07-03 \mathrm{~F} . \\
78\end{array}$ \\
\hline $\begin{array}{l}\text { Taking Indian } \\
\text { Property }\end{array}$ & $3 r d$ & Redfield, Lee & $2 / 1 / 1859$ & $\begin{array}{l}27-02-07-04 \mathrm{~F} . \\
07\end{array}$ \\
\hline $\begin{array}{l}\text { Taking Indian } \\
\text { Property }\end{array}$ & $3 \mathrm{rd}$ & $\begin{array}{l}\text { Ridsworth, } \\
\text { Robert }\end{array}$ & $2 / 1 / 1859$ & $\begin{array}{l}27-02-07-04 \mathrm{~F} . \\
28\end{array}$ \\
\hline $\begin{array}{l}\text { Taking Indian } \\
\text { Property }\end{array}$ & $3 \mathrm{rd}$ & Sears, Nathan & $2 / 1 / 1859$ & $\begin{array}{l}27-02-07-05 \mathrm{~F} . \\
27\end{array}$ \\
\hline $\begin{array}{l}\text { Taking Indian } \\
\text { Property }\end{array}$ & $3 \mathrm{rd}$ & Smith, Silas & $5 / 1859$ & $\begin{array}{l}27-02-07-05 \mathrm{~F} . \\
58\end{array}$ \\
\hline $\begin{array}{l}\text { Taking Indian } \\
\text { Property }\end{array}$ & $3 \mathrm{rd}$ & Stevens, William & $12 / 2 / 1859$ & $\begin{array}{l}27-02-07-06 \mathrm{~F} . \\
12\end{array}$ \\
\hline $\begin{array}{l}\text { Taking Indian } \\
\text { Property }\end{array}$ & $3 r d$ & White, B.M & $2 / 1859$ & $\begin{array}{l}27-02-08-01 \mathrm{~F} . \\
42\end{array}$ \\
\hline $\begin{array}{l}\text { Taking Indian } \\
\text { Property }\end{array}$ & $3 \mathrm{rd}$ & Williams, Jesse & $12 / 15 / 1859$ & $\begin{array}{l}27-02-08-01 \mathrm{~F} . \\
52\end{array}$ \\
\hline $\begin{array}{l}\text { Taking Indian } \\
\text { Timber }\end{array}$ & $3 \mathrm{rd}$ & enoyer, William & $2 / 1 / 1859$ & $\begin{array}{l}27-02-07-03 \mathrm{~F} . \\
43\end{array}$ \\
\hline $\begin{array}{l}\text { Taking Indian } \\
\text { Timber }\end{array}$ & $3 \mathrm{rd}$ & Phelps, William & $10 / 1 / 1859$ & $\begin{array}{l}27-02-07-03 \mathrm{~F} . \\
51\end{array}$ \\
\hline $\begin{array}{l}\text { Taking Indian } \\
\text { Timber }\end{array}$ & $3 \mathrm{rd}$ & Pigg, John & $10 / 1 / 1858$ & $\begin{array}{l}27-02-07-03 \mathrm{~F} . \\
60\end{array}$ \\
\hline $\begin{array}{l}\text { Taking Indian } \\
\text { Timber }\end{array}$ & $3 \mathrm{rd}$ & Pigg, John & $11 / 1 / 1859$ & $\begin{array}{l}27-02-07-03 \mathrm{~F} . \\
61\end{array}$ \\
\hline $\begin{array}{l}\text { Taking Indian } \\
\text { Timber }\end{array}$ & $3 \mathrm{rd}$ & Pigg, John & $5 / 1 / 1857$ & $\begin{array}{l}27-02-07-03 \mathrm{~F} . \\
60\end{array}$ \\
\hline $\begin{array}{l}\text { Taking Indian } \\
\text { Timber }\end{array}$ & $3 \mathrm{rd}$ & Pigg, William & $5 / 1 / 1857$ & $\begin{array}{l}27-02-07-03 \mathrm{~F} . \\
62\end{array}$ \\
\hline $\begin{array}{l}\text { Taking Indian } \\
\text { Timber }\end{array}$ & $3 \mathrm{rd}$ & Schell, Alfred & $12 / 2 / 1859$ & $\begin{array}{l}27-02-07-05 \mathrm{~F} . \\
15\end{array}$ \\
\hline $\begin{array}{l}\text { Taking Indian } \\
\text { Timber }\end{array}$ & 3rd & Sears, Charles & $11 / 1 / 1859$ & $\begin{array}{l}27-02-07-05 \mathrm{~F} . \\
24\end{array}$ \\
\hline $\begin{array}{l}\text { Taking Indian } \\
\text { Timber }\end{array}$ & $3 \mathrm{rd}$ & Sears, Nathan & $11 / 1 / 1859$ & $\begin{array}{l}27-02-07-05 \mathrm{~F} . \\
26\end{array}$ \\
\hline $\begin{array}{l}\text { Taking Indian } \\
\text { Timber }\end{array}$ & $3 \mathrm{rd}$ & Sears, Nathan & $5 / 1 / 1857$ & $\begin{array}{l}27-02-07-05 \mathrm{~F} . \\
25\end{array}$ \\
\hline $\begin{array}{l}\text { Taking Indian } \\
\text { Timber }\end{array}$ & $3 \mathrm{rd}$ & $\begin{array}{l}\text { Snodgrass, } \\
\text { Charles }\end{array}$ & $1 / 1 / 1858$ & $\begin{array}{l}27-02-07-05 \mathrm{~F} . \\
60\end{array}$ \\
\hline $\begin{array}{l}\text { Taking Indian } \\
\text { Timber }\end{array}$ & $3 \mathrm{rd}$ & Stafford, John & $11 / 1 / 1859$ & $\begin{array}{l}27-02-07-06 \mathrm{~F} . \\
01\end{array}$ \\
\hline $\begin{array}{l}\text { Taking Indian } \\
\text { Timber }\end{array}$ & $3 \mathrm{rd}$ & $\begin{array}{l}\text { Stephens, } \\
\text { William }\end{array}$ & $2 / 1 / 1859$ & $\begin{array}{l}27-02-07-06 \mathrm{~F} . \\
10\end{array}$ \\
\hline $\begin{array}{l}\text { Taking Indian } \\
\text { Timber }\end{array}$ & $3 \mathrm{rd}$ & $\begin{array}{l}\text { Throckmorton, } \\
\text { William }\end{array}$ & $8 / 1 / 1859$ & $\begin{array}{l}27-02-07-06 \mathrm{~F} . \\
57\end{array}$ \\
\hline $\begin{array}{l}\text { Taking Indian } \\
\text { Timber }\end{array}$ & $3 \mathrm{rd}$ & Watland, Henry & $1 / 1 / 1859$ & $\begin{array}{l}27-02-08-01 \mathrm{~F} . \\
13\end{array}$ \\
\hline $\begin{array}{l}\text { Taking Indian } \\
\text { Timber }\end{array}$ & $3 \mathrm{rd}$ & Weaver, W.M. & $2 / 25 / 1860$ & $\begin{array}{l}27-02-08-01 \mathrm{~F} . \\
21\end{array}$ \\
\hline $\begin{array}{l}\text { Taking Indian } \\
\text { Timber }\end{array}$ & $3 \mathrm{rd}$ & Wells, Abraham & $4 / 1 / 1859$ & $\begin{array}{l}27-02-08-01 \mathrm{~F} . \\
25\end{array}$ \\
\hline $\begin{array}{l}\text { Taking Indian } \\
\text { Timber }\end{array}$ & $3 \mathrm{rd}$ & Williams, Jesse & $4 / 30 / 1860$ & $\begin{array}{l}27-02-08-01 \mathrm{~F} . \\
51\end{array}$ \\
\hline $\begin{array}{l}\text { Taking Indian } \\
\text { Timber }\end{array}$ & $3 \mathrm{rd}$ & Wills, William & $5 / 1 / 1857$ & $\begin{array}{l}27-02-08-01 \mathrm{~F} . \\
60\end{array}$ \\
\hline
\end{tabular}




\begin{tabular}{|c|c|c|c|c|}
\hline $\begin{array}{l}\text { Taking Timber } \\
\text { from Indian } \\
\text { Lands }\end{array}$ & 3rd & Beck, Henry & $5 / 1860$ & $\begin{array}{l}27-02-05-06 \mathrm{~F} . \\
56\end{array}$ \\
\hline $\begin{array}{l}\text { Taking Timber } \\
\text { from Indian } \\
\text { Lands }\end{array}$ & 3rd & Dunbar, Henry & $11 / 28 / 1859$ & $\begin{array}{l}27-02-06-03 \mathrm{~F} . \\
51\end{array}$ \\
\hline $\begin{array}{l}\text { Taking Timber } \\
\text { from Indian } \\
\text { Lands }\end{array}$ & $3 \mathrm{rd}$ & Ewing, Milton & $11 / 1 / 1859$ & $\begin{array}{l}27-02-06-04 \mathrm{~F} . \\
14\end{array}$ \\
\hline $\begin{array}{l}\text { Taking Timber } \\
\text { from Indian } \\
\text { Lands }\end{array}$ & 3rd & Hulse, John & $6 / 1855$ & $\begin{array}{l}27-02-06-06 \mathrm{~F} . \\
31\end{array}$ \\
\hline $\begin{array}{l}\text { Taking Timber } \\
\text { from Indian } \\
\text { Lands }\end{array}$ & 3rd & Jeffries, William & $2 / 1 / 1860$ & $\begin{array}{l}27-02-06-06 \mathrm{~F} . \\
55\end{array}$ \\
\hline $\begin{array}{l}\text { Taking Timber } \\
\text { from Indian } \\
\text { Lands }\end{array}$ & 2nd & Jolly, James & $2 / 1 / 1860$ & $\begin{array}{l}27-02-06-07 \mathrm{~F} . \\
10\end{array}$ \\
\hline $\begin{array}{l}\text { Taking Timber } \\
\text { from Indian } \\
\text { Lands }\end{array}$ & 3 rd & Jones, James & $12 / 2 / 1859$ & $\begin{array}{l}27-02-06-07 \mathrm{~F} . \\
14\end{array}$ \\
\hline $\begin{array}{l}\text { Taking Timber } \\
\text { from Indian } \\
\text { Lands }\end{array}$ & 2nd & Kress, G.W. & $2 / 3 / 1860$ & $\begin{array}{l}27-02-06-07 \mathrm{~F} . \\
60\end{array}$ \\
\hline $\begin{array}{l}\text { Taking Timber } \\
\text { from Indian } \\
\text { Lands }\end{array}$ & 3rd & Lears, Charles & $12 / 2 / 1859$ & $\begin{array}{l}27-02-07-01 \mathrm{~F} . \\
14\end{array}$ \\
\hline $\begin{array}{l}\text { Taking Timber } \\
\text { from Indian } \\
\text { Lands }\end{array}$ & 3rd & Lears, Nathan & $12 / 2 / 1859$ & $\begin{array}{l}27-02-07-01 \mathrm{~F} . \\
14\end{array}$ \\
\hline $\begin{array}{l}\text { Taking Timber } \\
\text { from Indian } \\
\text { Lands }\end{array}$ & $\begin{array}{l}\text { Pottawatomie } \\
\text { Cnty. }\end{array}$ & Mullen, G.L & $2 / 15 / 1858$ & $\begin{array}{l}27-02-07-02 \mathrm{~F} . \\
52\end{array}$ \\
\hline $\begin{array}{l}\text { Taking Timber } \\
\text { from Indian } \\
\text { Lands }\end{array}$ & $3 \mathrm{rd}$ & Ruble, Jonathan & $2 / 1 / 1859$ & $\begin{array}{l}27-02-07-04 \mathrm{~F} . \\
44\end{array}$ \\
\hline $\begin{array}{l}\text { Taking Timber } \\
\text { from Indian } \\
\text { Lands }\end{array}$ & 3rd & Schaffer, Joseph & $1 / 1 / 1859$ & $\begin{array}{l}27-02-07-05 \mathrm{~F} . \\
13\end{array}$ \\
\hline Theft & 2nd & Alkiere & $9 / 1856$ & $\begin{array}{l}27-02-05-06 \mathrm{~F} . \\
09\end{array}$ \\
\hline $\begin{array}{l}\text { Theft, Horse } \\
\text { Stealing }\end{array}$ & $?$ & Babcock, C.W. & $10 / 8 / 1856$ & $\begin{array}{l}27-02-05-06 \mathrm{~F} . \\
22\end{array}$ \\
\hline Treason & 2nd & $\begin{array}{l}\text { Robinson, } \\
\text { Charles }\end{array}$ & $5 / 24 / 1856$ & $\begin{array}{l}27-02-07-04 \mathrm{~F} . \\
37\end{array}$ \\
\hline Treason & 2nd & $\begin{array}{l}\text { Robinson, } \\
\text { Charles \& Lane, } \\
\text { James \& Brown, } \\
\text { George \& Ward, } \\
\text { Samuel }\end{array}$ & $5 / 17 / 1856$ & $\begin{array}{l}27-02-07-04 \mathrm{~F} . \\
11\end{array}$ \\
\hline Trespass & 2nd & Baine, Clayton & $2 / 1 / 1858$ & $\begin{array}{l}27-02-05-06 \mathrm{~F} . \\
27\end{array}$ \\
\hline Trespass & 2nd & Copeland, John & $7 / 1 / 1858$ & $\begin{array}{l}27-02-06-02 \mathrm{~F} . \\
18\end{array}$ \\
\hline Trespass & 2nd & Lane, Gordon & $10 / 1 / 1858$ & $\begin{array}{l}27-02-07-01 \mathrm{~F} . \\
07\end{array}$ \\
\hline
\end{tabular}




\begin{tabular}{|c|c|c|c|c|}
\hline Trespass & 2nd & $\begin{array}{l}\text { Randolph, } \\
\text { Milton }\end{array}$ & $2 / 4 / 1858$ & $\begin{array}{l}\text { 27-02-07-04 F. } \\
03\end{array}$ \\
\hline Trespass & 2nd & $\begin{array}{l}\text { Roberts, James } \\
\text { \& McDonald, } \\
\text { Brant \& Sharp, } \\
\text { Benjamin }\end{array}$ & $10 / 1 / 1858$ & $\begin{array}{l}27-02-07-04 \mathrm{~F} . \\
33\end{array}$ \\
\hline $\begin{array}{l}\text { Trespass in } \\
\text { Indian Country }\end{array}$ & 2nd & Miller, Francis & $3 / 19 / 1860$ & $\begin{array}{l}27-02-07-02 \mathrm{~F} . \\
07\end{array}$ \\
\hline $\begin{array}{l}\text { Trespass in } \\
\text { Indian Country }\end{array}$ & Franklin Cnty. & Shoarg, Solomon & $3 / 17 / 1860$ & $\begin{array}{l}27-02-07-05 \mathrm{~F} . \\
44\end{array}$ \\
\hline $\begin{array}{l}\text { Trespass on } \\
\text { Indian Country }\end{array}$ & $1 \mathrm{st}$ & Milo, Claude & $2 / 25 / 1858$ & $\begin{array}{l}27-02-07-02 \mathrm{~F} . \\
20\end{array}$ \\
\hline $\begin{array}{l}\text { Trespass on } \\
\text { Indian Country }\end{array}$ & 2nd & Mings, John & $3 / 21 / 1860$ & $\begin{array}{l}27-02-07-02 \mathrm{~F} . \\
21\end{array}$ \\
\hline $\begin{array}{l}\text { Trespass on } \\
\text { Indian Lands }\end{array}$ & 2nd & $\begin{array}{l}\text { Allen, David \& } \\
\text { Adams, } \\
\text { Archibald }\end{array}$ & $10 / 1858$ & $\begin{array}{l}27-02-05-06 \mathrm{~F} . \\
11\end{array}$ \\
\hline $\begin{array}{l}\text { Trespass on } \\
\text { Indian Lands }\end{array}$ & 2nd & Carson, Jonah & $5 / 1860$ & $\begin{array}{l}27-02-06-01 \mathrm{~F} . \\
13\end{array}$ \\
\hline $\begin{array}{l}\text { Trespass on } \\
\text { Indian Lands }\end{array}$ & 2nd & Collins, William & $5 / 19 / 1860$ & $\begin{array}{l}27-02-06-02 \mathrm{~F} . \\
11\end{array}$ \\
\hline $\begin{array}{l}\text { Trespass on } \\
\text { Indian Lands }\end{array}$ & 2nd & Daniels, Eli & $2 / 20 / 1859$ & $\begin{array}{l}27-02-06-03 \mathrm{~F} . \\
02\end{array}$ \\
\hline $\begin{array}{l}\text { Trespass on } \\
\text { Indian Lands }\end{array}$ & 2nd & Keizer, William & $3 / 17 / 1860$ & $\begin{array}{l}27-02-06-07 \mathrm{~F} . \\
29\end{array}$ \\
\hline $\begin{array}{l}\text { Trespass on } \\
\text { Indian Lands }\end{array}$ & 2nd & $\begin{array}{l}\text { Leonard, Calvin } \\
\text { \& Hodges, } \\
\text { Joshua }\end{array}$ & $3 / 22 / 1860$ & $\begin{array}{l}27-02-07-01 \mathrm{~F} . \\
15\end{array}$ \\
\hline $\begin{array}{l}\text { Trespass on } \\
\text { Indian Lands }\end{array}$ & 2nd & Snow, R.P. & $11 / 1 / 1859$ & $\begin{array}{l}27-02-07-05 \mathrm{~F} . \\
61\end{array}$ \\
\hline $\begin{array}{l}\text { Trespass on } \\
\text { Indian Lands }\end{array}$ & 2nd & $\begin{array}{l}\text { Ward, Dennis \& } \\
\text { Kiezer, Daniel }\end{array}$ & $3 / 21 / 1860$ & $\begin{array}{l}27-02-08-01 \mathrm{~F} . \\
07\end{array}$ \\
\hline $\begin{array}{l}\text { Trespass on } \\
\text { Indian Lands }\end{array}$ & 3rd & Williams, Jesse & $2 / 1 / 1860$ & $\begin{array}{l}27-02-08-01 \mathrm{~F} . \\
53\end{array}$ \\
\hline $\begin{array}{l}\text { Trespass on } \\
\text { School Lands }\end{array}$ & 2nd & $\begin{array}{l}\text { Ackenburg, } \\
\text { William }\end{array}$ & $11 / 1859$ & $\begin{array}{l}27-02-05-06 \mathrm{~F} . \\
01\end{array}$ \\
\hline $\begin{array}{l}\text { Trespass on } \\
\text { School Lands }\end{array}$ & 2nd & Alder, Bird & $11 / 1859$ & $\begin{array}{l}27-02-05-06 \mathrm{~F} . \\
07\end{array}$ \\
\hline $\begin{array}{l}\text { Trespass on } \\
\text { School Lands }\end{array}$ & 2nd & Biths, Silas & $11 / 1 / 1859$ & $\begin{array}{l}27-02-05-07 \mathrm{~F} . \\
10\end{array}$ \\
\hline $\begin{array}{l}\text { Trespass on } \\
\text { School Lands }\end{array}$ & 2nd & Block, Thomas & $11 / 1 / 1859$ & $\begin{array}{l}27-02-05-07 \mathrm{~F} . \\
12\end{array}$ \\
\hline $\begin{array}{l}\text { Trespass on } \\
\text { School Lands }\end{array}$ & 2nd & Campbell, Neil & $11 / 1 / 1859$ & $\begin{array}{l}27-02-06-01 \mathrm{~F} . \\
29\end{array}$ \\
\hline $\begin{array}{l}\text { Trespass on } \\
\text { School Lands }\end{array}$ & 2nd & Craft, George & $10 / 1 / 1859$ & $\begin{array}{l}27-02-06-02 \mathrm{~F} . \\
26\end{array}$ \\
\hline $\begin{array}{l}\text { Trespass on } \\
\text { School Lands }\end{array}$ & 2nd & Daran, Edward & $11 / 1 / 1859$ & $\begin{array}{l}27-02-06-03 \mathrm{~F} . \\
03\end{array}$ \\
\hline $\begin{array}{l}\text { Trespass on } \\
\text { School Lands }\end{array}$ & 2nd & Duran, William & $11 / 1 / 1859$ & $\begin{array}{l}27-02-06-03 \mathrm{~F} . \\
04\end{array}$ \\
\hline $\begin{array}{l}\text { Trespass on } \\
\text { School Lands }\end{array}$ & 2nd & $\begin{array}{l}\text { Eikenberry, } \\
\text { William }\end{array}$ & $5 / 1860$ & $\begin{array}{l}27-02-06-04 \mathrm{~F} . \\
04\end{array}$ \\
\hline $\begin{array}{l}\text { Trespass on } \\
\text { School Lands }\end{array}$ & 2nd & Elliott, Isaiah & $11 / 1 / 1859$ & $\begin{array}{l}27-02-06-04 \mathrm{~F} . \\
06\end{array}$ \\
\hline $\begin{array}{l}\text { Trespass on } \\
\text { School Lands }\end{array}$ & 2nd & Fultz, Abraham & $3 / 1 / 1860$ & $\begin{array}{l}27-02-06-04 \mathrm{~F} . \\
63\end{array}$ \\
\hline
\end{tabular}




\begin{tabular}{|c|c|c|c|c|}
\hline $\begin{array}{l}\text { Trespass on } \\
\text { School Lands }\end{array}$ & 2nd & Fultz, Robert & $3 / 1 / 1860$ & $\begin{array}{l}27-02-06-04 \mathrm{~F} . \\
64\end{array}$ \\
\hline $\begin{array}{l}\text { Trespass on } \\
\text { School Lands }\end{array}$ & 2nd & Fultz, William & $3 / 1 / 1860$ & $\begin{array}{l}27-02-06-04 \mathrm{~F} . \\
65\end{array}$ \\
\hline $\begin{array}{l}\text { Trespass on } \\
\text { School Lands }\end{array}$ & 2 nd & Galeston, Milton & $11 / 1 / 1859$ & $\begin{array}{l}27-02-06-05 \mathrm{~F} . \\
04\end{array}$ \\
\hline $\begin{array}{l}\text { Trespass on } \\
\text { School Lands }\end{array}$ & 2nd & Galeston. John & $11 / 1 / 1859$ & $\begin{array}{l}27-02-06-05 \mathrm{~F} . \\
03\end{array}$ \\
\hline $\begin{array}{l}\text { Trespass on } \\
\text { School Lands }\end{array}$ & 2nd & Gilman, Victor & $2 / 1 / 1861$ & $\begin{array}{l}27-02-06-05 \mathrm{~F} . \\
20\end{array}$ \\
\hline $\begin{array}{l}\text { Trespass on } \\
\text { School Lands }\end{array}$ & 2nd & Haworth, Joel & $11 / 1 / 1859$ & $\begin{array}{l}27-02-06-05 \mathrm{~F} . \\
69\end{array}$ \\
\hline $\begin{array}{l}\text { Trespass on } \\
\text { School Lands }\end{array}$ & 3rd & Hopkins, George & $8 / 1858$ & $\begin{array}{l}27-02-06-06 \mathrm{~F} . \\
20\end{array}$ \\
\hline $\begin{array}{l}\text { Trespass on } \\
\text { School Lands }\end{array}$ & $3 \mathrm{rd}$ & Houston, John & $8 / 1 / 1858$ & $\begin{array}{l}27-02-06-06 \mathrm{~F} . \\
26\end{array}$ \\
\hline $\begin{array}{l}\text { Trespass on } \\
\text { School Lands }\end{array}$ & 2nd & Hutchinson, John & $11 / 1 / 1859$ & $\begin{array}{l}27-02-06-06 \mathrm{~F} . \\
43\end{array}$ \\
\hline $\begin{array}{l}\text { Trespass on } \\
\text { School Lands }\end{array}$ & 2nd & $\begin{array}{l}\text { Hutchinson, } \\
\text { Josiah }\end{array}$ & $11 / 1 / 1859$ & $\begin{array}{l}27-02-06-06 \mathrm{~F} . \\
44\end{array}$ \\
\hline $\begin{array}{l}\text { Trespass on } \\
\text { School Lands }\end{array}$ & 3 rd & Hutton, Basil & $9 / 11 / 1858$ & $\begin{array}{l}27-02-06-06 \mathrm{~F} . \\
45\end{array}$ \\
\hline $\begin{array}{l}\text { Trespass on } \\
\text { School Lands }\end{array}$ & 3rd & Hutton, Joseph & $9 / 11 / 1858$ & $\begin{array}{l}27-02-06-06 \mathrm{~F} . \\
46\end{array}$ \\
\hline $\begin{array}{l}\text { Trespass on } \\
\text { School Lands }\end{array}$ & 2 nd & Johnson, Ralph & $11 / 1 / 1859$ & $\begin{array}{l}27-02-06-07 \mathrm{~F} . \\
08\end{array}$ \\
\hline $\begin{array}{l}\text { Trespass on } \\
\text { School Lands }\end{array}$ & 3nd & Kelley, Allen & $11 / 1 / 1859$ & $\begin{array}{l}27-02-06-07 \mathrm{~F} . \\
30\end{array}$ \\
\hline $\begin{array}{l}\text { Trespass on } \\
\text { School Lands }\end{array}$ & 2nd & $\begin{array}{l}\text { McCormick, } \\
\text { Mathew }\end{array}$ & $11 / 1 / 1859$ & $\begin{array}{l}27-02-07-01 \mathrm{~F} . \\
61\end{array}$ \\
\hline $\begin{array}{l}\text { Trespass on } \\
\text { School Lands }\end{array}$ & 2nd & $\begin{array}{l}\text { McCullough, } \\
\text { Walter }\end{array}$ & $11 / 1 / 1859$ & $\begin{array}{l}27-02-07-01 \mathrm{~F} . \\
64\end{array}$ \\
\hline $\begin{array}{l}\text { Trespass on } \\
\text { School Lands }\end{array}$ & 2nd & $\begin{array}{l}\text { McMillian, } \\
\text { David }\end{array}$ & $11 / 1 / 1859$ & $\begin{array}{l}27-02-07-01 \mathrm{~F} . \\
87\end{array}$ \\
\hline $\begin{array}{l}\text { Trespass on } \\
\text { School Lands }\end{array}$ & 2nd & $\begin{array}{l}\text { Morgan, } \\
\text { Christopher }\end{array}$ & $4 / 1 / 1859$ & $\begin{array}{l}27-02-07-02 \mathrm{~F} . \\
32\end{array}$ \\
\hline $\begin{array}{l}\text { Trespass on } \\
\text { School Lands }\end{array}$ & 2nd & Morrison, James & $3 / 1 / 1859$ & $\begin{array}{l}27-02-07-02 \mathrm{~F} . \\
47\end{array}$ \\
\hline $\begin{array}{l}\text { Trespass on } \\
\text { School Lands }\end{array}$ & 2nd & Pitts, Silas & $5 / 1 / 1860$ & $\begin{array}{l}27-02-07-03 \mathrm{~F} . \\
65\end{array}$ \\
\hline $\begin{array}{l}\text { Trespass on } \\
\text { School Lands }\end{array}$ & 2nd & Pitts, Strand & $3 / 6 / 1860$ & $\begin{array}{l}27-02-07-03 \mathrm{~F} . \\
67\end{array}$ \\
\hline $\begin{array}{l}\text { Trespass on } \\
\text { School Lands }\end{array}$ & 2nd & Pitts, William & $11 / 1 / 1859$ & $\begin{array}{l}27-02-07-03 \mathrm{~F} . \\
68\end{array}$ \\
\hline $\begin{array}{l}\text { Trespass on } \\
\text { School Lands }\end{array}$ & 2nd & Plumb, Jacob & $11 / 1 / 1859$ & $\begin{array}{l}27-02-07-03 \mathrm{~F} . \\
69\end{array}$ \\
\hline $\begin{array}{l}\text { Trespass on } \\
\text { School Lands }\end{array}$ & 2nd & Plumb, Jacob & $5 / 1 / 1860$ & $\begin{array}{l}27-02-07-03 \mathrm{~F} . \\
70\end{array}$ \\
\hline $\begin{array}{l}\text { Trespass on } \\
\text { School Lands }\end{array}$ & $34 d$ & Pool, James & $4 / 7 / 1860$ & $\begin{array}{l}27-02-07-03 \mathrm{~F} . \\
71\end{array}$ \\
\hline $\begin{array}{l}\text { Usurpation of } \\
\text { Office }\end{array}$ & 2nd & Cohee, Vincent & $4 / 1 / 1856$ & $\begin{array}{l}27-02-06-02 \mathrm{~F} . \\
05\end{array}$ \\
\hline $\begin{array}{l}\text { Usurpation of } \\
\text { Office }\end{array}$ & 2nd & $\begin{array}{l}\text { Cunningham, } \\
\text { John }\end{array}$ & $4 / 1 / 1856$ & $\begin{array}{l}27-02-06-02 \mathrm{~F} . \\
40\end{array}$ \\
\hline $\begin{array}{l}\text { Usurpation of } \\
\text { Office }\end{array}$ & 2nd & Dunn, James & $1 / 8 / 1857$ & $\begin{array}{l}27-02-06-03 \mathrm{~F} . \\
55\end{array}$ \\
\hline
\end{tabular}




\begin{tabular}{|c|c|c|c|c|}
\hline $\begin{array}{l}\text { Usurpation of } \\
\text { Office }\end{array}$ & 2nd & $\begin{array}{l}\text { Font, John \& } \\
\text { Reader, Samuel }\end{array}$ & $1 / 15 / 1856$ & $\begin{array}{l}27-02-06-04 \mathrm{~F} . \\
40\end{array}$ \\
\hline $\begin{array}{l}\text { Usurpation of } \\
\text { Office }\end{array}$ & 2nd & Foster, Freeman & $3 / 19 / 1855$ & $\begin{array}{l}27-02-06-04 \mathrm{~F} . \\
45\end{array}$ \\
\hline $\begin{array}{l}\text { Usurpation of } \\
\text { Office }\end{array}$ & ? & $\begin{array}{l}\text { Harris, } \\
\text { Pennington }\end{array}$ & $10 / 9 / 1855$ & $\begin{array}{l}27-02-06-05 \mathrm{~F} . \\
57\end{array}$ \\
\hline $\begin{array}{l}\text { Usurpation of } \\
\text { Office }\end{array}$ & 1st, 2nd & $\begin{array}{l}\text { Robinson, } \\
\text { Charles }\end{array}$ & $4 / 23 / 1856$ & $\begin{array}{l}27-02-07-04 \mathrm{~F} . \\
36\end{array}$ \\
\hline $\begin{array}{l}\text { Usurpation of } \\
\text { Office }\end{array}$ & 2nd & Shimmons, J.H. & $1 / 15 / 1856$ & $\begin{array}{l}27-02-07-05 \mathrm{~F} . \\
42\end{array}$ \\
\hline $\begin{array}{l}\text { Usurpation of } \\
\text { Office }\end{array}$ & $1 \mathrm{st}$ & Wimp, William & $3 / 1 / 1859$ & $\begin{array}{l}27-02-08-01 \mathrm{~F} . \\
70\end{array}$ \\
\hline $\begin{array}{l}\text { Various Crimes } \\
\text { and } \\
\text { Misdemeanors }\end{array}$ & $?$ & Cleveland, L.G & $9 / 15 / 1856$ & $\begin{array}{l}27-02-06-01 \mathrm{~F} . \\
43\end{array}$ \\
\hline $\begin{array}{l}\text { Writ of Habeas } \\
\text { Corpus }\end{array}$ & $?$ & Deadorf, Joel & $2 / 13 / 1857$ & $\begin{array}{l}27-02-06-03 \mathrm{~F} . \\
21\end{array}$ \\
\hline$?$ & Douglas Cnty. & $?$ & $9 / 20 / 1856$ & $\begin{array}{l}27-02-07-07 \mathrm{~F} . \\
20\end{array}$ \\
\hline$?$ & ? & $\begin{array}{l}\text { Brohn, John \& } \\
\text { Cline, James \& } \\
\text { King, Nelson }\end{array}$ & $10 / 1856$ & $\begin{array}{l}\text { 27-02-05-07 F. } \\
40\end{array}$ \\
\hline$?$ & $1 \mathrm{st}$ & Clark, John & $10 / 10 / 1856$ & $\begin{array}{l}27-02-06-01 \mathrm{~F} . \\
36\end{array}$ \\
\hline$?$ & $3 \mathrm{rd}$ & $\begin{array}{l}\text { Crittenden, } \\
\text { Lieutenant }\end{array}$ & $?$ & $\begin{array}{l}27-02-06-02 \mathrm{~F} . \\
33\end{array}$ \\
\hline$?$ & $?$ & Davis, Elioni & $8 / 13 / 1859$ & $\begin{array}{l}27-02-06-03 \mathrm{~F} . \\
10\end{array}$ \\
\hline$?$ & 2nd & Doyle, Daniel & $6 / 28 / 1859$ & $\begin{array}{l}27-02-06-03 \mathrm{~F} . \\
44\end{array}$ \\
\hline$?$ & 2nd & Garrett, Wesley & $6 / 28 / 1859$ & $\begin{array}{l}27-02-06-05 \mathrm{~F} . \\
10\end{array}$ \\
\hline$?$ & 2nd & Kilbourn, Henry & $1 / 9 / 1857$ & $\begin{array}{l}27-02-06-07 \mathrm{~F} . \\
49\end{array}$ \\
\hline$?$ & $?$ & Mitchel & $10 / 14 / 1856$ & $\begin{array}{l}27-02-07-02 \mathrm{~F} . \\
22\end{array}$ \\
\hline$?$ & $1 \mathrm{st}$ & $\begin{array}{l}\text { Newcomb, } \\
\text { Artemus }\end{array}$ & $9 / 12 / 1859$ & $\begin{array}{l}27-02-07-03 \mathrm{~F} . \\
01\end{array}$ \\
\hline$?$ & 2nd & Nichols, R.B. & $11 / 24 / 1856$ & $\begin{array}{l}27-02-07-03 \mathrm{~F} . \\
06\end{array}$ \\
\hline$?$ & 2nd & Ott, William & $6 / 28 / 1859$ & $\begin{array}{l}27-02-07-03 \mathrm{~F} . \\
18\end{array}$ \\
\hline ? & $1 \mathrm{st}$ & Paine, Alfred & $10 / 21 / 1856$ & $\begin{array}{l}27-02-07-03 \mathrm{~F} . \\
22\end{array}$ \\
\hline ? & ? & Paul, Samuel & $12 / 27 / 1856$ & $\begin{array}{l}27-02-07-03 \mathrm{~F} . \\
41\end{array}$ \\
\hline$?$ & 2nd & Preston, Charles & $11 / 18 / 1856$ & $\begin{array}{l}27-02-07-03 \mathrm{~F} . \\
76\end{array}$ \\
\hline$?$ & $3 \mathrm{rd}$ & Rochefus, Fred & $4 / 19 / 1860$ & $\begin{array}{l}27-02-07-04 \mathrm{~F} . \\
45\end{array}$ \\
\hline$?$ & $3 \mathrm{rd}$ & Schell, Alfred & $11 / 1 / 1859$ & $\begin{array}{l}27-02-07-05 \mathrm{~F} . \\
14\end{array}$ \\
\hline$?$ & $3 \mathrm{rd}$ & Smith, James & $6 / 12 / 1859$ & $\begin{array}{l}27-02-07-05 \mathrm{~F} . \\
55\end{array}$ \\
\hline$?$ & 3rd & Teonsley, James & $9 / 8 / 1860$ & $\begin{array}{l}27-02-07-06 \mathrm{~F} . \\
43\end{array}$ \\
\hline
\end{tabular}




\begin{tabular}{|l|l|l|l|l|}
\hline$?$ & 2nd & $\begin{array}{l}\text { Updegraft, } \\
\text { Derrick }\end{array}$ & $11 / 25 / 1856$ & $\begin{array}{l}27-02-07-07 \mathrm{~F} . \\
22\end{array}$ \\
\hline$?$ & 2nd & Updegraft, & $11 / 29 / 1856$ & $\begin{array}{l}27-02-07-07 \mathrm{~F} . \\
24\end{array}$ \\
\hline$?$ & & William & & $\begin{array}{l}27-02-08-01 \mathrm{~F} . \\
84\end{array}$ \\
\hline
\end{tabular}

